\title{
THE LAST FORCING STANDING WITH DIAMONDS
}

\author{
ANDRZEJ ROSŁANOWSKI AND SAHARON SHELAH
}

\begin{abstract}
This article continues Rosłanowski and Shelah 14, 19. We introduce a new property of $(<\lambda)$-strategically complete forcing notions which implies that their $\lambda$-support iterations do not collapse $\lambda^{+}$.
\end{abstract}

\section{INTRODUCTION}

While there are still a lot of open problems left in the theory of forcing iterated with finite and/or countable supports and we still need to expand our preservation theorems, there is a sense that we understand these iterations pretty well. Therefore it is natural to look at iterations with uncountable supports and ask for parallel theorems. The first attempt could be to do nothing special and just repeat what has been done for CS iterations. We could start in the way suggested already in Shelah [20] (but not used there).

Definition 1.1. Let $\lambda=\lambda^{<\lambda}$. A notion of forcing $\mathbb{P}$ is said to be $\lambda$-proper in the standard sense (or just: $\lambda$-proper) if for all sufficiently large regular cardinals $\chi$, there is some $x \in \mathcal{H}(\chi)$ such that whenever $M$ is an elementary submodel of $\mathcal{H}(\chi)$ satisfying

$$
|M|=\lambda, \quad \mathbb{P}, x \in M \quad M^{<\lambda} \subseteq M
$$

and $p$ is an element of $M \cap \mathbb{P}$, then there is a condition $q \geq p$ such that

$$
q \Vdash \text { " } M\left[G_{\mathbb{P}}\right] \cap \operatorname{Ord}=M \cap \operatorname{Ord} " .
$$

The $\lambda$-properness may seem to be a straightforward generalization of "proper", with the right consequences in place. For instance:

Theorem 1.2 (Folklore; cf. Hyttinen and Rautila [8, Section 3]). Assume $\lambda^{<\lambda}=\lambda$ is an uncountable cardinal.

(1) If a forcing notion $\mathbb{P}$ is either strategically $(\leq \lambda)$-complete or it satisfies the $\lambda^{+}$-chain condition, then $\mathbb{P}$ is $\lambda$-proper.

(2) If $\mathbb{P}$ is $\lambda$-proper, $p \in \mathbb{P}, \underset{\sim}{A}$ is a $\mathbb{P}$-name for a set of ordinals and $p \Vdash|A| \leq \lambda$, then there are a condition $q \in \mathbb{P}$ stronger than $p$ and a set $B$ of size $\lambda$ such that $q \Vdash \underset{\sim}{A} \subseteq B$.

(3) If $\mathbb{P}$ is $\lambda$-proper, then

$$
\Vdash_{\mathbb{P}} \text { “ }\left(\lambda^{+}\right) \mathbf{V} \text { is a regular cardinal ". }
$$

Moreover, if $\mathbb{P}$ is also strategically $(<\lambda)$-complete, then the forcing with $\mathbb{P}$ preserves stationary subsets of $\lambda^{+}$.

Date: May 2017.

1991 Mathematics Subject Classification. Primary 03E40; Secondary: 03 E35.

Key words and phrases. Forcing, uncountable support iterations, not collapsing cardinals, proper forcing notions. 
Also chain condition results look similarly:

Theorem 1.3 (Folklore; cf. Eisworth [3, Proposition 3.1] and Abraham [1, Theorems 2.10, 2.12]). Assume $\lambda^{<\lambda}=\lambda, 2^{\lambda}=\lambda^{+}$, and let $\overline{\mathbb{P}}=\left\langle\mathbb{P}_{i}, \mathbb{Q}_{i}: i<\lambda^{++}\right\rangle$be a $\lambda$-support iteration such that

(a) $\mathbb{P}_{i}$ is $\lambda$-proper for $i \leq \lambda^{++}$

(b) $\Vdash_{\mathbb{P}_{i}} "\left|\mathbb{Q}_{i}\right| \leq \lambda^{+} "$.

Then

(1) $\mathbb{P}_{\lambda^{++}}$satisfies the $\lambda^{++}$-chain condition, and

(2) for each $i<\lambda^{++}$we have $\Vdash_{\mathbb{P}_{i}} 2^{\lambda}=\lambda^{+}$.

Proof. Abraham [1, Theorem 2.10, Theorem 2.12] gives the full proof of the theorem for CS iterations. Eisworth [3, Proposition 3.1] presents careful justification of 1.3(1). Let us sketch arguments justifying 1.3(2)

To argue that $\Vdash_{\mathbb{P}_{i}}{ } 2^{\lambda}=\lambda^{+}$" suppose towards contradiction that $p \in \mathbb{P}_{i}$, $i<\lambda^{++}$, and $p \Vdash_{\mathbb{P}_{i}}$ " $\underset{\sim}{x}: \lambda^{++} \longrightarrow 2^{\lambda}$ is one-to-one". For each $\xi<\lambda^{++}$fix a model $M_{\xi} \prec \mathcal{H}(\chi)$ such that

$$
\xi, \overline{\mathbb{P}}, i, p, x \in M_{\xi}, \quad\left|M_{\xi}\right|=\lambda \quad \text { and } \quad<\lambda M_{\xi} \subseteq M_{\xi} .
$$

Applying "a cleaning procedure" as in the proof of [3. Proposition 3.1] we may choose a set $I \subseteq \lambda^{++}$of size $\lambda^{++}$, a set $H \subseteq \lambda^{++}$and mappings $\pi_{\xi, \zeta}$ (for $\xi, \zeta \in I$ satisfying $\xi<\zeta)$ such that:

(a) $\pi_{\xi, \zeta}: M_{\xi} \stackrel{\text { onto }}{\longrightarrow} M_{\zeta}$ is an $\in$-isomorphism, $\pi_{\xi, \zeta}(\xi)=\zeta, \pi_{\xi, \zeta}(\underset{\sim}{x})=\underset{\sim}{x}, \pi_{\xi, \zeta}(p)=$ $p$

(b) $M_{\xi} \cap M_{\zeta} \cap \lambda^{++}=H$,

(c) $H \subseteq \min \left(M_{\xi} \cap \lambda^{++} \backslash H\right)$ and $M_{\xi} \cap \lambda^{++} \subseteq \min \left(M_{\zeta} \cap \lambda^{++} \backslash H\right)$, and

(d) $i \in H, \pi_{\xi, \zeta}\left\lceil H=\mathrm{id}_{H}, \lambda \subseteq H\right.$.

The following claim will immediately complete the proof.

Claim 1.3.1. If $r \in \mathbb{P}_{i}$ is $\left(M_{\xi}, \mathbb{P}_{i}\right)$-generic and $r \geq p$ and $\xi<\zeta$ are from $I$, then $r \Vdash_{\mathbb{P}_{i}} \underset{\sim}{x}(\xi)=\underset{\sim}{x}(\zeta)$.

Proof of the Claim. Suppose towards contradiction that $r^{*} \geq r, r^{*} \Vdash \underset{\sim}{x}(\xi) \neq \underset{\sim}{x}(\zeta)$. Take $r^{+} \geq r^{*}$ and $\ell<2, \alpha<\lambda$ such that

$$
r^{+} \Vdash “ \underset{\sim}{x}(\xi)(\alpha)=\ell \text { and } \underset{\sim}{x}(\zeta)(\alpha)=1-\ell " .
$$

The condition $r^{+}$is $\left(M_{\xi}, \mathbb{P}_{i}\right)$-generic, so there is $s \in M_{\xi} \cap \mathbb{P}_{i}$ such that $s \Vdash_{\mathbb{P}_{i}}$ $x(\xi)(\alpha)=\ell$ and $r^{+}, s$ are compatible, say $q \geq r^{+}, s$. Then $q$ is $\left(M_{\xi}, \mathbb{P}_{i}\right)$-generic so by [3], Claim 3.4] we also have $q \geq \pi_{\xi, \zeta}(s)$. But $\pi_{\xi, \zeta}(s) \Vdash \pi_{\xi, \zeta}(x)\left(\pi_{\xi, \zeta}(\xi)\right)\left(\pi_{\xi, \zeta}(\alpha)\right)=\ell$, so $\pi_{\xi, \zeta}(s) \Vdash \underset{\sim}{x}(\zeta)(\alpha)=\ell$. Hence $q \Vdash \underset{\sim}{x}(\xi)(\alpha)=\underset{\sim}{x}(\zeta)(\alpha)$, contradicting the choice of $r^{+}$and $q \geq r^{+}$.

What is missing? The main point of properness is the preservation theorem for CS iterations. If one tries to repeat the proof of the preservation theorem for $\lambda$-support iterations of $\lambda$-proper forcing notions, then one faces difficulties at limit stages of cofinality fewer than $\lambda$ caused by the fact that it is inconvenient to diagonalize $\lambda$ objects in less than $\lambda$ steps. This is a more serious obstacle than just a technicality. Let us consider the following forcing notion. 
Example 1.4 (Shelah [21, Appendix]). Assume that $\lambda=\lambda^{<\lambda}$ is an uncountable cardinal and let $\mathcal{S}_{\lambda}^{\lambda^{+}} \stackrel{\text { def }}{=}\left\{\delta<\lambda^{+}: \operatorname{cf}(\delta)=\lambda\right\}$. Suppose that a sequence $\left\langle A_{\delta}, h_{\delta}\right.$ : $\left.\delta \in \mathcal{S}_{\lambda}^{\lambda^{+}}\right\rangle$is such that for each $\delta \in \mathcal{S}_{\lambda}^{\lambda^{+}}$:

(a) $A_{\delta} \subseteq \delta$ otp $\left(A_{\delta}\right)=\lambda$ and $A_{\delta}$ is a club of $\delta$, and

(b) $h_{\delta}: A_{\delta} \longrightarrow 2$

The forcing notion $\mathbb{Q}^{*}=\mathbb{Q}^{*}\left(\left\langle A_{\delta}, h_{\delta}: \delta \in \mathcal{S}_{\lambda}^{\lambda^{+}}\right\rangle\right)$is defined as follows:

a condition in $\mathbb{Q}^{*}$ is a tuple $p=\left(u^{p}, v^{p}, \bar{e}^{p}, h^{p}\right)$ such that

(a) $u^{p} \in\left[\lambda^{+}\right]^{<\lambda}, v^{p} \subseteq \mathcal{S}_{\lambda}^{\lambda^{+}} \cap u^{p}$,

(b) $\bar{e}^{p}=\left\langle e_{\delta}^{p}: \delta \in v^{p}\right\rangle$, where each $e_{\delta}^{p}$ is a closed bounded non-empty subset of $A_{\delta}$, and $e_{\delta}^{p} \subseteq u^{p}$, and

(c) if $\delta \in v^{p}$, then $\max \left(e_{\delta}^{p}\right)=\sup \left(u^{p} \cap \delta\right)>\sup \left(v^{p} \cap \delta\right)$,

(d) $h^{p}: u^{p} \longrightarrow 2$ is such that for each $\delta \in v^{p}$ we have that $h^{p}\left\lceil e_{\delta} \subseteq h_{\delta}\right.$.

The order $\leq$ of $\mathbb{Q}^{*}$ is such that $p \leq q$ if and only if $u^{p} \subseteq u^{q}, h^{p} \subseteq h^{q}, v^{p} \subseteq v^{q}$, and for each $\delta \in v^{p}$ the set $e_{\delta}^{q}$ is an end-extension of $e_{\delta}^{p}$.

Plainly, under the assumptions of 1.4 the following holds true.

Observation 1.5. The forcing notion $\mathbb{Q}^{*}$ is $(<\lambda)$-complete and $\left|\mathbb{Q}^{*}\right|=\lambda^{+}$. It satisfies the $\lambda^{+}$-chain condition, so it is also $\lambda$-proper.

If $\lambda=\lambda^{<\lambda}$ is not inaccessible, $2^{\lambda^{+}}=\lambda^{++}$and $2^{\lambda}=\lambda^{+}$, then some $\lambda$-support iterations of forcing notions like $\mathbb{Q}^{*}$ are not $\lambda$-proper, as a matter of fact this bad effect happens quite often. The problem comes from the fact that if $\lambda$-support iterations of forcings of type $\mathbb{Q}^{*}$ were $\lambda$-proper, we could use Theorem 1.3 and a suitable bookkeeping device to build a forcing notion which forces " $\lambda=\lambda^{<\lambda}$ is not inaccessible and uniformization for continuous ladder systems holds true". However, this is not possible:

Theorem 1.6 (Shelah 21], 22, Appendix, Theorem 3.6(2)]). Assume $\theta<\lambda=$ $\operatorname{cf}(\lambda), 2^{\theta}=2^{<\lambda}=\lambda$. Furthermore suppose that for each $\delta \in \mathcal{S}_{\lambda}^{\lambda^{+}}$we have a club $A_{\delta}$ of $\delta$. Then we can find a sequence $\left\langle d_{\delta}: \delta \in \mathcal{S}_{\lambda}^{\lambda^{+}}\right\rangle$of colorings such that

- $d_{\delta}: A_{\delta} \longrightarrow 2$ and

- for any $h: \lambda^{+} \longrightarrow\{0,1\}$ for stationarily many $\delta \in \mathcal{S}_{\lambda}^{\lambda^{+}}$, the set $\left\{i \in A_{\delta}\right.$ : $\left.d_{\delta}(i) \neq h(i)\right\}$ is stationary in $A_{\delta}$.

Many positive results concerning not collapsing cardinals in iterations with uncountable supports are presented in literature already. For instance, Kanamori [10] considered iterations of $\lambda$-Sacks forcing notion (similar to the forcing $\mathbb{Q}^{2, \bar{E}}$; see Definition 2.18 and Remark 2.19) and he proved that under some circumstances these iterations preserve $\lambda^{+}$. Several conditions ensuring that $\lambda^{+}$is not collapsed in $\lambda$-support iterations were introduced in Shelah 23, 24. Fusion properties of iterations of tree-like forcing notions were used in Friedman and Zdomskyy [5] and Friedman, Honzik and Zdomskyy $\underline{4}$.

Numerous strong versions of $\lambda$-properness were studied by the authors in a series of articles Rosłanowski and Shelah [14, 15, 16, 17, 18, 19. Each of those conditions was meant to be applicable to some natural forcing notions adding a new member of ${ }^{\lambda} \lambda$ without adding new elements of ${ }^{<\lambda} \lambda$. In some sense, they explained why the relevant forcings can be iterated (without collapsing cardinals). 
Also Eisworth [3] gave another (simpler) relative of the preservation theorem of 14.

The properties introduced in 14 and subsequent works we typically applicable to either very bounding forcing notions or forcings with trees with splittings on a stationary co-stationary set of levels. While those properties were quite general, we had problems to include the forcing notion $\mathbb{Q}_{\lambda}^{2}$ (see 2.18 below) in that framework. In 19 we managed to formulate a suitable property and show a relevant iteration theorem covering the forcing notions we were interested in (including $\mathbb{Q}_{\lambda}^{2}$ ) in the case when $\lambda$ is strongly inaccessible. Here we present a framework covering those forcings, including our "last forcing standing", under a very mild demand on $\lambda$ : it admits diamonds. In a sense, we generalize (and correct, see Remark 3.7) the property considered in [14]. Our property, the purely sequential properness over semi diamonds, is also close to the fuzzy properness over quasi-diamonds of [16, Definition A.3.6].

Notation and Terminology. Our notation is rather standard and compatible with that of classical textbooks (like Jech [9]). However, in forcing we keep the convention that a stronger condition is the larger one.

(1) Ordinal numbers will be denoted be the lower case initial letters of the Greek alphabet $\alpha, \beta, \gamma, \delta, \varepsilon$ and $\zeta$, and also by $\xi, i, j$ (with possible sub- and superscripts).

Cardinal numbers will be called $\kappa, \lambda ; \lambda$ will be always assumed to be a regular uncountable cardinal such that $\lambda^{<\lambda}=\lambda$.

Also, $\chi$ will denote a sufficiently large regular cardinal; $\mathcal{H}(\chi)$ is the family of all sets hereditarily of size less than $\chi$. Moreover, we fix a well ordering $<_{\chi}^{*}$ of $\mathcal{H}(\chi)$.

(2) We will consider several games of two players. One player will be called Generic or Complete, and we will refer to this player as "she". Her opponent will be called Antigeneric or Incomplete and will be referred to as "he".

(3) For a forcing notion $\mathbb{P}$, all $\mathbb{P}$-names for objects in the extension via $\mathbb{P}$ will be denoted with a tilde below (e.g., $\mathcal{\sim}, \underset{\sim}{X}$ ), and $G_{\mathbb{P}}$ will stand for the canonical $\mathbb{P}$-name for the generic filter in $\mathbb{P}$. The weakest element of $\mathbb{P}$ will be denoted by $\emptyset_{\mathbb{P}}$ (and we will always assume that there is one, and that there is no other condition equivalent to it).

By " $\lambda$-support iterations" we mean iterations in which domains of conditions are of size $\leq \lambda$. However, we will pretend that conditions in a $\lambda$-support iteration $\overline{\mathbb{Q}}=\left\langle\mathbb{P}_{\zeta}, \mathbb{Q}_{\zeta}: \zeta<\zeta^{*}\right\rangle$ are total functions on $\zeta^{*}$ and for $p \in \lim (\overline{\mathbb{Q}})$ and $\alpha \in \zeta^{*} \backslash \operatorname{dom}(\tilde{p})$ we will stipulate $p(\alpha)=\emptyset_{\mathbb{Q}_{\alpha}}$.

(4) A filter on $\lambda$ is a non-empty family of subsets of $\lambda$ closed under supersets and intersections and not containing $\emptyset$. A filter is $(<\kappa)$-complete if it is closed under intersections of $<\kappa$ members. (Note: we do allow principal filters or even $\{\lambda\}$.)

For a filter $D$ on $\lambda$, the family of all $D$-positive subsets of $\lambda$ is called $D^{+}$. (So $A \in D^{+}$if and only if $A \subseteq \lambda$ and $A \cap B \neq \emptyset$ for all $B \in D$.) By a normal filter on $\lambda$ we mean proper uniform filter closed under diagonal intersections.

\footnotetext{
${ }^{1}$ This should explain the title of this paper.
} 
(5) By a sequence we mean a function whose domain is a set of ordinals. For two sequences $\eta, \nu$ we write $\nu \triangleleft \eta$ whenever $\nu$ is a proper initial segment of $\eta$, and $\nu \unlhd \eta$ when either $\nu \triangleleft \eta$ or $\nu=\eta$. The length of a sequence $\eta$ is the order type of its domain and it is denoted by $\operatorname{lh}(\eta)$.

The set of all sequences with domain $\alpha$ and with values in $A$ is denoted by ${ }^{\alpha} A$ and we set ${ }^{<\alpha} A=\bigcup_{\beta<\alpha}{ }^{\beta} A$.

(6) A tree is a $\triangleleft$-downward closed set of sequences. A complete $\lambda$-tree is a tree $T \subseteq{ }^{<\lambda} \lambda$ such that every $\triangleleft$-chain of size less than $\lambda$ has a $\triangleleft$-bound in $T$ and for each $\eta \in T$ there is $\nu \in T$ such that $\eta \triangleleft \nu$.

Let $T \subseteq{ }^{<\lambda} \lambda$ be a tree. For $\eta \in T$ we let

$\operatorname{succ}_{T}(\eta)=\{\alpha<\lambda: \eta \curlyvee\langle\alpha\rangle \in T\} \quad$ and $\quad(T)_{\eta}=\{\nu \in T: \nu \triangleleft \eta$ or $\eta \unlhd \nu\}$.

We also let $\operatorname{root}(T)$ be the shortest $\eta \in T$ such that $\left|\operatorname{succ}_{T}(\eta)\right|>1$ and $\lim _{\lambda}(T)=\left\{\eta \in{ }^{\lambda} \lambda:(\forall \alpha<\lambda)(\eta\lceil\alpha \in T)\}\right.$.

\section{Preliminaries}

\subsection{Iterations of strategically complete forcing notions.}

Definition 2.1. Let $\mathbb{P}$ be a forcing notion.

(1) For an ordinal $\alpha$, let $\partial_{0}^{\alpha}(\mathbb{P})$ be the following game of two players, Complete and Incomplete:

the game lasts at most $\alpha$ moves and during a play the players attempt to construct a sequence $\left\langle\left(p_{i}, q_{i}\right): i<\alpha\right\rangle$ of pairs of conditions from $\mathbb{P}$ in such a way that

$$
(\forall j<i<\alpha)\left(p_{j} \leq q_{j} \leq p_{i}\right)
$$

and at the stage $i<\alpha$ of the game, first Incomplete chooses $p_{i}$ and then Complete chooses $q_{i}$.

Complete wins if and only if for every $i<\alpha$ there are legal moves for both players.

(2) A winning strategy $\mathbf{s t}^{0}$ of Complete in $\partial_{0}^{\alpha}(\mathbb{P})$ is regular if it instructs Complete to play $\emptyset_{\mathbb{P}}$ as long as Incomplete plays $\emptyset_{\mathbb{P}}$.

(3) The forcing notion $\mathbb{P}$ is strategically $(<\lambda)$-complete (strategically $(\leq \lambda)$ complete, respectively) if Complete has a winning strategy in the game $\partial_{0}^{\lambda}(\mathbb{P})\left(\partial_{0}^{\lambda+1}(\mathbb{P})\right.$, respectively).

Note that then Complete has also a regular winning strategy.

(4) Let a model $N \prec\left(\mathcal{H}(\chi), \in,<_{\chi}^{*}\right)$ be such that ${ }^{<\lambda} N \subseteq N,|N|=\lambda$ and $\mathbb{P} \in N$. We say that a condition $p \in \mathbb{P}$ is $(N, \mathbb{P})$-generic in the standard sense (or just: $(N, \mathbb{P})$-generic) if for every $\mathbb{P}$-name $\tau \in N$ for an ordinal we have $p \Vdash$ " $\tau \in N$ ".

For the rest of this subsection we assume that:

- $\overline{\mathbb{Q}}=\left\langle\mathbb{P}_{\zeta}, \mathbb{Q}_{\zeta}: \zeta\left\langle\zeta^{*}\right\rangle\right.$ is a $\lambda$-support iteration of strategically $(<\lambda)$ complete forcing notions, and

- for $\xi<\zeta^{*}, \mathbf{s t}_{\xi}^{0}$ is the $<_{\chi}^{*}$-first $\mathbb{P}_{\xi}$-name for a regular winning strategy of Complete in $\partial_{0}^{\lambda}\left(\mathbb{Q}_{\xi}\right)$, and

- a model $N \prec\left(\mathcal{H}(\chi), \in,<_{\chi}^{*}\right)$ is such that $|N|=\lambda,{ }^{<\lambda} N \subseteq N$ and $\overline{\mathbb{Q}}, \ldots \in N$.

Observation 2.2. $\mathbb{P}_{\zeta^{*}}$ is strategically $(<\lambda)$-complete. 
Observation 2.3. Suppose that $\zeta \in\left(\zeta^{*}+1\right) \cap N,\left\langle q_{\alpha}: \alpha<\delta\right\rangle \subseteq N \cap \mathbb{P}_{\zeta}, \delta<\lambda$, $r \in \mathbb{P}_{\zeta}$ is $\left(N, \mathbb{P}_{\zeta}\right)$-generic and $q_{\beta} \leq r$ for all $\beta<\delta$. Then there are conditions $q \in N \cap \mathbb{P}_{\zeta}$ and $r^{+} \in \mathbb{P}_{\zeta}$ such that $q \leq r^{+}, r \leq r^{+}$and $(\forall \beta<\delta)\left(q_{\beta} \leq q\right)$.

Lemma 2.4. Suppose that $\zeta \in\left(\zeta^{*}+1\right) \cap N$ is a limit ordinal of cofinality $\operatorname{cf}(\zeta)<\lambda$ and $r \in \mathbb{P}_{\zeta}$ is such that

$$
(\forall \varepsilon \in \zeta \cap N)\left(r\left\lceil\varepsilon \text { is }\left(N, \mathbb{P}_{\varepsilon}\right) \text {-generic }\right) .\right.
$$

Assume that conditions $q_{\alpha} \in N \cap \mathbb{P}_{\zeta}$ (for $\alpha<\delta, \delta<\lambda$ ) satisfy

(a) if $\alpha<\beta<\delta$, then $q_{\alpha} \leq q_{\beta} \leq r$, and

(b) if $\varepsilon \in N \cap \zeta, s \in \mathbb{P}_{\varepsilon}$ and $(\forall \alpha<\delta)\left(q_{\alpha}\lceil\varepsilon \leq s)\right.$, then

$$
s \Vdash_{\mathbb{P}_{\varepsilon}} \text { " the sequence }\left\langle q_{\alpha}(\varepsilon): \alpha<\delta\right\rangle \text { has an upper bound in } \mathbb{Q}_{\varepsilon} \text { ". }
$$

Then there are conditions $q \in N \cap \mathbb{P}_{\zeta}$ and $r^{+} \in \mathbb{P}_{\zeta}$ such that $q \leq r^{+}, r \leq r^{+}$and $(\forall \beta<\delta)\left(q_{\beta} \leq q\right)$.

Proof. The arguments here are similar to [14, Proposition 3.3], except that our iterands are strategically $(<\lambda)$-complete only (and not necessarily $(<\lambda)$-complete). This introduces some additional complexity.

Let $\left\langle i_{\gamma}: \gamma\langle\operatorname{cf}(\zeta)\rangle \subseteq N \cap \zeta\right.$ be a strictly increasing continuous sequence cofinal in $\zeta$ with $i_{0}=0$. By induction on $\gamma<\operatorname{cf}(\zeta)$ we choose $r_{\gamma}^{-}, r_{\gamma}$ and $r_{\gamma}^{*}$ such that

(i) $r_{\gamma}^{-} \in \mathbb{P}_{i_{\gamma}} \cap N$ is above (in $\mathbb{P}_{i_{\gamma}}$ ) of all $q_{\beta} \backslash i_{\gamma}$ for $\beta<\delta$;

(ii) $r_{\gamma}, r_{\gamma}^{*} \in \mathbb{P}_{i_{\gamma}}, r_{\gamma}^{-} \leq r_{\gamma} \leq r_{\gamma}^{*}$ and $r \nmid i_{\gamma} \leq r_{\gamma}$;

(iii) if $\gamma<\varepsilon<\operatorname{cf}(\zeta)$, then $r_{\gamma}^{-} \leq r_{\varepsilon}^{-}, r_{\gamma}^{*} \leq r_{\varepsilon}$, and

(iv) for each $\varepsilon<\zeta$ and $\gamma<\operatorname{cf}(\zeta)$ we have

$r_{\gamma}^{*}\left\lceil\varepsilon \Vdash \quad\right.$ " $\left\langle r_{\alpha}(\varepsilon), r_{\alpha}^{*}(\varepsilon): \alpha \leq \gamma\right\rangle$ is a legal partial play of $\partial_{0}^{\lambda}\left(\mathbb{Q}_{\varepsilon}\right)$ in which Complete uses her regular winning strategy $\mathbf{s t}_{\varepsilon}^{0} "$.

So, at stage $\gamma+1$ of the construction we apply Observation 2.3 to the sequence $\left\langle r_{\gamma}^{-} \sim_{\beta}\left\lceil\left[i_{\gamma}, i_{\gamma+1}\right): \beta<\delta\right\rangle \subseteq N \cap \mathbb{P}_{i_{\gamma+1}}\right.$ and the $\left(N, \mathbb{P}_{i_{\gamma+1}}\right)$-generic condition $r_{\gamma}^{*} \frown r \uparrow\left[i_{\gamma}, i_{\gamma+1}\right)$. This will give us conditions $r_{\gamma+1}^{-} \in N \cap \mathbb{P}_{i_{\gamma+1}}$ and $r_{\gamma+1} \in \mathbb{P}_{i_{\gamma+1}}$ so that the demands (i)-(iii) are satisfied. Then $r_{\gamma+1}^{*} \in \mathbb{P}_{i_{\gamma+1}}$ is chosen according to demand (iv), remembering that $\mathbf{s t}_{\varepsilon}^{0}$ are $\mathbb{P}_{\varepsilon}$-names for regular winning strategies. Next, at a limit stage $\gamma$ is limit then we first pick $r^{\prime} \in \mathbb{P}_{i_{\gamma}}$ that is stronger than all $r_{\alpha}^{*}$ for $\alpha<\gamma$ (exists by (iv)). This $r^{\prime}$ is $\left(N, \mathbb{P}_{i_{\gamma}}\right)$-generic, stronger than all $r_{\alpha}^{-}$for $\alpha<\gamma$ and stronger than all $q_{\beta} \backslash i_{\gamma}$ for $\beta<\delta$. So we may use Observation 2.3 to find $r_{\gamma}^{-}$and $r_{\gamma} \geq r^{\prime}$ as needed for (i)-(iii) and then pick $r_{\gamma}^{*}$ to fullfil (iv).

Let $r^{+} \in \mathbb{P}_{\zeta}$ be an upper bound of $\left\langle r_{\gamma}: \gamma<\operatorname{cf}(\zeta)\right\rangle$ (remember clause (iv) above); then also $r \leq r^{+}$. Now we are going to define a condition $q \in \mathbb{P}_{\zeta} \cap N$. We let

$$
\operatorname{dom}(q)=\bigcup\left\{\operatorname{dom}\left(r_{\gamma+1}^{-}\right) \cap\left[i_{\gamma}, i_{\gamma+1}\right): \gamma<\operatorname{cf}(\zeta)\right\},
$$

and for $\varepsilon \in \operatorname{dom}(q), i_{\gamma} \leq \varepsilon<i_{\gamma+1}$, we let $q(\varepsilon)$ be the $<_{\chi}^{*}$-first $\mathbb{P}_{\varepsilon}-$ name for the following object in $\mathbf{V}\left[G_{\mathbb{P}_{\varepsilon}}\right]$.

(A) If $r_{\gamma+1}^{-}(\varepsilon)\left[G_{\mathbb{P}_{\varepsilon}}\right]$ is an upper bound to $\left\{q_{\beta}(\varepsilon)\left[G_{\mathbb{P}_{\varepsilon}}\right]: \beta<\delta\right\}$ in $\underset{\sim}{\mathbb{Q}_{\varepsilon}}\left[G_{\mathbb{P}_{\varepsilon}}\right]$, then $q(\varepsilon)\left[G_{\mathbb{P}_{\varepsilon}}\right]=r_{\gamma+1}^{-}(\varepsilon)\left[G_{\mathbb{P}_{\varepsilon}}\right]$.

(B) If not (A) but $\left\{q_{\beta}(\varepsilon)\left[G_{\mathbb{P}_{\varepsilon}}\right]: \beta<\delta\right\}$ has an upper bound in $\underset{\sim}{\mathbb{Q}_{\varepsilon}}\left[G_{\mathbb{P}_{\varepsilon}}\right]$, then $q(\varepsilon)\left[G_{\mathbb{P}_{\varepsilon}}\right]$ is such a bound.

(C) If neither (A) nor (B), then $q(\varepsilon)\left[G_{\mathbb{P}_{\varepsilon}}\right]=q_{0}(\varepsilon)\left[G_{\mathbb{P}_{\varepsilon}}\right]$. 
It should be clear that $q \in \mathbb{P}_{\zeta} \cap N$. Now,

- $q \leq r^{+}$.

Why? By induction on $\varepsilon \in \zeta \cap N$ we show that $q \uparrow \varepsilon \leq r^{+} \uparrow \varepsilon$. Steps " $\varepsilon=0$ " and " $\varepsilon$ is limit" are clear, so suppose that we have proved $q \uparrow \varepsilon \leq r^{+} \uparrow \varepsilon, i_{\gamma} \leq \varepsilon<i_{\gamma+1}$ (and we are interested in restrictions to $\varepsilon+1)$. Assume that $G_{\mathbb{P}_{\varepsilon}} \subseteq \mathbb{P}_{\varepsilon}$ is a generic filter over $\mathbf{V}$ such that $r^{+}\left\lceil\varepsilon \in G_{\mathbb{P}_{\varepsilon}}\right.$. Since $q_{\beta}\left\lceil i_{\gamma+1} \leq r_{\gamma+1}^{-} \leq r_{\gamma+1} \leq r^{+}\right.$, we also have $q_{\beta}\left\lceil\varepsilon \in G_{\mathbb{P}_{\varepsilon}}\left(\right.\right.$ for $\beta<\delta$ ) and $r_{\gamma+1}^{-}\left\lceil\varepsilon \in G_{\mathbb{P}_{\varepsilon}}\right.$. Hence $r_{\gamma+1}^{-}(\varepsilon)\left[G_{\mathbb{P}_{\varepsilon}}\right]$ is an upper bound of $\left\{q_{\beta}(\varepsilon)\left[G_{\mathbb{P}_{\varepsilon}}\right]: \beta<\delta\right\}$. Therefore

$$
q(\varepsilon)\left[G_{\mathbb{P}_{\varepsilon}}\right]=r_{\gamma+1}^{-}(\varepsilon)\left[G_{\mathbb{P}_{\varepsilon}}\right] \leq r_{\gamma+1}(\varepsilon)\left[G_{\mathbb{P}_{\varepsilon}}\right] \leq r^{+}(\varepsilon)\left[G_{\mathbb{P}_{\varepsilon}}\right]
$$

(see (A) above) and we are done.

The proof of the Lemma will be finished once we show that

- $(\forall \beta<\delta)\left(q_{\beta} \leq q\right)$.

Why does this hold? By induction on $\varepsilon \in \zeta \cap N$ we show that $q_{\beta}\lceil\varepsilon \leq q\lceil\varepsilon$ for all $\beta<\delta$. Steps " $\varepsilon=0$ " and " $\varepsilon$ is limit" are as usual clear, so suppose that we have proved $q_{\beta}\left\lceil\varepsilon \leq q\left\lceil\varepsilon\right.\right.$ for $\beta<\delta, i_{\gamma} \leq \varepsilon<i_{\gamma+1}$ (and we are interested in the restrictions to $\varepsilon+1)$. Assume that $G_{\mathbb{P}_{\varepsilon}} \subseteq \mathbb{P}_{\varepsilon}$ is a generic filter over $\mathbf{V}$ such that $q\left\lceil\varepsilon \in G_{\mathbb{P}_{\varepsilon}}\right.$. Then, by the inductive hypothesis and the assumption (b) of the Lemma, we know that the sequence $\left\langle q_{\beta}(\varepsilon)\left[G_{\mathbb{P}_{\varepsilon}}\right]: \beta<\delta\right\rangle$ has an upper bound in $\mathbb{Q}_{\varepsilon}\left[G_{\mathbb{P}_{\varepsilon}}\right]$. Therefore, by $(\mathrm{A})+(\mathrm{B}), q(\varepsilon)\left[G_{\mathbb{P}_{\varepsilon}}\right] \geq q_{\beta}(\varepsilon)\left[G_{\mathbb{P}_{\varepsilon}}\right]$ for all $\beta<\delta$, and we are done.

Lemma 2.5. Suppose that $\zeta \in\left(\zeta^{*}+1\right) \cap N$ and conditions $r \in \mathbb{P}_{\zeta}$ and $p_{\alpha}, q_{\alpha} \in$ $\mathbb{P}_{\zeta} \cap N($ for $\alpha<\delta, \delta<\lambda)$ satisfy:

(i) $p_{\alpha} \leq q_{\alpha} \leq p_{\beta} \leq q_{\beta} \leq r$ for all $\alpha<\beta<\delta$,

(ii) for each $\xi \in \zeta \cap N$ and $\beta<\delta$ we have

$$
\begin{aligned}
q_{\beta}\left\lceil\xi \Vdash_{\mathbb{P}_{\xi}} \quad \text { " }\left\langle p_{\alpha}(\xi), q_{\alpha}(\xi): \alpha \leq \beta\right\rangle \text { is a legal partial play of } \partial_{0}^{\lambda}\left(\mathbb{Q}_{\xi}\right)\right. \\
\quad \text { in which Complete uses her regular winning strategy } \mathbf{s}_{\sim}^{0} \text { ", }
\end{aligned}
$$

(iii) either

$(\alpha) r$ is $\left(N, \mathbb{P}_{\zeta}\right)$-generic,

or

$(\beta) \zeta$ is a limit ordinal of cofinality $\operatorname{cf}(\zeta)<\lambda$ and for each $\xi \in \zeta \cap N$ the condition $r\left\lceil\xi\right.$ is $\left(N, \mathbb{P}_{\xi}\right)$-generic.

Let $\mathcal{I} \in N$ be an open dense subset of $\mathbb{P}_{\zeta}$ and $p^{-} \in N \cap \mathbb{P}_{\zeta}, p^{-} \leq r$. Then there are conditions $p^{\prime}, q^{\prime} \in \mathbb{P}_{\zeta} \cap N$ and $r^{\prime} \in \mathbb{P}_{\zeta}$ such that

(a) $r \leq r^{\prime}$ and $q_{\alpha} \leq p^{\prime} \leq q^{\prime} \leq r^{\prime}$ for all $\alpha<\delta$, and

(b) for each $\xi \in \zeta \cap N$ we have

$q^{\prime}\left\lceil\xi \Vdash_{\mathbb{P}_{\xi}} \quad\right.$ “ $\left\langle p_{\alpha}(\xi), q_{\alpha}(\xi): \alpha<\delta\right\rangle \frown\left\langle p^{\prime}(\xi), q^{\prime}(\xi)\right\rangle$ is a partial play of $\partial_{0}^{\lambda}\left(\mathbb{Q}_{\sim}\right)$ in which Complete uses her regular winning strategy $\mathbf{s t}_{\xi}^{0} "$,

(c) if we are under the assumption of (iii) $(\alpha)$, then also $p^{\prime} \in \mathcal{I}$,

(d) if we are under the assumption of (iii) $(\beta)$ and $q_{\alpha} \leq p^{-}$for all $\alpha<\delta$, then we still may require that $p^{-} \leq p^{\prime}$.

Proof. If we are under the assumption of (iii) $(\alpha)$, then the conclusion of the Lemma should be clear (including clause (c)).

So suppose that we are in the case described in $($ iii $)(\beta)$ and let $\left\langle i_{\gamma}: \gamma\langle\operatorname{cf}(\zeta)\rangle \subseteq\right.$ $N \cap \zeta$ be an increasing continuous sequence cofinal in $\zeta, i_{0}=0$. First, let us assume 
that the condition $p^{-} \in N \cap \mathbb{P}_{\zeta}$ satisfies $q_{\alpha} \leq p^{-} \leq r$ for all $\alpha<\delta$. By induction on $\gamma<\operatorname{cf}(\zeta)$ we will pick conditions $p^{\gamma}, q^{\gamma}, r^{\gamma}, s^{\gamma}$ so that:

$(*)_{1} p^{\gamma}, q^{\gamma} \in N \cap \mathbb{P}_{i_{\gamma}}, r^{\gamma}, s^{\gamma} \in \mathbb{P}_{i_{\gamma}}$,

$(*)_{2} p^{-}\left\lceil i_{\gamma} \leq p^{\gamma} \leq q^{\gamma}, r\left\lceil i_{\gamma} \leq r^{\gamma} \leq s^{\gamma}\right.\right.$ and $q^{\gamma} \leq r^{\gamma}$,

$(*)_{3} \quad q^{\gamma^{\prime}} \leq p^{\gamma}\left\lceil i_{\gamma^{\prime}}\right.$ and $s^{\gamma^{\prime}} \leq r_{\gamma}\left\lceil i_{\gamma^{\prime}}\right.$ for $\gamma^{\prime}<\gamma$,

$(*)_{4}$ if $i_{\gamma} \leq \xi<i_{\gamma+1}$ and $\gamma<\gamma^{*}<\operatorname{cf}(\zeta)$, then

$q^{\gamma^{*}} \mid \xi \Vdash_{\mathbb{P}_{\xi}}$ " the sequence $\left\langle p_{\alpha}(\xi), q_{\alpha}(\xi): \alpha<\delta\right\rangle \frown\left\langle p^{\gamma^{\prime}}(\xi), q^{\gamma^{\prime}}(\xi): \gamma<\gamma^{\prime} \leq \gamma^{*}\right\rangle$

is a legal partial play of $\partial_{0}^{\lambda}\left(\mathbb{Q}_{\xi}\right)$ in which

Complete uses her regular winning strategy $\mathbf{s t}_{\xi}^{0} "$,

and

$s^{\gamma^{*}} \mid \xi \Vdash_{\mathbb{P}_{\xi}} \quad$ " $\left\langle r^{\gamma^{\prime}}(\xi), s^{\gamma^{\prime}}(\xi): \gamma^{\prime} \leq \gamma^{*}\right\rangle$ is a legal partial play of $\partial_{0}^{\lambda}\left(\mathbb{Q}_{\xi}\right)$ in which Complete uses her regular winning strategy $\mathbf{s}_{\xi}^{0} "$.

When we arrive to a stage $\gamma<\operatorname{cf}(\zeta)$ of the construction, after having determined $p^{\gamma^{\prime}}, q^{\gamma^{\prime}}, r^{\gamma^{\prime}}, s^{\gamma^{\prime}}$ for $\gamma^{\prime}<\gamma$, we first pick a condition $r^{*} \in \mathbb{P}_{i_{\gamma}}$ stronger than all $s^{\gamma^{\prime}}$ for $\gamma^{\prime}<\gamma$ and such that $r \nmid i_{\gamma} \leq r^{*}$. [There is such $r^{*}$ by the second part of the demand $(*)_{4}$ at previous stages.] Then the condition $r^{*}$ is $\left(N, \mathbb{P}_{i_{\gamma}}\right)$-generic so we may use arguments as for the first part of the Lemma (case (iii) $(\alpha)$ ) to pick $p^{\gamma}, q^{\gamma}$ and $s^{\gamma} \geq r^{\gamma} \geq r^{*}$ so that demands in $(*)_{1}-(*)_{4}$ are satisfied.

After the construction is carried out we define $p^{\prime} \in \mathbb{P}_{\zeta}$ so that $\operatorname{dom}\left(p^{\prime}\right)=$ $\bigcup \operatorname{dom}\left(q^{\gamma}\right)$ and if $\xi \in \operatorname{dom}\left(p^{\prime}\right), i_{\gamma} \leq \xi<i_{\gamma+1}, \gamma<\operatorname{cf}(\zeta)$, then $p^{\prime}(\xi)$ is the $\gamma<\operatorname{cf}(\zeta)$

$<_{\chi}^{*}$-first $\mathbb{P}_{\xi}$-name such that the condition $p^{\prime}\left\lceil\xi\right.$ forces (in $\mathbb{P}_{\xi}$ ) that

$$
\text { " if } p^{-}(\xi) \leq p^{\gamma+1}(\xi) \text { then } p^{\prime}(\xi)=p^{\gamma+1}(\xi) \text {, otherwise } p^{\prime}(\xi)=p^{-}(\xi) \text { ". }
$$

Then $p^{\prime} \in N$ is a condition stronger than $p^{-}$(and so also stronger than all $q_{\alpha}$ for $\alpha<\delta)$. Let $q^{\prime} \in \mathbb{P}_{\zeta} \cap N$ be such that $\operatorname{dom}\left(q^{\prime}\right)=\operatorname{dom}\left(p^{\prime}\right)$ and for each $\xi \in \operatorname{dom}\left(q^{\prime}\right)$ we have

$$
q^{\prime} \mid \xi \Vdash_{\mathbb{P}_{\xi}} \quad \text { “ }\left\langle p_{\alpha}(\xi), q_{\alpha}(\xi): \alpha<\delta\right\rangle \frown\left\langle p^{\prime}(\xi), q^{\prime}(\xi)\right\rangle \text { is a partial play of } \partial_{0}^{\lambda}\left(\mathbb{Q}_{\xi}\right)
$$
in which Complete uses her regular winning strategy $\mathbf{s t}_{\xi}^{0} "$.

Plainly, $p^{\prime} \leq q^{\prime}$. Let $r^{\prime} \in \mathbb{P}_{\zeta}$ be an upper bound to $\left\langle s^{\gamma}: \gamma<\operatorname{cf}(\zeta)\right\rangle$ (exists by the second part of the demands in $\left.(*)_{4}\right)$. Then $r^{\prime} \geq r$. Now we show inductively that $r^{\prime}\left|\xi \geq q^{\prime}\right| \xi$ for $\xi<\zeta$. So suppose $\xi \in \operatorname{dom}\left(q^{\prime}\right), i_{\gamma} \leq \xi<i_{\gamma+1}$, and $q^{\prime}\left|\xi \leq r^{\prime}\right| \xi$. Then $r^{\prime} \mid \xi \Vdash p^{\prime}(\xi)=p^{\gamma+1}(\xi)$ (by $\left.(*)_{2}\right)$ and so also $r^{\prime} \mid \xi \Vdash q^{\prime}(\xi)=q^{\gamma+1}(\xi) \leq s^{\gamma+1}(\xi) \leq$ $r^{\prime}(\xi)$. Hence $p^{-} \leq p^{\prime} \leq q^{\prime} \leq r^{\prime}$.

If the condition $p^{-}$does not satisfy the assumptions of clause (d), then we may use Lemma 2.4 to find $q \in N \cap \mathbb{P}_{\zeta}$ and $r^{+} \in \mathbb{P}_{\zeta}$ such that $r \leq r^{+}, q \leq r^{+}$and $q_{\alpha} \leq q$ for all $\alpha<\delta$. Now carry out the above arguments for $q, r^{+}$in place of $p^{-}, r$.

Definition 2.6. (1) An $R S$-condition ${ }^{2}$ in $\mathbb{P}_{\zeta^{*}}$ is a pair $(p, w)$ such that $w \in$ $\left[\left(\zeta^{*}+1\right)\right]^{<\lambda}$ is a closed set, $0, \zeta^{*} \in w, p$ is a function with domain $\operatorname{dom}(p) \subseteq$ $\zeta^{*}$, and

$(\otimes)$ for every two successive members $\varepsilon^{\prime}<\varepsilon^{\prime \prime}$ of the set $w, p\left\lceil\left[\varepsilon^{\prime}, \varepsilon^{\prime \prime}\right)\right.$ is a $\mathbb{P}_{\varepsilon^{\prime}}$-name of an element of $\mathbb{P}_{\varepsilon^{\prime \prime}}$ whose support is included in the interval $\left[\varepsilon^{\prime}, \varepsilon^{\prime \prime}\right)$.

The family of all RS-conditions in $\mathbb{P}_{\zeta^{*}}$ is denoted by $\mathbb{P}_{\zeta^{*}}^{\mathrm{RS}}$.

\footnotetext{
2 the RS stands for "revised support"
} 
(2) If $(p, w) \in \mathbb{P}_{\zeta^{*}}^{\mathrm{RS}}$ and $G \subseteq \mathbb{P}_{\zeta^{*}}$ is a generic filter over $\mathbf{V}$, then we define $(p, w)^{G}=\bigcup\left\{\left(p\left\lceil\left[\varepsilon^{\prime}, \varepsilon^{\prime \prime}\right)\right)\left[G \cap \mathbb{P}_{\varepsilon^{\prime}}\right]: \varepsilon^{\prime}<\varepsilon^{\prime \prime}\right.\right.$ are successive members of $\left.w\right\}$

Note that $(p, w)^{G} \in \mathbb{P}_{\zeta^{*}}$. Also, we write $(p, w) \in^{\prime} G$ whenever $(p, w)^{G} \in G$. (3) If $\left(p_{1}, w_{1}\right),\left(p_{2}, w_{2}\right) \in \mathbb{P}_{\zeta^{*}}^{\mathrm{RS}}$, then we write $\left(p_{1}, w_{1}\right) \leq^{\prime}\left(p_{2}, w_{2}\right)$ whenever

$(\oplus)$ for every generic $G \subseteq \mathbb{P}_{\zeta^{*}}$ over $\mathbf{V}$,

$$
\text { if }\left(p_{2}, w_{2}\right) \in^{\prime} G \text { then }\left(p_{1}, w_{1}\right)^{G} \leq_{\mathbb{P}_{\zeta^{*}}}\left(p_{2}, w_{2}\right)^{G} \text {. }
$$

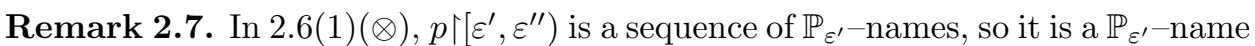
for some sequence. This (resulting) sequence is required to belong to $\mathbb{P}_{\varepsilon^{\prime \prime}}$, and just also to $\mathbf{V}$. We may therefore think about an RS-condition $(p, w)$ as follows. First, for every $\varepsilon \in w \cap \zeta^{*}$ we have

- a maximal antichain $\left\{p_{i}^{\varepsilon}: i \in I_{\varepsilon}\right\}$ of $\mathbb{P}_{\varepsilon}$, and

- a function $f^{\varepsilon}: I_{\varepsilon} \longrightarrow \mathbb{P}_{\zeta^{*}}$ such that $f^{\varepsilon}(i)$ is a condition with support included in the interval $[\varepsilon, \min (w \backslash(\varepsilon+1)))$.

(They describe a $\mathbb{P}_{\varepsilon}$-name for a condition in $\mathbb{P}_{\zeta^{*}}$ with support in the right interval.) Next, if $\varepsilon \leq \xi<\varepsilon^{\prime}$ where $\varepsilon, \varepsilon^{\prime}$ are successive members of $w$, then we may fix a $\mathbb{P}_{\xi^{-}}$ name $p(\xi)$ for a member of $\mathbb{Q}_{\xi}$ such that $p_{i}^{\varepsilon} \Vdash_{\mathbb{P}_{\xi}} p(\xi)=f^{\varepsilon}(i)(\xi)$. Note however that if we apply this approach to each $\xi$, we may not end up with a condition in $\mathbb{P}_{\zeta^{*}}$ because of the support! Nevertheless we will think of $p$ as a function on $\zeta^{*}$ where each $p(\xi)$ is a $\mathbb{P}_{\xi}$-name for a member of $\mathbb{Q}_{\sim}$.

Lemma 2.8. (1) If $(p, w) \in \mathbb{P}_{\zeta^{*}}^{\mathrm{RS}}$ and $q \in \mathbb{P}_{\zeta^{*}}$, then there is $q^{*} \in \mathbb{P}_{\zeta^{*}}$ stronger than $q$ and such that for each successive members $\varepsilon^{\prime}<\varepsilon^{\prime \prime}$ of $w$ the condition $q^{*}\left\lceil\varepsilon^{\prime}\right.$ decides $p\left\lceil\left[\varepsilon^{\prime}, \varepsilon^{\prime \prime}\right)\right.$ (i.e., $q^{*} \mid \varepsilon^{\prime} \Vdash " p\left\lceil\left[\varepsilon^{\prime}, \varepsilon^{\prime \prime}\right)=p_{\varepsilon^{\prime}, \varepsilon^{\prime \prime}}\right.$ " for some $p_{\varepsilon^{\prime}, \varepsilon^{\prime \prime}} \in$ $\left.\mathbb{P}_{\zeta^{*}}\right)$.

(2) For each $(p, w) \in \mathbb{P}_{\zeta^{*}}^{\mathrm{RS}}$ there is $q \in \mathbb{P}_{\zeta^{*}}$ such that $(p, w) \leq^{\prime}\left(q,\left\{0, \zeta^{*}\right\}\right)$.

(3) Let $\left(p_{i}, w_{i}\right) \in \mathbb{P}_{\zeta^{*}}^{\mathrm{RS}} \cap N$ (for $i<\delta<\lambda$ ), and $q \in \mathbb{P}_{\zeta^{*}} \cap N, r \in \mathbb{P}_{\zeta^{*}}$ be such that

$$
q \leq r \quad \text { and } \quad(\forall i<j<\delta)\left(\left(p_{i}, w_{i}\right) \leq^{\prime}\left(p_{j}, w_{j}\right) \leq^{\prime}\left(r,\left\{0, \zeta^{*}\right\}\right)\right) .
$$

Assume that either $r$ is $\left(N, \mathbb{P}_{\zeta^{*}}\right)$-generic, or $\zeta^{*}$ is a limit ordinal of cofinality $\operatorname{cf}\left(\zeta^{*}\right)<\lambda$ and for every $\zeta \in \zeta^{*} \cap N$ the condition $r\left\lceil\zeta\right.$ is $\left(N, \mathbb{P}_{\zeta}\right)$-generic. Then there are conditions $q^{\prime} \in N \cap \mathbb{P}_{\zeta^{*}}$ and $r^{\prime} \in \mathbb{P}_{\zeta^{*}}$ such that $q \leq q^{\prime} \leq r^{\prime}$, $r \leq r^{\prime}$ and $(\forall i<\delta)\left(\left(p_{i}, w_{i}\right) \leq^{\prime}\left(q^{\prime},\left\{0, \zeta^{*}\right\}\right)\right)$.

Proof. It is a slight generalization of [14, Proposition 3.6].

(1), (2) Straightforward (as $\mathbb{P}_{\zeta^{*}}$ is strategically $(<\lambda)$-complete).

(3) If $r$ is $\left(N, \mathbb{P}_{\zeta^{*}}\right)$-generic, then the conclusion is immediate. So let us consider the case when $\aleph_{0} \leq \operatorname{cf}\left(\zeta^{*}\right)<\lambda$ and $r\left\lceil\zeta\right.$ is $\left(N, \mathbb{P}_{\zeta}\right)$-generic for each $\zeta \in \zeta^{*} \cap N$.

Let $\left\langle i_{\gamma}: \gamma\left\langle\operatorname{cf}\left(\zeta^{*}\right)\right\rangle \subseteq N \cap \zeta^{*}\right.$ be a strictly increasing continuous sequence cofinal in $\zeta^{*}, i_{0}=0$. For $\gamma<\operatorname{cf}\left(\zeta^{*}\right)$ and $i<\delta$ we put $p_{i}^{\gamma}=p_{i}\left\lceil i_{\gamma}\right.$ and $w_{i}^{\gamma}=\left(w_{i} \cap i_{\gamma}\right) \cup\left\{i_{\gamma}\right\}$ (clearly $\left.\left(p_{i}^{\gamma}, w_{i}^{\gamma}\right) \in \mathbb{P}_{i_{\gamma}}^{\mathrm{RS}}\right)$.

Now, we inductively pick conditions $q_{\gamma}, q_{\gamma}^{+}, r_{\gamma}, r_{\gamma}^{+}$(for $\gamma<\operatorname{cf}\left(\zeta^{*}\right)$ ) so that

(i) $q_{\gamma}, q_{\gamma}^{+} \in \mathbb{P}_{i_{\gamma}} \cap N, r_{\gamma}, r_{\gamma}^{+} \in \mathbb{P}_{i_{\gamma}}$,

(ii) $q\left\lceil i_{\gamma} \leq q_{\gamma} \leq q_{\gamma}^{+} \leq r_{\gamma}, r\left\lceil i_{\gamma} \leq r_{\gamma} \leq r_{\gamma}^{+}\right.\right.$, and for each $\beta<\gamma$ we have

$$
q_{\beta}^{+} \leq q_{\gamma} \text { and } r_{\beta}^{+} \leq r_{\gamma}
$$


(iii) for every $\xi<i_{\gamma}$ we have

$$
\begin{aligned}
& q_{\gamma}^{+}\left\lceil\xi \Vdash_{\mathbb{P}_{\xi}} \quad \text { " }\left\langle q_{\beta}(\xi), q_{\beta}^{+}(\xi): \beta \leq \gamma\right\rangle \text { is a legal partial play of } \partial_{0}^{\lambda}\left(\mathbb{Q}_{\tilde{q}}\right)\right. \\
& \text { in which Complete uses her regular winning strategy } \mathbf{s t}_{\xi}^{0} " \text {, }
\end{aligned}
$$

(iv) if $i<\delta$ and $\varepsilon^{\prime}<\varepsilon^{\prime \prime}$ are two successive members of $w_{i}$ with $\varepsilon^{\prime}<i_{\gamma}$, then the condition $q_{\gamma}\left\lceil\varepsilon^{\prime}\right.$ decides $p_{i}\left\lceil\left[\varepsilon^{\prime}, \varepsilon^{\prime \prime}\right)\right.$ and $\left(p_{i}^{\gamma}, w_{i}^{\gamma}\right) \leq^{\prime}\left(q_{\gamma},\left\{0, i_{\gamma}\right\}\right)$.

At a limit stage $\gamma$ of the construction, we first pick an upper bound $r^{*} \in \mathbb{P}_{i_{\gamma}}$ to $\left\langle r_{\beta}^{+}: \beta<\gamma\right\rangle$ (exists by clause (iii) at previous stages). By (ii) we know that $r\left\lceil i_{\gamma} \leq r^{*}\right.$ so $r^{*}$ is $\left(N, \mathbb{P}_{i_{\gamma}}\right)$-generic. Consequently, we may use Lemma 2.5 to choose $q_{\gamma}, q_{\gamma}^{+} \in \mathbb{P}_{i_{\gamma}} \cap N$ and $r_{\gamma} \in \mathbb{P}_{i_{\gamma}}$ satisfying the relevant demands in (ii)+(iii), and then we pick $r_{\gamma}^{+} \geq r_{\gamma}$ so that the second part of (iii) holds. Note that the demand in (iv) will then follow by the inductive hypothesis.

Suppose now we are at a successor stage $\gamma+1$, so we assume $q_{\gamma}, q_{\gamma}^{+}, r_{\gamma}, r_{\gamma}^{+}$have been already chosen. We note that a condition $r^{*}=r_{\gamma}^{+} \frown\left(r \uparrow\left[i_{\gamma}, i_{\gamma+1}\right)\right) \in \mathbb{P}_{i_{\gamma+1}}$ is stronger than $r$ so it is $\left(N, \mathbb{P}_{i_{\gamma+1}}\right)$-generic. Therefore we may apply Lemma 2.5 to $r^{*}$, the sequence $\left\langle q_{\beta}, q_{\beta}^{+}: \beta \leq \gamma\right\rangle$ considered as a sequence of conditions in $\mathbb{P}_{i_{\gamma+1}}$ and a suitable open dense subset $\mathcal{I}$ of $\mathbb{P}_{i_{\gamma+1}}$ from $N$ to choose $q_{\gamma+1}, q_{\gamma+1}^{+}$and $r_{\gamma+1}, r_{\gamma+1}^{+}$ so that the demands in (i) - (iv) are satisfied. (For clause (iv) remember that by our assumptions we have $\left(p_{i}^{\gamma+1}, w_{i}^{\gamma+1}\right) \leq^{\prime}\left(r^{*},\left\{0, i_{\gamma+1}\right\}\right)$.)

Let $r^{*} \in \mathbb{P}_{\zeta^{*}}$ be stronger than all $r_{\gamma}$ 's. Apply Lemma 2.4 to the sequence $\left\langle q_{\gamma}^{+}: \gamma<\operatorname{cf}\left(\zeta^{*}\right)\right\rangle$ and the condition $r^{*}$ to choose $q^{\prime} \in N \cap \mathbb{P}_{\zeta^{*}}$ and $r^{\prime} \in \mathbb{P}_{\zeta^{*}}$ such that

$$
q^{\prime} \leq r^{\prime}, \quad r^{*} \leq r^{\prime}, \quad \text { and } \quad\left(\forall \gamma<\operatorname{cf}\left(\zeta^{*}\right)\right)\left(q_{\gamma}^{+} \leq q^{\prime}\right)
$$

Then $r^{\prime}, q^{\prime}$ are as required.

Proposition 2.9. Assume that

(1) $(p, w) \in \mathbb{P}_{\zeta^{*}}^{\mathrm{RS}}, \zeta \in w$ and $\sup (w \cap \zeta)<\xi<\zeta$,

(2) $w^{\prime} \subseteq \zeta^{*}+1$ is a closed set such that $\left|w^{\prime}\right|<\lambda$ and $w \cup\{\xi\} \subseteq w^{\prime}$,

(3) $\mathcal{\sim}$ is a $\mathbb{P}_{\zeta}$-name for an ordinal.

Then there are $p^{\prime}$ and $\mathcal{\tau}^{\prime}$ such that

(a) $\left(p^{\prime}, w^{\prime}\right) \in \mathbb{P}_{\zeta^{*}}^{\mathrm{RS}}$ and $(p, w) \leq^{\prime}\left(p^{\prime}, w^{\prime}\right)$,

(b) $p^{\prime} \uparrow \xi=p\left\lceil\xi\right.$ and $p^{\prime} \uparrow\left[\zeta, \zeta^{*}\right)=p \uparrow\left[\zeta, \zeta^{*}\right)$,

(c) $\underset{\sim}{\tau^{\prime}}$ is a $\mathbb{P}_{\xi}$-name, and

(d) if $G \subseteq \mathbb{P}_{\zeta^{*}}$ is generic over $\mathbf{V}$ and $\left(p^{\prime}, w^{\prime}\right) \in^{\prime} G$, then $\mathcal{\sim}^{\prime}[G]=\underset{\sim}{\tau}[G]$.

Proof. First we declare that $p^{\prime}\left\lceil\xi=p\left\lceil\xi\right.\right.$ and $p^{\prime} \uparrow\left[\zeta, \zeta^{*}\right)=p \uparrow\left[\zeta, \zeta^{*}\right)$. Now, $p \uparrow[\xi, \zeta)$ is a $\mathbb{P}_{\xi}$-name, so we may choose a maximal antichain $\mathcal{B} \subseteq \mathbb{P}_{\xi}$ and a function $g: \mathcal{B} \longrightarrow \mathbb{P}_{\zeta}$ such that for each $r \in \mathcal{B}$ we have

$(\alpha) \operatorname{dom}(g(r)) \subseteq[\xi, \zeta)$

( $\beta) r$ decides the value of $p \uparrow[\xi, \zeta)$ and $r \Vdash_{\mathbb{P}_{\xi}} p \uparrow[\xi, \zeta) \leq g(r)$,

$(\gamma) r \frown g(r)$ forces a value to $\underset{\sim}{\tau}$, say $r \frown g(r) \Vdash \underset{\sim}{\tau}=\tau(r)$.

Let ${\underset{\sim}{\tau}}^{\prime}$ be a $\mathbb{P}_{\xi}$-name such that $r \Vdash_{\mathbb{P}_{\xi}} \mathcal{\tau}^{\prime}=\tau(r)$ for each $r \in \mathcal{B}$, and let $p^{\prime} \uparrow[\xi, \zeta)$ be a $\mathbb{P}_{\xi}$-name such that $r \Vdash p^{\prime}\left\lceil[\xi, \zeta)=g(r)\right.$ for $r \in \mathcal{B}$. Then $\left(p^{\prime}, w^{\prime}\right)$ and $\mathcal{\sim}^{\prime}$ are as required in (a)-(d). 
It will be convenient for the proof of our main result 4.1 to look at RS-conditions also in a slightly different way.

Definition 2.10. Let $(p, w) \in \mathbb{P}_{\zeta^{*}}^{\mathrm{RS}}$. A standard representation of $(p, w)$ is a triple $(\mathcal{A}, f, w)$ such that

(i) $\mathcal{A} \subseteq \mathbb{P}_{\zeta^{*}}$ is a maximal antichain,

(ii) $f: \mathcal{A} \longrightarrow \mathbb{P}_{\zeta^{*}}$,

(iii) for each $r \in \mathcal{A}$, either $f(r) \leq r$ or for some $\varepsilon \in \operatorname{dom}(r)$ we have $f(r)|\varepsilon \leq r| \varepsilon$ and $r\left\lceil\varepsilon \Vdash_{\mathbb{P}_{\varepsilon}}\right.$ " $f(r)(\varepsilon), r(\varepsilon)$ are incompatible in $\mathbb{Q}_{\varepsilon}$ ",

(iv) if $r \in \mathcal{A}, \varepsilon \in w \cap \zeta^{*}$ and $\varepsilon^{\prime}=\min (w \backslash(\varepsilon+1))$, then $r\left\lceil\varepsilon \Vdash_{\mathbb{P}_{\varepsilon}} " p\left\lceil\varepsilon^{\prime}=f(r)\left\lceil\varepsilon^{\prime} "\right.\right.\right.$.

Observation 2.11. For each $(p, w) \in \mathbb{P}_{\zeta^{*}}^{\mathrm{RS}}$ and an open dense set $\mathcal{I} \subseteq \mathbb{P}_{\zeta^{*}}$ there is a standard representation $(\mathcal{A}, f, w)$ of $(p, w)$ such that $\mathcal{A} \subseteq \mathcal{I}$.

Observation 2.12. Assume that $(p, w) \in \mathbb{P}_{\zeta^{*}}^{\mathrm{RS}}$ and $(\mathcal{A}, f, w)$ is a standard representation of $(p, w)$.

(v) If $s, r \in \mathcal{A}, \varepsilon \in w \cap \zeta^{*}, \varepsilon^{\prime}=\min (w \backslash(\varepsilon+1))$ and the conditions $s\lceil\varepsilon$ and $r\left\lceil\varepsilon\right.$ are compatible in $\mathbb{P}_{\varepsilon}$, then $f(s)\left\lceil\varepsilon^{\prime}=f(r)\left\lceil\varepsilon^{\prime}\right.\right.$.

(vi) If $r \in \mathcal{A}, \varepsilon<\zeta^{*}$ and $f(r)\lceil\varepsilon \leq r\lceil\varepsilon$, then there is $s \in \mathcal{A}$ such that $r\lceil\varepsilon$ and $s\lceil\varepsilon$ are compatible and $f(s) \leq s$.

Proposition 2.13. Let $w \in\left[\zeta^{*}+1\right]^{<\lambda}$ be a closed set containing $0, \zeta^{*}$. Assume that $(\mathcal{A}, f, w)$ satisfies (i), (ii) and (iii) of Definition 2.10 and ( $v$ ) of Observation 2.12. Then there is $(p, w) \in \mathbb{P}_{\zeta^{*}}^{\mathrm{RS}}$ such that $(\mathcal{A}, f, w)$ is a standard representation of $(p, w)$.

Proof. Define $(p, w)$ so that

$$
r\left\lceil\varepsilon \Vdash _ { \mathbb { P } _ { \varepsilon } } p \left\lceil\left[\varepsilon, \varepsilon^{\prime}\right)=f(r) \uparrow\left[\varepsilon, \varepsilon^{\prime}\right)\right.\right.
$$

for $\varepsilon \in w \cap \zeta^{*}, \varepsilon^{\prime}=\min (w \backslash(\varepsilon+1))$ and $r \in \mathcal{A}$. Now check.

Definition 2.14. Let $\left(\mathcal{A}_{1}, f_{1}, w_{1}\right),\left(\mathcal{A}_{2}, f_{2}, w_{2}\right)$ be standard representations. We write $\left(\mathcal{A}_{1}, f_{1}, w_{1}\right) \preccurlyeq\left(\mathcal{A}_{2}, f_{2}, w_{2}\right)$ whenever

$(\circledast)_{1} \quad\left(\forall r \in \mathcal{A}_{2}\right)\left(\exists s \in \mathcal{A}_{1}\right)(s \leq r)$ and

$(\circledast)_{2}$ if $r \in \mathcal{A}_{2}, s \in \mathcal{A}_{1}, s \leq r$ and $f(r) \leq r$, then $f(s) \leq f(r)$.

Proposition 2.15. The relation $\preccurlyeq$ is transitive on standard representations.

Proof. Assume $\left(\mathcal{A}_{1}, f_{1}, w_{1}\right) \preccurlyeq\left(\mathcal{A}_{2}, f_{2}, w_{2}\right) \preccurlyeq\left(\mathcal{A}_{3}, f_{3}, w_{3}\right)$. Suppose that $r_{i} \in \mathcal{A}_{i}$ (for $i=1,2,3)$ are such that $r_{1} \leq r_{2} \leq r_{3}$ and $f_{3}\left(r_{3}\right) \leq r_{3}$. Then also $f_{2}\left(r_{2}\right) \leq f_{3}\left(r_{3}\right)$ and consequently $f_{2}\left(r_{2}\right)$ and $r_{2}$ are compatible. By 2.10(iii) we have $f_{2}\left(r_{2}\right) \leq r_{2}$ and therefore also $f_{1}\left(r_{1}\right) \leq f_{2}\left(r_{2}\right)$. Together $f_{1}\left(r_{1}\right) \leq f_{2}\left(r_{2}\right) \leq f_{3}\left(r_{3}\right)$, and the transitivity of $\preccurlyeq$ follows.

Observation 2.16. Suppose that $\left(p_{1}, w_{1}\right),\left(p_{2}, w_{2}\right) \in \mathbb{P}_{\zeta^{*}}^{\mathrm{RS}}$ and let $\left(\mathcal{A}_{1}, f_{1}, w_{1}\right)$ and $\left(\mathcal{A}_{2}, f_{2}, w_{2}\right)$ be their standard representations. Assume $\left(\mathcal{A}_{1}, f_{1}, w_{1}\right) \preccurlyeq\left(\mathcal{A}_{2}, f_{2}, w_{2}\right)$. Then $\left(p_{1}, w_{1}\right) \leq^{\prime}\left(p_{2}, w_{2}\right)$.

Proposition 2.17. Let $\left(p_{1}, w_{1}\right),\left(p_{2}, w_{2}\right) \in \mathbb{P}_{\zeta^{*}}^{\mathrm{RS}}$ be such that $\left(p_{1}, w_{1}\right) \leq^{\prime}\left(p_{2}, w_{2}\right)$. Suppose that $\left(\mathcal{A}_{1}, f_{1}, w_{1}\right)$ is a standard representation of $\left(p_{1}, w_{1}\right)$. Then there is a standard representation $\left(\mathcal{A}_{2}, f_{2}, w_{2}\right)$ for $\left(p_{2}, w_{2}\right)$ such that $\left(\mathcal{A}_{1}, f_{1}, w_{1}\right) \preccurlyeq\left(\mathcal{A}_{2}, f_{2}, w_{2}\right)$. 
Proof. Choose a standard representation $\left(\mathcal{A}_{2}, f_{2}, w_{2}\right)$ of $\left(p_{2}, w_{2}\right)$ such that $(\forall s \in$ $\left.\mathcal{A}_{2}\right)\left(\exists r \in \mathcal{A}_{1}\right)(r \leq s)$ (possible by Observation 2.11). Then plainly the demand $(\circledast)_{2}$ of Definition 2.14 is also satisfied, so $\left(\mathcal{A}_{1}, f_{1}, w_{1}\right) \preccurlyeq\left(\mathcal{A}_{2}, f_{2}, w_{2}\right)$.

2.2. The Forcings. Let us recall the definitions of the main forcing notions we are interested in. These forcing notions generalize the well known classical forcings with trees used in the set theory of the reals. Thus both $\mathbb{Q}_{\lambda}^{1}$ and $\mathbb{Q}_{\lambda}^{2}$ (defined below in 2.18) represent possible generalizations of Miller's rational perfect set forcing, see Miller [13]. The Laver forcing notion (see Laver [1]) is generalized by forcings $\mathbb{Q}_{\lambda}^{4}$. Due to flexibility of our parameters, the schemes presented here generalize the Cohen and the Sacks forcing notions as well, see Remark 2.19. The many "bounded" variations of the classical forcings with trees have suitable nice generalizations if $\lambda$ is inaccessible, see Definition 2.20. In Definition 2.22(1) we will introduce generalizations of the Silver forcing notion (or perhaps rather the Grigorieff forcing, see [6, §3]). Finally, relatives of the Hechler forcing (Hechler [7) and Eventually Different Real forcing (Miller [12, §5]) are introduced in Definition $2.22(2,3)$.

Definition 2.18. Suppose that $\bar{E}=\left\langle E_{t}: t \in{ }^{<\lambda} \lambda\right\rangle$ is a system of $(<\lambda)$-complete filters on a regular cardinal $\lambda$ and $E$ is a normal filter on $\lambda$. We define forcing notions $\mathbb{Q}^{\ell, \bar{E}}$ (for $\left.\ell=1,2,3,4\right)$ and $\mathbb{Q}_{E}^{1, \bar{E}}$ and $\mathbb{Q}_{E}^{3, \bar{E}}$ as follows.

(1) A condition in $\mathbb{Q}^{2, \bar{E}}$ is a complete $\lambda$-tree $T \subseteq{ }^{<\lambda} \lambda$ such that

(a) if $t \in T$, then either $\left|\operatorname{succ}_{T}(t)\right|=1$ or $\operatorname{succ}_{T}(t) \in E_{t}$, and

(b) $(\forall t \in T)(\exists s \in T)\left(t \triangleleft s \&\left|\operatorname{succ}_{T}(s)\right|>1\right)$, and

(c) $)^{2}$ if $j<\lambda$ and a sequence $\left\langle t_{i}: i<j\right\rangle \subseteq T$ is $\triangleleft$-increasing, $\left|\operatorname{succ}_{T}\left(t_{i}\right)\right|>1$ for all $i<j$ and $t=\bigcup_{i<j} t_{i}$, then $(t \in T$ and $)\left|\operatorname{succ}_{T}(t)\right|>1$,

the order $\leq$ of $\mathbb{Q}^{2, \bar{E}}$ is the inverse inclusion, i.e., $T_{1} \leq T_{2}$ if and only if $T_{1}, T_{2} \in \mathbb{Q}^{2, \overline{\bar{E}}}$ and $T_{2} \subseteq T_{1}$.

(2) Forcing notions $\mathbb{Q}^{1, \bar{E}}, \mathbb{Q}^{3, \bar{E}}, \mathbb{Q}^{4, \bar{E}}$ are defined analogously, but the demand $(\mathrm{c})^{2}$ is replaced by the respective $(\mathrm{c})^{\ell}$ :

$(\mathrm{c})^{1}$ for every $\lambda$-branch $\eta \in \lim _{\lambda}(T)$ the set

$$
\left\{\alpha<\lambda: \mid \operatorname{succ}_{T}(\eta\lceil\alpha) \mid>1\}\right.
$$

includes a closed unbounded set.

$(c)^{3}$ for some closed unbounded set $C \subseteq \lambda$ consisting of limit ordinals we have

$$
(\forall t \in T)\left(\operatorname{lh}(t) \in C \Leftrightarrow\left|\operatorname{succ}_{T}(t)\right|>1\right),
$$

$(\mathrm{c})^{4}(\forall t \in T)\left(\operatorname{root}(T) \triangleleft t \Rightarrow\left|\operatorname{succ}_{T}(t)\right|>1\right)$.

(3) The forcing notions $\mathbb{Q}_{E}^{1, \bar{E}}$ and $\mathbb{Q}_{E}^{3, \bar{E}}$ are defined like $\mathbb{Q}^{1, \bar{E}}$ and $\mathbb{Q}^{3, \bar{E}}$, but in demands (c) $)^{1}$ and $(\mathrm{c})^{3}$ we replace "a closed unbounded set" by "a set of limit ordinals belonging to the filter $E$ ".

(4) If each $E_{t}$ is the club filter of $\lambda$ (for all $t \in{ }^{<\lambda} \lambda$ ), then we omit $\bar{E}$ and we write $\mathbb{Q}_{\lambda}^{\ell}$ instead of $\mathbb{Q}^{\ell, \bar{E}}$.

Remark 2.19. (1) Note that our definition of $\mathbb{Q}_{\lambda}^{3}$ slightly differs from the one in [19, however the forcing defined here is a dense subset of the one defined there. 
(2) The forcing notion $\mathbb{Q}^{2, \bar{E}}$ was studied by Brown and Groszek 2$]$ who described when this forcing adds a generic of minimal degree.

(3) Remember that in Definition 2.18 we allow the filters $E_{t}$ to be principal. Thus if $E_{t}=\{\lambda\}$ for each $t \in{ }^{<\lambda} \lambda$, then $\mathbb{Q}^{4, \bar{E}}$ is the $\lambda$-Cohen forcing $\mathbb{C}_{\lambda}$ and $\mathbb{Q}^{2, \bar{E}}$ is the forcing $\mathbb{D}_{\lambda}$ from [14, Proposition 4.10]. If for each $t \in \epsilon^{<\lambda} \lambda$ we let $E_{t}$ be the filter of all subsets of $\lambda$ including $\{0,1\}$, then the forcing notion $\mathbb{Q}^{2, \bar{E}}$ will be equivalent with Kanamori's $\lambda$-Sacks forcing of [10, Definition $1.1]$.

(4) A relative of $\mathbb{Q}_{\lambda}^{2}$ was used in iterations in Friedman and Zdomskyy [5] and Friedman, Honzik and Zdomskyy [4. It was called Miller $(\lambda)$ there and the main difference between the two forcings is in condition [5, Definition $2.1(\mathrm{vi})]$.

(5) The property introduced in this paper does not "capture" $\mathbb{Q}_{\lambda}^{1}$. In a subsequent work we will modify it to include more forcings of the form $\mathbb{Q}_{E}^{1, \bar{E}}$. However one should note that the forcing notions $\mathbb{Q}_{\lambda}^{1}$ and $\mathbb{Q}_{\lambda}^{2}$ are very similar. If in the demand $2.18(2)(c)^{1}$ we replace "includes a closed unbounded set" with "is closed unbounded" then we clearly get an equivalent definition of $\mathbb{Q}_{\lambda}^{2}$. In [19, Section 4] we showed that consistently the forcing notions $\mathbb{Q}_{\lambda}^{1}$ and $\mathbb{Q}_{\lambda}^{2}$ are equivalent, but also consistently they are not equivalent.

Definition 2.20. Assume that

- $\lambda$ is weakly inaccessible, $\varphi: \lambda \longrightarrow \lambda$ is a strictly increasing function such that each $\varphi(\alpha)$ is a regular uncountable cardinal and $\alpha<\varphi(\alpha)$ (for $\alpha<\lambda$ ),

- $\bar{F}=\left\langle F_{t}: t \in \bigcup_{\alpha<\lambda} \prod_{\beta<\alpha} \varphi(\beta)\right\rangle$ where $F_{t}$ is a $<\varphi(\alpha)$-complete filter on $\varphi(\alpha)$ whenever $t \in \prod_{\beta<\alpha} \varphi(\beta), \alpha<\lambda$,

- $E$ is a normal filter on $\lambda$.

(1) We define a forcing notion $\mathbb{Q}_{\varphi, \bar{F}}^{2}$ as follows.

A condition in $\mathbb{Q}_{\varphi, \bar{F}}^{2}$ is a complete $\lambda$-tree $T \subseteq \bigcup_{\alpha<\lambda} \prod_{\beta<\alpha} \varphi(\beta)$ such that

(a) for every $t \in T$, either $\left|\operatorname{succ}_{T}(t)\right|=1$ or $\operatorname{succ}_{T}(t) \in F_{t}$, and

(b) $(\forall t \in T)(\exists s \in T)\left(t \triangleleft s \&\left|\operatorname{succ}_{T}(s)\right|>1\right)$, and

$(\mathrm{c})^{2}$ if $j<\lambda$ and a sequence $\left\langle t_{i}: i<j\right\rangle \subseteq T$ is $\triangleleft$-increasing, $\left|\operatorname{succ}_{T}\left(t_{i}\right)\right|>1$ for all $i<j$ and $t=\bigcup_{i<j} t_{i}$, then $(t \in T$ and $)\left|\operatorname{succ}_{T}(t)\right|>1$.

The order of $\mathbb{Q}_{\varphi, \bar{F}}^{2}$ is the reverse inclusion.

(2) Forcing notions $\mathbb{Q}_{\varphi, \bar{F}}^{\ell}$ for $\ell=1,3,4$ are defined similarly, but the demand $(\mathrm{c})^{2}$ is replaced by the respective $(\mathrm{c})^{\ell}$ :

$(\mathrm{c})^{1}$ for every $\eta \in \lim _{\lambda}(T)$ the set $\left\{\alpha<\lambda: \mid \operatorname{succ}_{T}(\eta\lceil\alpha) \mid>1\}\right.$ contains a closed unbounded subset of $\lambda$.

$(c)^{3}$ for some closed unbounded set $C \subseteq \lambda$ consisting of limit ordinals we have

$$
(\forall t \in T)\left(\operatorname{lh}(t) \in C \Leftrightarrow\left|\operatorname{succ}_{T}(t)\right|>1\right) .
$$

$(\mathrm{c})^{4}(\forall t \in T)\left(\operatorname{root}(T) \triangleleft t \Rightarrow\left|\operatorname{succ}_{T}(t)\right|>1\right)$.

(3) Replacing "a closed unbounded set" in $(c)^{1}$ and (c) $)^{3}$ by "a set of limit ordinals belonging to the filter $E$ " will define forcing notions $\mathbb{Q}_{\varphi, \bar{F}, E}^{1}$ and $\mathbb{Q}_{\varphi, \bar{F}, E}^{3}$, respectively. 
Remark 2.21. If $\lambda$ is strongly inaccessible and $\varphi, \bar{F}$ are as in 2.20 , then $\mathbb{Q}_{\varphi, \bar{F}}^{3}$ is a dense subset of $\mathbb{Q}_{\varphi, \bar{F}}^{2}$.

Definition 2.22. (1) Assume that $\psi: \lambda \longrightarrow(\lambda+1) \backslash\{0,1\}$ and $E$ is a normal filter on $\lambda$. We define a forcing notion $\mathbb{S}_{E}^{\psi}$ as follows.

A condition in $\mathbb{S}_{E}^{\psi}$ is a function $p$ such that $\operatorname{dom}(p) \subseteq \lambda, \lambda \backslash \operatorname{dom}(p) \in E$ and $p(i)<\psi(i)$ for each $i \in \operatorname{dom}(p)$,

the order $\leq$ of $\mathbb{S}_{E}^{\psi}$ is the inclusion, i.e., $p \leq q$ if and only if $p, q \in \mathbb{S}_{E}^{\psi}$ and $p \subseteq q$.

For $p \in \mathbb{S}_{E}^{\psi}$ we also set $\operatorname{rt}(p)=\min (\lambda \backslash \operatorname{dom}(p))$ (the notation rt points to analogy with "the length of the root").

(2) A condition in a forcing notion $\mathbb{H}_{\lambda}$ is a pair $(s, g)$ such that $s \in{ }^{\lambda>} \lambda$ and $g \in{ }^{\lambda} \lambda$.

The order $\leq$ of $\mathbb{H}_{\lambda}$ is defined by: $(s, g) \leq\left(s^{\prime}, g^{\prime}\right)$ if and only if $\left((s, g),\left(s^{\prime}, g^{\prime}\right) \in\right.$ $\mathbb{H}_{\lambda}$ and $) s \unlhd s^{\prime}, g(\alpha) \leq s^{\prime}(\alpha)$ for each $\alpha \in\left[\operatorname{lh}(s), \operatorname{lh}\left(s^{\prime}\right)\right)$ and $g(\alpha) \leq g^{\prime}(\alpha)$ for all $\alpha \in\left[\ln \left(s^{\prime}\right), \lambda\right)$.

(3) A condtion in a forcing notion $\mathbb{E}_{\lambda}$ is a pair $(s, \bar{G})$ such that $s \in{ }^{\lambda>} \lambda$, $\bar{G}=\left\langle G_{\beta}: \beta<\lambda\right\rangle$ and for some $\mu<\lambda$, for every $\beta<\lambda$ we have $G_{\beta} \in[\lambda] \leq \mu$. The order $\leq$ of $\mathbb{E}_{\lambda}$ is defined by: $(s, \bar{G}) \leq\left(s^{\prime}, \bar{G}^{\prime}\right)$ if and only if $\left((s, \bar{G}),\left(s^{\prime}, \bar{G}^{\prime}\right) \in\right.$ $\mathbb{E}_{\lambda}$ and $s \unlhd s^{\prime}, s^{\prime}(\alpha) \notin G_{\alpha}$ for each $\alpha \in\left[\operatorname{lh}(s), \ln \left(s^{\prime}\right)\right)$ and $G_{\alpha} \subseteq G_{\alpha}^{\prime}$ for all $\alpha \in\left[\ln \left(s^{\prime}\right), \lambda\right)$.

Observation 2.23. Assume $\lambda^{<\lambda}=\lambda$.

(1) For $\bar{E}$ as in 2.18 and $\ell \in\{1,2,3,4\}$, the forcing notion $\mathbb{Q}^{\ell, \bar{E}}$ is $(<\lambda)$-lubcomplete (i.e., increasing sequences of length $<\lambda$ have least upper bounds). Likewise for $\mathbb{Q}_{E}^{1, \bar{E}}$ and $\mathbb{Q}_{E}^{3, \bar{E}}$.

(2) For $\varphi, \bar{F}$ as in 2.20 and $\ell \in\{1,2,3,4\}$, the forcing notion $\mathbb{Q}_{\varphi, \bar{F}}^{\ell}$ is strategically $(<\lambda)$-complete. Moreover, if $\bar{T}=\left\langle T_{\alpha}: \alpha<\delta\right\rangle \subseteq \mathbb{Q}_{\varphi, \bar{F}}^{\ell}$ is $\leq_{\mathbb{Q}_{\varphi, \bar{F}}^{\ell}}-$ increasing and $\operatorname{root}\left(T_{\alpha}\right) \triangleleft \operatorname{root}\left(T_{\beta}\right)$ for $\alpha<\beta<\delta<\lambda$, then $\bigcap_{\alpha<\delta} T_{\alpha} \in \mathbb{Q}_{\varphi, \bar{F}}^{\ell}$ is the least upper bound to $\bar{T}$. Similarly for $\mathbb{Q}_{\varphi, \bar{F}, E}^{1}$ and $\mathbb{Q}_{\varphi, \bar{F}, E}^{3}$.

(3) For $E$ and $\psi$ as in 2.22, the forcing notion $\mathbb{S}_{E}^{\psi}$ is $(<\lambda)$-lub-complete.

(4) The forcing notions $\mathbb{H}_{\lambda}$ and $\mathbb{E}_{\lambda}$ are $(<\lambda)$-lub-complete.

Arguably, one of the most important properties of forcing notions with trees in the set theory of the reals is the possibility to have fusion for suitable $\omega$-sequences of trees. Similar properties hold for our forcing notions (with respect to $\lambda$-sequences of conditions). The next two lemmas are actually fusion lemmas exemplifying this similarity.

Lemma 2.24. Assume that

- either $\bar{E}$ and $E$ are as in $2.18,1 \leq \ell \leq 4$, and $\mathbb{P}=\mathbb{Q}^{\ell, \bar{E}}$, or $\mathbb{P}=\mathbb{Q}_{E}^{1, \bar{E}}$, or $\mathbb{P}=\mathbb{Q}_{E}^{3, \bar{E}}$

- or $\lambda, \varphi, \bar{F}$ and $E$ are as in $2.20,1 \leq \ell \leq 4$ and $\mathbb{P}=\mathbb{Q}_{\varphi, \bar{F}}^{\ell}$ or $\mathbb{P}=\mathbb{Q}_{\varphi, \bar{F}, E}^{1}$ or $\mathbb{P}=\mathbb{Q}_{\varphi, \bar{F}, E}^{3}$

Suppose that $\gamma \leq \lambda$ and $T^{\delta} \in \mathbb{P}$ for $\delta<\gamma$ are such that

(i) $T^{\delta+1} \subseteq T^{\delta}$ and $T^{\delta} \cap{ }^{\delta} \lambda=T^{\delta+1} \cap{ }^{\delta} \lambda$,

(ii) if $\delta$ is limit, then $T^{\delta}=\bigcap_{i<\delta} T^{i}$, 
(iii) if $t \in T^{\delta} \cap{ }^{\delta} \lambda$ and $\left|\operatorname{succ}_{T^{\delta}}(t)\right|>1$, then $\left|\operatorname{succ}_{T^{\delta+1}}(t)\right|>1$.

Then $T^{\gamma} \stackrel{\text { def }}{=} \bigcap_{\delta<\gamma} T^{\delta} \in \mathbb{P}$.

Proof. For $\mathbb{Q}_{\lambda}^{\ell}$ it was proved in [19, Lemma 3.5]; for other cases the arguments are essentially the same.

Lemma 2.25. Assume that $\psi, E$ are as in 2.22. Suppose that $p_{\delta} \in \mathbb{S}_{E}^{\psi}$ for $\delta<\lambda$ are such that

(i) $p_{\delta} \subseteq p_{\delta+1}$ and $p_{\delta} \uparrow(\delta+1)=p_{\delta+1} \uparrow(\delta+1)$, and

(ii) if $\delta$ is limit, then $p_{\delta}=\bigcup_{i<\delta} p_{i}$.

Then $p_{\lambda} \stackrel{\text { def }}{=} \bigcup_{\delta<\lambda} p_{\delta} \in \mathbb{S}_{E}^{\psi}$.

Proof. Clearly $p_{\lambda}$ is a function with $\operatorname{dom}(p)=\bigcup_{\delta<\lambda} \operatorname{dom}\left(p_{\delta}\right) \subseteq \lambda$ and it satisfies $p_{\lambda}(i)<\psi(i)$ for each $i \in \operatorname{dom}\left(p_{\lambda}\right)$. We should to argue that $\lambda \backslash \operatorname{dom}\left(p_{\lambda}\right) \in$ $E$. For this we just note that if $\delta \in \triangle \triangle_{\alpha<\lambda}\left(\lambda \backslash \operatorname{dom}\left(p_{\alpha}\right)\right)$ is a limit ordinal, then $\delta \notin \bigcup_{\alpha<\delta} \operatorname{dom}\left(p_{\alpha}\right)=\operatorname{dom}\left(p_{\delta}\right)$ (by (ii)) and hence $\delta \notin \operatorname{dom}\left(p_{\delta+1}\right)$ (by (i)). The assumption (i) implies also that $\delta \notin \operatorname{dom}\left(p_{\lambda}\right)$.

\section{Sequential PuRity With Diamonds}

For the rest of the paper we assume the following Context.

Context 3.1. (1) $\lambda$ is a regular uncountable cardinal, $\lambda^{<\lambda}=\lambda$.

(2) $D$ is a normal filter on $\lambda$.

(3) A set $\mathcal{S} \in D^{+}$contains all successor ordinals below $\lambda, 0 \notin \mathcal{S}$ and $\lambda \backslash \mathcal{S}$ is unbounded in $\lambda$. For an ordinal $\gamma<\lambda$ we define $\mathcal{S}[\gamma]=\mathcal{S} \backslash\{\delta \leq \gamma$ : $\delta$ is limit $\}$.

(4) $\mathcal{R}$ is the closure of $\lambda \backslash \mathcal{S}$ and $\bar{\gamma}=\left\langle\gamma_{\alpha}: \alpha<\lambda\right\rangle$ is the increasing enumeration of $\mathcal{R}$ (so the sequence $\bar{\gamma}$ is increasing continuous, $\gamma_{0}=0$ and all other terms of $\bar{\gamma}$ are limit ordinals).

Definition 3.2. A sequence $\bar{f}=\left\langle f_{\delta}: \delta \in \mathcal{S}\right\rangle$ is a $(D, \mathcal{S})$-diamond if $f_{\delta} \in{ }^{\delta} \delta$ for $\delta \in \mathcal{S}$ and $\left(\forall \eta \in{ }^{\lambda} \lambda\right)\left(\left\{\delta \in \mathcal{S}: f_{\delta} \triangleleft \eta\right\} \in D^{+}\right)$.

Observation 3.3. Let $\mathbb{P}$ be a strategically $(<\lambda)$-complete forcing notion, $D$ be a normal filter on $\lambda$.

(1) $\Vdash_{\mathbb{P}}$ " The family of all supersets of diagonal intersections of members of $D^{\mathbf{V}}$ is a (proper) normal filter on $\lambda$ ". (Abusing our notation, the (name for the) normal filter generated by $D$ in $\mathbf{V}^{\mathbb{P}}$ will also be denoted by $D$ or sometimes by $D^{\mathbf{V}\left[G_{\mathbb{P}}\right]}$.)

(2) If $\bar{f}$ is a $(D, \mathcal{S})$-diamond, then $\Vdash_{\mathbb{P}}$ " $\bar{f}$ is a $\left(D^{\mathbf{V}\left[G_{\mathbb{P}}\right]}, \mathcal{S}\right)$-diamond".

A pure extension of a tree is a subtree with the same root. This concept appears in several places in the classical forcing with trees, e.g., in the Laver forcing every sentence can be decided by passing to a pure extension of a condition. The relations $\leq_{\text {pr }}$ of pure extensions were important ingredients of the properties studied in [17, Section 2] and [19. The property introduced in the present paper also involves pure extensions, but for technical reasons they are considered for bounds of increasing 
sequences of conditions. However, for our "test tree forcing notions" the intuitions behind the sequential purity $R^{\mathrm{pr}}$ introduced in Definition 3.4 below should be that it is essentially the same as $\leq_{\text {pr }}$. For instance, in $\mathbb{Q}_{\lambda}^{2}$ we say that $T \in \mathbb{Q}_{\lambda}^{2}$ is a pure bound to $\bar{T}=\left\langle T_{\alpha}: \alpha<\delta\right\rangle$ if $\bar{T}$ is an increasing sequence of conditions from $\mathbb{Q}_{\lambda}^{2}$, $\delta<\lambda$ is a limit ordinal, $\alpha \leq \operatorname{lh}\left(\operatorname{root}\left(T_{\alpha}\right)\right)<\delta$ and $T$ is a pure extension of $\bigcap_{\alpha<\delta} T_{\alpha}$.

(We will write then $\bar{T} R^{\text {pr }} T$.) Plainly, an increasing sequence of conditions in $\mathbb{Q}_{\lambda}^{2}$ of length $<\lambda$, all of them with the same root $t$, has an upper bound with the root $t$. Therefore, the relation $R^{\text {pr }}$ introduced above is actually a $\lambda$-sequential ${ }^{+}$purity on $\mathbb{Q}_{\lambda}^{2}$.

Definition 3.4. Let $\mathbb{Q}$ be a forcing notion. A binary relation $R^{\text {pr }}$ is called $a$ $\lambda$-sequential purity on $\mathbb{Q}$ whenever $\bar{r} R^{\text {pr }} r$ implies

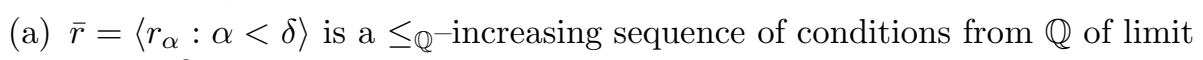
length $\delta<\lambda$, and

(b) $r \in \mathbb{Q}$ is an upper bound of $\bar{r}$ (i.e., $r_{\alpha} \leq_{\mathbb{Q}} r$ for all $\alpha<\delta$ ).

If, additionally, the relation $R^{\mathrm{pr}}$ satisfies

(c) if $\bar{r}=\left\langle r_{\alpha}: \alpha<\delta\right\rangle, \bar{r} R^{\text {pr }} s_{\beta}$ for $\beta<\xi, \xi<|\delta|^{+}$and $s_{\beta} \leq s_{\gamma}$ for $\beta<\gamma<\xi$, then there is a condition $s \in \mathbb{Q}$ stronger than all $s_{\beta}$ (for $\beta<\xi$ ) and such that $\bar{r} R^{\mathrm{pr}} s$,

then we say that $R^{\mathrm{pr}}$ is a $\lambda$-sequential ${ }^{+}$purity on $\mathbb{Q}$.

The next definition introduces the main technical concept of the paper: the game $\partial_{\gamma}^{\mathcal{S}}\left(r, N, h, \mathbb{Q}, R^{\mathrm{pr}}, \bar{f}, \bar{q}\right)$ between two players, Generic and Antigeneric. Suppose that $\mathbb{Q}=\mathbb{Q}_{\lambda}^{2}$. In a play of $\partial_{\gamma}^{\mathcal{S}}$ the two players construct two descending (with respect to the inclusion) sequences of trees $\left\langle T_{i}^{-}: i<\lambda\right\rangle \subseteq \mathbb{Q}_{\lambda}^{2} \cap N$ and $\left\langle T_{i}: i<\lambda\right\rangle \subseteq \mathbb{Q}_{\lambda}^{2}$ with the property that oftentimes $T_{i} \subseteq T_{i}^{-}$. If $i \in S[\gamma]$ then the trees $T_{i}^{-}, T_{i}$ are chosen by Generic, otherwise they are picked by Antigeneric. It is not unreasonable to think that for $i<j$ we have

$$
\operatorname{root}\left(T_{i}^{-}\right) \unlhd \operatorname{root}\left(T_{i}\right) \triangleleft \operatorname{root}\left(T_{j}^{-}\right) \unlhd \operatorname{root}\left(T_{j}\right) .
$$

Then for many limit $\delta<\lambda$, a diamond sequence $\bar{f}$ guesses (via some coding function $h$ ) the sequence $\left\langle T_{i}^{-}: i<\delta\right\rangle$ and $\bigcap_{i<\delta} T_{i}^{-} \in \mathbb{Q}_{\lambda}^{2} \cap N$ is a tree with the root

$$
\operatorname{root}\left(\bigcap_{i<\delta} T_{i}^{-}\right)=\bigcup_{i<\delta} \operatorname{root}\left(T_{i}^{-}\right)=\bigcup_{i<\delta} \operatorname{root}\left(T_{i}\right)
$$

The task of Generic will be to make sure that oftentimes for these limit $\delta$ it holds that

$$
T_{\delta} \subseteq q_{\delta} \text { and } \operatorname{root}\left(T_{\delta}\right)=\operatorname{root}\left(q_{\delta}\right)=\operatorname{root}\left(\bigcap_{i<\delta} T_{i}^{-}\right)
$$

Definition 3.5 (Compare [14, Definition 2.1]). Let $(\mathbb{Q}, \leq)$ be a strategically $(<\lambda)-$ complete forcing notion and $R^{\text {pr }}$ be a $\lambda$-sequential purity on $\mathbb{Q}$. Suppose that a model $N \prec\left(\mathcal{H}(\chi), \in,<_{\chi}^{*}\right)$ is such that $|N|=\lambda,{ }^{<\lambda} N \subseteq N$ and $\lambda, \mathbb{Q}, D, \mathcal{S}, \ldots \in N$ (but note we do not demand $R^{\mathrm{pr}} \in N$ ) and a function $h: \lambda \longrightarrow N$ is such that its range $\operatorname{rng}(h)$ includes $\mathbb{Q} \cap N$. Also, let $\overline{\mathcal{I}}=\left\langle\mathcal{I}_{\alpha}: \alpha<\lambda\right\rangle$ list all dense open subsets of $\mathbb{Q}$ belonging to $N$ and let $\gamma<\lambda$.

(1) We say that a sequence $\bar{f}=\left\langle f_{\delta}: \delta \in \mathcal{S}\right\rangle$ is a $(D, \mathcal{S}, h)$-semi diamond for $\mathbb{Q}$ over $N$ if $f_{\delta} \in{ }^{\delta} \delta$ for $\delta \in \mathcal{S}$ and 


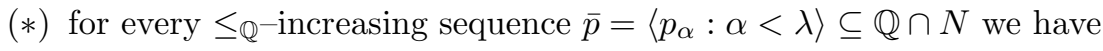

$$
\left\{\delta \in \mathcal{S}:(\forall \alpha<\delta)\left(h\left(f_{\delta}(\alpha)\right)=p_{\alpha}\right)\right\} \in D^{+}
$$

Below, let $\bar{f}$ be a $(D, \mathcal{S}, h)$-semi diamond for $\mathbb{Q}$ over $N$.

(2) An $\left(N, h, \mathbb{Q}, R^{\mathrm{pr}}, \bar{f}, \overline{\mathcal{I}}\right)$-candidate is a sequence $\bar{q}=\left\langle q_{\delta}: \delta \in \mathcal{S}\right.$ limit $\rangle$ of conditions from $N \cap \mathbb{Q}$ satisfying for each limit $\delta \in \mathcal{S}$ :

(a) if $h \circ f_{\delta}=\left\langle h\left(f_{\delta}(\alpha)\right): \alpha<\delta\right\rangle \subseteq \mathbb{Q} \cap N$ and it has an upper bound in $\mathbb{Q}$, then $h\left(f_{\delta}(\alpha)\right) \leq q_{\delta}$ for all $\alpha<\delta$, and

(b) if, moreover, $h \circ f_{\delta} \in \operatorname{dom}\left(R^{\mathrm{pr}}\right)$, then also $h \circ f_{\delta} R^{\mathrm{pr}} q_{\delta}$, and

(c) if there is $q \in \bigcap_{\alpha<\delta} \mathcal{I}_{\alpha}$ such that $h \circ f_{\delta} R^{\text {pr }} q$, then also $q_{\delta} \in \bigcap_{\alpha<\delta} \mathcal{I}_{\alpha}$.

If $N, h, \mathbb{Q}, R^{\mathrm{pr}}, \bar{f}, \overline{\mathcal{I}}$ are obvious from the context we may just say a candidate.

(3) Let $\bar{q}=\left\langle q_{\delta}: \delta \in \mathcal{S} \& \delta\right.$ is limit $\rangle$ be a candidate and $r \in \mathbb{Q}$. We define a game $\partial_{\gamma}^{\mathcal{S}}\left(r, N, h, \mathbb{Q}, R^{\mathrm{pr}}, \bar{f}, \bar{q}\right)$ of two players, Generic and Antigeneric, as follows. A play lasts $\leq \lambda$ moves and in the $i^{\text {th }}$ move the players try to choose conditions $r_{i}^{-}, r_{i} \in \mathbb{Q}$ and a set $C_{i} \in D$ so that ${ }^{3}$ :

(a) $r \leq r_{i}$, and $r_{i}^{-} \in N$, and if $i \notin \mathcal{S}[\gamma] \cap \mathcal{R}$ then $r_{i}^{-} \leq r_{i}$,

(b) $(\forall i<j<\lambda)\left(r_{i} \leq r_{j} \& r_{i}^{-} \leq r_{j}^{-}\right)$, and

(c) Generic chooses $r_{i}^{-}, r_{i}, C_{i}$ if $i \in \mathcal{S}[\gamma]$, and Antigeneric chooses $r_{i}^{-}, r_{i}, C_{i}$ if $i \notin \mathcal{S}[\gamma]$.

At the end Generic wins the play whenever both players always had legal moves (so the game lasted $\lambda$ steps) and

$(\circledast)$ if $\delta \in \mathcal{S}[\gamma] \cap \bigcap_{i<\delta} C_{i}$ is a limit ordinal and $h \circ f_{\delta}$ is an increasing sequence of conditions in $\mathbb{Q}$ such that for all $\alpha<\delta$ we have $h\left(f_{\delta}(\alpha+1)\right)=r_{\alpha+1}^{-}$, then $q_{\delta} \leq r_{\delta}$ and $h \circ f_{\delta} R^{\text {pr }} r_{\delta}$.

(4) Let $\bar{q}$ be a candidate (for $N, h, \mathbb{Q}, R^{\mathrm{pr}}, \bar{f}$ and $\overline{\mathcal{I}}$ ). A condition $r \in \mathbb{Q}$ is generic for the candidate $\bar{q}$ over $N, h, \mathbb{Q}, R^{\mathrm{pr}}, \bar{f}, \mathcal{S}, \gamma$ if Generic has a winning strategy in the game $\partial_{\gamma}^{\mathcal{S}}\left(r, N, h, \mathbb{Q}, R^{\mathrm{pr}}, \bar{f}, \bar{q}\right)$.

Definition 3.6 (Compare [14, Definition 2.3]). Let $\mathbb{Q}$ be a strategically $(<\lambda)$ complete forcing notion.

(1) We say that $\mathbb{Q}$ is purely sequentially proper over $(D, \mathcal{S})$-semi diamonds whenever the following condition $(\odot)$ is satisfied.

$(\odot)$ Assume that $\chi$ is a large enough regular cardinal and $N \prec(\mathcal{H}(\chi), \in$ ,$\left.<_{\chi}^{*}\right),|N|=\lambda,{ }^{<\lambda} N \subseteq N$ and $\lambda, \mathbb{Q}, D, \mathcal{S}, \ldots \in N$. Then there exists a $\lambda$-sequential purity $R^{\text {pr }}$ on $\mathbb{Q}$ such that for every ordinal $\gamma<\lambda$, a condition $p \in \mathbb{Q} \cap N$ and every $\overline{\mathcal{I}}, h, \bar{f}, \bar{q}$ satisfying

- $\overline{\mathcal{I}}=\left\langle\mathcal{I}_{\alpha}: \alpha<\lambda\right\rangle$ lists all open dense subsets of $\mathbb{Q}$ from $N$,

- a function $h: \lambda \longrightarrow N$ is such that $\mathbb{Q} \cap N \subseteq \operatorname{rng}(h)$, and

- a sequence $\bar{f}$ is a $(D, \mathcal{S}, h)$-semi diamond for $\mathbb{Q}$, and

- $\bar{q}$ is an $\left(N, h, \mathbb{Q}, R^{\mathrm{pr}}, \bar{f}, \overline{\mathcal{I}}\right)$-candidate,

there is a condition $r \geq p$ generic for $\bar{q}$ over $N, h, \mathbb{Q}, R^{\mathrm{pr}}, \bar{f}, \mathcal{S}, \gamma$.

(2) If in the condition $(\odot)$ of $(1)$ above

(a) the relation $R^{\mathrm{pr}}$ can be required to be a $\lambda$-sequential ${ }^{+}$purity, then we say that $\mathbb{Q}$ is purely sequentially ${ }^{+}$proper over $(D, \mathcal{S})$-semi diamonds,

\footnotetext{
${ }^{3}$ Remember Context $3.1(3,4)$
} 
(b) if the relation $R^{\mathrm{pr}}$ does not depend on $N$, then we add the adjective uniformly (so we say then uniformly purely sequentially proper etc).

Remark 3.7. The game $\partial_{\gamma}^{\mathcal{S}}\left(r, N, h, \mathbb{Q}, R^{\mathrm{pr}}, \bar{f}, \bar{q}\right)$ is very similar to the game $\partial(r, N, h, \mathbb{Q}, \bar{f}, \bar{q})$ of [14, Definition 2.1(3)]. Some of the differences in the definition of candidates are caused by the larger generality and the use of $R^{\mathrm{pr}}$. But one technical point is actually correcting an error in 14. When comparing [14, Definition 2.1 $(3)(\circledast)$ ] and Definition 3.5 here, one notices that in the former paper the condition $(\circledast)$ is "active" if the semi-diamond guessed $\left\langle r_{i}^{-}: i<\delta\right\rangle$, while here we "activate" this demand if the successor terms of the sequence were guessed correctly. In other words, current $(\circledast)$ is "active" more often. Now, in the proof of [14, Main Claim 3.10] we actually use this more often "active" version of $(\circledast)$ - on page 73 , in the paragraph between (3.14) and (3.15), we set $\underset{\sim}{j_{0}} \underset{\ominus}{\ominus}\left(\varepsilon_{\gamma}\right)=s^{-}\left(\varepsilon_{\gamma}\right)$ and not $\underset{j_{0}}{\ominus}\left(\varepsilon_{\gamma}\right)=r_{j_{0}}^{-}\left(\varepsilon_{\gamma}\right)$. This makes "guessing on coordinate $\varepsilon_{\gamma}$ " different from "guessing in $\mathbb{P}_{\zeta^{*}}$ ". To avoid the problem we make limit terms of semi-diamond guessing irrelevant. This change should be introduced to [14, Definition $2.1(3)(\circledast)$ ] and this condition should read:

$(\circledast)^{\text {corrected }}$ if $\delta \in S \cap \bigcap_{i<\delta} C_{i}$ is a limit ordinal and $h \circ F_{\delta}$ is an increasing sequence of conditions satisfying $h\left(F_{\delta}(\alpha+1)\right)=r_{\alpha+1}^{-}$for all $\alpha<\delta$, then $q_{\delta} \leq r_{\delta}$.

Then the properness over $(D, \mathcal{S})$-semi diamonds of [14, Definition 2.3] becomes a potentially stronger property, but the examples presented there still satisfy it.

Now,

$(\otimes)$ if $\mathbb{P}$ is proper over $(D, \mathcal{S}[\gamma])$-semi diamonds (see [14, Definition 2.3] plus the correction stated above) for each $\gamma<\lambda$, then $\mathbb{P}$ is uniformly purely sequentially ${ }^{+}$proper over $(D, \mathcal{S})-$ semi diamonds.

Why? First note that $\mathbb{P}$ is $(<\lambda)$-complete and define a binary relation $R^{\text {pr }}$ by:

$\bar{p} R^{\text {pr }} p$ if and only if $\bar{p}=\left\langle p_{\alpha}: \alpha<\delta\right\rangle$ is a $\leq_{\mathbb{P}}$-increasing sequence of conditions in $\mathbb{P}, \delta<\lambda$ is a limit ordinal and $p$ is an upper bound to $\bar{p}$.

Then $R^{\text {pr }}$ is a $\lambda$-sequential ${ }^{+}$purity. Suppose $N, h, \bar{f}, \overline{\mathcal{I}}, \gamma, \bar{q}=\left\langle q_{\delta}: \delta \in \mathcal{S}\right.$ is limit $\rangle$ and $p$ are as in $3.6(1)(\odot)$. For $\delta \in \mathcal{S}[\gamma]$ let $q_{\delta}^{*} \in \mathbb{P}$ be such that

if $\delta$ is limit, $h \circ f_{\delta} \subseteq \mathbb{P} \cap N$ is a $\leq_{\mathbb{P}}$-increasing sequence and $q_{\delta}$ is its upper bound, then $q_{\delta}^{*}=q_{\delta}$,

otherwise $q_{\delta}^{*}$ is the $<_{\chi}^{*}$-first member of $\bigcap_{i<\delta} \mathcal{I}_{i}$.

Plainly, $\bar{q}^{*}=\left\langle q_{\delta}^{*}: \delta \in \mathcal{S}[\gamma]\right\rangle$ is an $(N, h, \mathbb{P})$-candidate over $\bar{f}$ in the sense of [14, Definition 2.1(2)] (and if $\delta \in \mathcal{S}[\gamma]$ is limit and $h \circ f_{\delta}$ is an increasing sequence of conditions from $\mathbb{P}$, then $q_{\delta}^{*}=q_{\delta}$ ). Let st be a winning strategy of Generic in the game $\partial\left(r, N, h, \mathbb{P}, \bar{f}, \bar{q}^{*}\right)$ of [14, Definition 2.1]. The same strategy can be used by Generic in $\partial_{\gamma}^{\mathcal{S}}\left(r, N, h, \mathbb{P}, R^{\mathrm{pr}}, \bar{f}, \bar{q}\right)$ and easily it is a winning strategy in this game too.

Proposition 3.8. Let $\mathbb{Q}, N, h, \overline{\mathcal{I}}, R^{\mathrm{pr}}, \gamma$ be as in Definition 3.5.

(1) Every $(D, \mathcal{S})$-diamond is a $(D, \mathcal{S}, h)$-semi diamond for $\mathbb{Q}$ over $N$. Below, let $\bar{f}$ be a $(D, \mathcal{S}, h)$-semi diamond for $\mathbb{Q}$ over $N$.

(2) There exists an $\left(N, h, \mathbb{Q}, R^{\mathrm{pr}}, \bar{f}, \overline{\mathcal{I}}\right)$-candidate. Below, let $\bar{q}$ be an $\left(N, h, \mathbb{Q}, R^{\mathrm{pr}}, \bar{f}, \overline{\mathcal{I}}\right)$-candidate. 
(3) If $\mathbb{Q}$ is $(<\lambda)$-lub-complet $\Theta^{\mathbb{4}}$, then for any condition $r \in \mathbb{Q}$ both players have always legal moves in the game $\partial_{\gamma}^{\mathcal{S}}\left(r, N, h, \mathbb{Q}, R^{\mathrm{pr}}, \bar{f}, \bar{q}\right)$ satisfying $r_{j}^{-} \leq r_{j}$, provided that for each $i \in \mathcal{S}[\gamma] \cap \mathcal{R} \cap j$ conditions $r_{i}^{-}$, $r_{i}$ are compatible.

(4) If $\mathbb{Q}$ is $(<\lambda)$-complete and $r \in \mathbb{Q}$ is $(N, \mathbb{Q})$-generic in the standard sense, then also both players have always legal moves in the game $\partial_{\gamma}^{\mathcal{S}}\left(r, N, h, \mathbb{Q}, R^{\mathrm{pr}}, \bar{f}, \bar{q}\right)$ satisfying $r_{j}^{-} \leq r_{j}$, provided that for each $i \in \mathcal{S}[\gamma] \cap \mathcal{R} \cap j$ conditions $r_{i}^{-}, r_{i}$ are compatible.

(5) If $r \in \mathbb{Q}$ is generic for $\bar{q}$ over $N, h, \mathbb{Q}, R^{\mathrm{pr}}, \bar{f}, \mathcal{S}, \gamma$, then $r$ is $(N, \mathbb{Q})$-generic in the standard sense.

(6) Consequently, if a forcing notion $\mathbb{Q}$ is purely sequentially proper over $(D, \mathcal{S})$ semi diamonds and there exists a $(D, \mathcal{S})$-diamond, then $\mathbb{Q}$ is $\lambda$-proper in the standard sense.

Proof. (5) $\quad$ Let $\bar{q}$ be an $\left(N, h, \mathbb{Q}, R^{\mathrm{pr}}, \bar{f}, \overline{\mathcal{I}}\right)$-candidate and $r \in \mathbb{Q}$ be generic for $\bar{q}$ over $N, h, \mathbb{Q}, R^{\operatorname{pr}}, \bar{f}, \mathcal{S}, \gamma$. Suppose that $\mathcal{I} \in N$ is an open dense subset of $\mathbb{Q}$, say $\mathcal{I}=\mathcal{I}_{j_{0}}$ (remember, $\overline{\mathcal{I}}=\left\langle\mathcal{I}_{i}: i<\lambda\right\rangle$ lists all open dense subsets of $\mathbb{Q}$ belonging to $N)$. We want to argue that $\mathcal{I} \cap N$ is predense above $r$, so suppose $r_{0} \geq r$.

Consider a play of $\partial_{\gamma}^{\mathcal{S}}\left(r, N, h, \mathbb{Q}, R^{\mathrm{pr}}, \bar{f}, \bar{q}\right)$ in which Generic follows her winning strategy and Antigeneric plays as follows.

- At stage $i=0$, Antigeneric sets $C_{0}=\lambda, r_{0}^{-}=\emptyset_{\mathbb{Q}}$ and $r_{0}$ is the one fixed above. - At a stage $i \notin \mathcal{S}[\gamma], i>0$, Antigeneric first picks any legal mov $\int^{5} C_{i}, r_{i}^{-}, r_{i}^{\prime}$ and then "corrects" it by choosing a condition $r_{i} \geq r_{i}^{\prime}$ so that $r_{i} \in \bigcap_{j<i} \mathcal{I}_{j}$ (remember, $\mathbb{Q}$ is strategically $(<\lambda)$-complete).

After the play is completed and a sequence $\left\langle C_{i}, r_{i}^{-}, r_{i}: i\langle\lambda\rangle\right.$ is constructed, we know that the condition $(\circledast)$ of Definition 3.5 (3) is satisfied. Also, since $\bar{f}$ is a $(D, \mathcal{S}, h)$-semi diamond for $\mathbb{Q}$ over $N$, we know that $\left\{\delta \in \mathcal{S}:(\forall \alpha<\delta)\left(h\left(f_{\delta}(\alpha)\right)=\right.\right.$ $\left.\left.r_{\alpha}^{-}\right)\right\} \in D^{+}$. Pick a limit ordinal $\delta \in \mathcal{S}[\gamma] \cap \triangle C_{i<\lambda}$ such that $\delta>j_{0}, \delta$ is a limit of elements of $\lambda \backslash \mathcal{S}$ and $h \circ f_{\delta}=\left\langle r_{\alpha}^{-}: \alpha<\delta\right\rangle$. Then by $(\circledast)$ we have that $q_{\delta} \leq r_{\delta}$ and $h \circ f_{\delta} R^{\mathrm{pr}} r_{\delta}$. Moreover, since $r_{\alpha} \leq r_{\delta}$ for all $\alpha<\delta$ and since $\delta$ is a limit of points from $\lambda \backslash \mathcal{S}$ we get $r_{\delta} \in \bigcap_{j<\delta} \mathcal{I}_{j}$. Therefore (by 3.5 (2)(c)) $q_{\delta} \in \bigcap_{j<\delta} \mathcal{I}_{j}$, so in particular $q_{\delta} \in \mathcal{I}_{j_{0}} \cap N$. But the condition $r_{\delta}$ is stronger than $q_{\delta}$ and it is also stronger than $r_{0}$, so $r_{0}$ is compatible with $q_{\delta}$.

Proposition 3.9. Assume that a forcing notion $\mathbb{P}$ is one of the following types:

(a) $\mathbb{P}=\mathbb{S}_{E}^{\psi}$ where $\psi, E$ are as in Definition 2.22(1), and

$$
A \in E \Rightarrow(\lambda \backslash \mathcal{S}) \cup A \in D,
$$

(b) $\mathbb{P}=\mathbb{Q}^{\ell, \bar{E}}$ where $\bar{E}$ is as in Definition 2.18 and $1<\ell \leq 4$, or

(c) $\mathbb{P}=\mathbb{Q}_{E}^{3, \bar{E}}$ where $\bar{E}, E$ are as in Definition 2.18 , and

$$
A \in E \Rightarrow(\lambda \backslash \mathcal{S}) \cup A \in D,
$$

(d) $\mathbb{P}=\mathbb{Q}_{\varphi, \bar{F}}^{\ell}$ where $\lambda, \varphi, \bar{F}$ are as in Definition 2.20, $1<\ell \leq 4$, or

(e) $\mathbb{P}=\mathbb{Q}_{\varphi, \bar{F}, E}^{3}$ where $\bar{E}, E$ are as in Definition [2.20, and

$$
A \in E \Rightarrow(\lambda \backslash \mathcal{S}) \cup A \in D,
$$

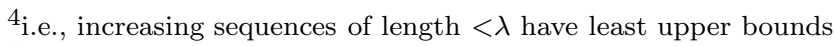

${ }^{5}$ Note that since Generic uses her winning strategy, there are always legal moves.
} 
(f) $\mathbb{P}=\mathbb{S}_{E}^{\psi}$ where $\psi, E$ are as in Definition 2.22, and $\lambda \backslash \mathcal{S} \in E$, or

(g) $\mathbb{P}=\mathbb{Q}_{E}^{\ell, \bar{E}}$ or $\mathbb{P}=\mathbb{Q}_{\varphi, \bar{F}, E}^{\ell}$, where $\ell=1$ or $\ell=3, \bar{E}, E$ are as in Definition 2.18 or $\lambda, \varphi, \bar{F}, E$ are as in Definition 2.20, respectively, and $\lambda \backslash \mathcal{S} \in E$, or

(h) $\mathbb{P}=\mathbb{H}_{\lambda}$ or $\mathbb{P}=\mathbb{E}_{\lambda}$ (see Definition 2.22(2,3)),

(i) $\mathbb{P}$ is strategically $(\leq \lambda)$-complete and $(<\lambda)$-complete.

Then the forcing notion $\mathbb{P}$ is uniformly purely sequentially ${ }^{+}$proper over $(D, \mathcal{S})-$ semi diamonds.

Proof. (a) The arguments here are very similar to those for $\mathbb{Q}_{\varphi, \bar{F}, E}^{3}$ below, but much simpler. We will use the fact that the forcing notion $\mathbb{S}_{E}^{\psi}$ is $(<)$-lub-complete without explicitly saying this. Define a binary relation $R^{\mathrm{pr}}$ by:

$\bar{p} R^{\mathrm{pr}} p$ if and only if

- $\bar{p}=\left\langle p_{\alpha}: \alpha<\delta\right\rangle$ is an increasing sequence of conditions from $\mathbb{S}_{E}^{\psi}$ such that $\delta<\lambda$ is a limit ordinal, and

- $p \in \mathbb{S}_{E}^{\psi}$ is an upper bound of the sequence $\bar{p}$ with $\operatorname{rt}(p)=\operatorname{rt}\left(\bigcup_{\alpha<\delta} p_{\alpha}\right)$.

Plainly, $R^{\text {pr }}$ is a $\lambda$-sequential ${ }^{+}$purity on $\mathbb{S}_{E}^{\psi}$. Now, let $N, h, \bar{f}, \overline{\mathcal{I}}, \gamma$ be as in the assumptions of $3.6(1)(\odot)$ and let $\bar{q}=\left\langle q_{\delta}: \delta \in \mathcal{S}\right.$ is limit $\rangle$ be an appropriate candidate. Suppose $p \in \mathbb{S}_{E}^{\psi} \cap N$. We will define a condition $p_{\lambda} \geq p$ generic for $\bar{q}$. For this, by induction on $\alpha<\lambda$ we choose conditions $p_{\alpha} \in \mathbb{S}_{E}^{\psi} \cap N$ as follows.

$(\boldsymbol{\Delta})_{1} p_{0}=p$ and if $\delta$ is a limit ordinal, then $p_{\delta}=\bigcup_{\xi<\delta} p_{\xi}$.

$(\boldsymbol{\Delta})_{2}$ Suppose that $\alpha=\delta+1, \delta \in \mathcal{S}[\gamma]$ is a limit ordinal, and $h \circ f_{\delta}$ is an increasing sequence of conditions from $\mathbb{S}_{E}^{\psi} \cap N$ such that $p_{\delta} \leq \bigcup_{\beta<\delta} h\left(f_{\delta}(\beta)\right)$. Then we let $p_{\alpha}=\left(p_{\delta}\lceil\alpha) \cup\left(q_{\delta}\lceil[\alpha, \lambda))\right.\right.$.

$(\boldsymbol{\Delta})_{3}$ If $\alpha=\delta+1$ and the clause $(\boldsymbol{\Delta})_{2}$ does not apply, then $p_{\alpha}=p_{\delta}$.

Under the assumptions of $(\boldsymbol{\Delta})_{2}$ we have $p_{\delta} \leq \bigcup_{\beta<\delta} h\left(f_{\delta}(\beta)\right) \leq q_{\delta}$. Therefore, it should be clear that the construction can be carried out and that the resulting sequence $\left\langle p_{\alpha}: \alpha<\lambda\right\rangle$ satisfies the assumptions of Lemma 2.25. Consequently $p_{\lambda} \stackrel{\text { def }}{=} \bigcup_{\alpha<\lambda} p_{\alpha} \in \mathbb{S}_{E}^{\psi}$

Let us show that $p_{\lambda}$ is generic for $\bar{q}$ over $N, h, \mathbb{S}_{E}^{\psi}, R^{\mathrm{pr}}, \bar{f}, \mathcal{S}, \gamma$. Consider the following strategy st of Generic in the game $\partial_{\gamma}^{\mathcal{S}}\left(p_{\lambda}, N, h, \mathbb{S}_{E}^{\psi}, R^{\mathrm{pr}}, \bar{f}, \bar{q}\right)$. At a stage $i \in \mathcal{S}[\gamma]$, when a sequence $\left\langle r_{j}^{-}, r_{j}, C_{j}: j<i\right\rangle$ has been already constructed, Generic picks $r_{i}^{-}, r_{i}, C_{i}$ so that

$(\boldsymbol{\Delta})_{4}$ if $i$ is limit, then $r_{i}^{-}=\bigcup_{j<i} r_{j}^{-}, r_{i}=\bigcup_{j<i} r_{j}$ and $C_{i}=\bigcap_{j<i} C_{j}$,

$(\boldsymbol{\Delta})_{5}$ if $i$ is a successor, say $i=j+1$, then $r_{i}^{-}, r_{i} \in \mathbb{S}_{E}^{\psi}$ are chosen so that $r_{j} \leq r_{i}, \operatorname{rt}\left(r_{j}\right)<\operatorname{rt}\left(r_{i}^{-}\right)=\operatorname{rt}\left(r_{i}\right), r_{j}^{-} \leq r_{i}^{-}, p_{i} \leq r_{i}^{-}$and $r_{i}^{-} \leq r_{i}$. Also, $C_{i}=\lambda \backslash\left(\operatorname{dom}\left(r_{i}\right) \cap \mathcal{S}\right)$.

Note that at a stage $i=j+1$, since $r_{j}^{-} \leq r_{j}$ and $p_{i} \leq p_{\lambda} \leq r_{j}$, we know that $r_{j}^{-} \cup p_{i} \in \mathbb{S}_{E}^{\psi} \cap N$ and $\left(r_{j}^{-} \cup p_{i}\right) \leq r_{j}$. Therefore Generic may easily pick $r_{i}^{-}, r_{i}$ as required. Also, by our assumption on $E$, we are sure that $C_{i} \in D$.

Suppose now that $\left\langle r_{i}^{-}, r_{i}, C_{i}: i<\lambda\right\rangle$ is a play of $\partial_{\gamma}^{\mathcal{S}}\left(T^{*}, N, h, \mathbb{S}_{E}^{\psi}, R^{\mathrm{pr}}, \bar{f}, \bar{q}\right)$ in which Generic follows the strategy st described above. Let $\delta \in \mathcal{S}[\gamma] \cap \bigcap_{i<\delta} C_{i}$ 
be a limit ordinal such that $h \circ f_{\delta}$ is an increasing sequence of conditions from $\mathbb{S}_{E}^{\psi}$ satisfying $h\left(f_{\delta}(i+1)\right)=r_{i+1}^{-}$for all $i<\delta$. By $(\mathbf{\Lambda})_{5}+(\mathbf{\Lambda})_{4}$ we have that $i \leq \operatorname{rt}\left(h\left(f_{\delta}(i)\right)\right)<\delta$ and $p_{i} \leq_{\mathbb{S}_{E}^{\psi}} h\left(f_{\delta}(i)\right)$ (for all successor $i<\delta$ ) and $\delta \notin$ $\operatorname{dom}\left(\bigcup_{j<\delta} r_{j}^{-}\right)=\operatorname{dom}\left(\bigcup_{j<\delta} h\left(f_{\delta}(j)\right)\right.$. Therefore $q_{\delta}\left\lceil[\delta+1, \lambda) \subseteq p_{\delta+1} \subseteq p_{\lambda} \subseteq r_{\delta}\right.$, $q_{\delta}\left\lceil\delta=\left(\bigcup_{j<i} h\left(f_{\delta}(j)\right)\left\lceil\delta=r_{\delta}\left\lceil\delta\right.\right.\right.\right.$ and $\delta=\operatorname{rt}\left(r_{\delta}\right)=\operatorname{rt}\left(q_{\delta}\right)=\operatorname{rt}\left(\bigcup_{j<i} h\left(f_{\delta}(j)\right)\right)$. Consequently, $q_{\delta} \leq r_{\delta}$ and $h \circ f_{\delta} R^{\mathrm{pr}} r_{\delta}$.

(b,c,d,e) We know that the forcing notion $\mathbb{P}$ is strategically $(<\lambda)$-complete (see Observation 2.23). Define a binary relation $R^{\mathrm{pr}}$ by:

$\bar{T} R^{\mathrm{pr}} T$ if and only if

- $\bar{T}=\left\langle T_{\alpha}: \alpha<\delta\right\rangle$ is a $\leq \mathbb{P}^{-}$-increasing sequence of conditions from $\mathbb{P}$ such that $\delta<\lambda$ is a limit ordinal and for $\alpha<\delta$ we have $\alpha \leq \operatorname{lh}\left(\operatorname{root}\left(T_{\alpha}\right)\right)<\delta$, and

- $T \in \mathbb{P}$ is a $\leq_{\mathbb{P}^{-}}$upper bound of the sequence $\bar{T}$ with $\operatorname{root}(T)=\operatorname{root}\left(\bigcap_{\alpha<\delta} T_{\alpha}\right)$. (Note that the previous bullet implies $\bigcap T_{\alpha} \in \mathbb{P}$. In cases (b) and (d) we also know that $\bigcup_{\alpha<\delta} \operatorname{root}\left(T_{\alpha}\right)=\operatorname{root}\left(\bigcap_{\alpha<\delta}^{\alpha<\delta} T_{\alpha}\right)$.)

Easily, $R^{\text {pr }}$ is a $\lambda$-sequential ${ }^{+}$purity on $\mathbb{P}$. Suppose that $N \prec\left(\mathcal{H}(\chi), \in,<_{\chi}^{*}\right)$ and $h, \bar{f}, \overline{\mathcal{I}}, \gamma$ are as in the assumptions of Condition $\underline{3.6}(1)(\odot)$. Let $\bar{q}=\left\langle q_{\delta}: \delta \in\right.$ $\mathcal{S}$ is limit $\rangle$ be an $\left(N, h, \mathbb{P}, R^{\text {pr }}, \bar{f}, \overline{\mathcal{I}}\right)$-candidate and let $T \in \mathbb{P} \cap N$. We want to find a condition $T^{*} \geq T$ generic for $\bar{q}$ over $N, h, \mathbb{P}, R^{\mathrm{pr}}, \bar{f}, \mathcal{S}, \gamma$. To this end we choose by induction on $\alpha<\lambda$ conditions $T_{\alpha} \in \mathbb{P} \cap N$ as follows.

$(\triangle)_{1} T_{0}=T$ and if $\delta$ is a limit ordinal, then $T_{\delta}=\bigcap_{\xi<\delta} T_{\xi}$.

$(\triangle)_{2}$ Suppose that $\alpha=\delta+1, \delta \in \mathcal{S}[\gamma]$ is a limit ordinal, and $h \circ f_{\delta}$ is a $\leq_{\mathbb{P}^{-}}$ increasing sequence of conditions from $\mathbb{P} \cap N$ such that for all successor $\beta<\delta$ we have

$$
\beta \leq \operatorname{lh}\left(\operatorname{root}\left(h\left(f_{\delta}(\beta)\right)\right)\right)<\delta \text { and } T_{\beta} \leq h\left(f_{\delta}(\beta)\right) .
$$

Let $T_{\alpha}^{*}=\left\{t \in T_{\delta}: \operatorname{root}\left(\bigcap_{\beta<\delta} h\left(f_{\delta}(\beta)\right)\right) \unlhd t \Rightarrow t \in q_{\delta}\right\}$. If $\ell \neq 3$ then $T_{\alpha}^{*} \in \mathbb{P}$ and we put $T_{\alpha}=T_{\alpha}^{*}$. Otherwise, we choose $T_{\alpha} \in \mathbb{P}$ such that $T_{\alpha} \subseteq T_{\alpha}^{*}$ and $T_{\alpha} \cap{ }^{\alpha} \lambda=T_{\alpha}^{*} \cap{ }^{\alpha} \lambda$.

$(\triangle)_{3}$ If $\alpha=\delta+1$ and the clause $(\triangle)_{2}$ does not apply, then $T_{\alpha}=T_{\delta}$.

Suppose that $\alpha=\delta+1$ satisfies the assumptions of $(\triangle)_{2}$. Then $\bigcap_{\beta<\delta} h\left(f_{\delta}(\beta)\right) \in \mathbb{P}$ (remember Observation 2.23) and $T_{\delta} \leq \bigcap_{\beta<\delta} h\left(f_{\delta}(\beta)\right) \leq q_{\delta}$ and $\operatorname{root}\left(q_{\delta}\right)=\operatorname{root}\left(\bigcap_{\beta<\delta} h\left(f_{\delta}(\beta)\right)\right) \in$ $T_{\delta}$. Let $t_{\delta}=\operatorname{root}\left(\bigcap_{\beta<\delta} h\left(f_{\delta}(\beta)\right)\right)$. If $\operatorname{lh}\left(t_{\delta}\right)=\delta$, then also $t_{\delta}=\bigcup_{\beta<\delta} \operatorname{root}\left(h\left(f_{\delta}(\beta)\right)\right)$ and $\operatorname{succ}_{T_{\delta+1}}\left(t_{\delta}\right)=\operatorname{succ}_{q_{\delta}}\left(t_{\delta}\right)$. (Note that in cases (b) and (d) this must happen.) If $\operatorname{lh}\left(t_{\delta}\right)>\delta$, then $\bigcup_{\beta<\delta} \operatorname{root}\left(h\left(f_{\delta}(\beta)\right)\right)=t_{\delta}\left\lceil\delta \triangleleft t_{\delta}\right.$ and $\operatorname{succ}_{T_{\delta+1}}\left(t_{\delta}\lceil\delta)=\operatorname{succ}_{T_{\delta}}\left(t_{\delta}\lceil\delta)\right.\right.$. (This may happen only in cases (c) and (e).) 
Therefore we may conclude that the sequence $\left\langle T_{\alpha}: \alpha<\lambda\right\rangle$ satisfies the assumptions of Lemma 2.24. At limit stages $\delta<\lambda$ this implies that $T_{\delta}=\bigcap_{\beta<\delta} T_{\beta} \in \mathbb{P}$ (so the construction can be carried out) and for $\lambda$ we get $T^{*} \stackrel{\text { def }}{=} \bigcap_{\alpha<\lambda} T_{\alpha} \in \mathbb{P}$.

Claim 3.9.1. $T^{*}$ is $(N, \mathbb{P})$-generic in the standard sense.

Proof of the Claim. Suppose towards contradiction that there are $\xi_{0}<\lambda$ and a condition $T^{+} \geq T^{*}$ such that $T^{+}$is incompatible with every member of $\mathcal{I}_{\xi_{0}} \cap N$. Construct inductively a sequence $\left\langle S_{\alpha}: \alpha<\lambda\right\rangle$ of conditions in $\mathbb{P}$ such that

- $T^{+} \leq S_{\beta} \leq S_{\alpha}, \operatorname{lh}\left(\operatorname{root}\left(S_{\beta}\right)\right)<\operatorname{lh}\left(\operatorname{root}\left(S_{\alpha}\right)\right)$ for $\beta<\alpha<\lambda$, and

- $S_{\alpha+1} \in \bigcap_{\xi \leq \alpha} \mathcal{I}_{\xi}$ and if $\alpha$ is limit, then $S_{\alpha}=\bigcap_{\beta<\alpha} S_{\beta}$ (for all $\alpha<\lambda$ ).

Let $t_{\alpha}=\operatorname{root}\left(S_{\alpha}\right)$. Since $S_{\alpha} \subseteq T^{+} \subseteq T^{*} \subseteq T_{\alpha}$ we see that $t_{\alpha} \in T_{\alpha}$ and $\left(T_{\alpha}\right)_{t_{\alpha}} \leq S_{\alpha}$. Now we claim that

$(\nabla)$ there is a limit ordinal $\delta \in \mathcal{S}[\gamma]$ such that $\xi_{0}<\delta$ and

$$
h \circ f_{\delta}=\left\langle\left(T_{\alpha}\right)_{t_{\alpha}}: \alpha<\delta\right\rangle, \quad t_{\delta}=\bigcup_{\alpha<\delta} t_{\alpha}, \quad \operatorname{lh}\left(t_{\delta}\right)=\delta \text { and } S_{\delta} \in \bigcap_{\alpha<\delta} \mathcal{I}_{\alpha} .
$$

This should be clear in cases (b) and (d) (as closed unbounded subsets of $\lambda$ belong to $D)$. In cases (c) and (e) we note that

$$
A:=\left\{\delta \in \mathcal{S}[\gamma]: \delta \text { limit and } h \circ f_{\delta}=\left\langle\left(T_{\alpha}\right)_{t_{\alpha}}: \alpha<\delta\right\rangle\right\} \in D^{+} .
$$

Letting $\eta=\bigcup_{\alpha<\lambda} t_{\alpha}$ we have $\eta \in \lim _{\lambda}\left(S_{\alpha}\right)$, so

$$
A_{\alpha}:=\left\{\delta<\lambda: \mid \operatorname{succ}_{S_{\alpha}}(\eta\lceil\delta) \mid>1\} \in E\right.
$$

(for each $\alpha<\lambda$ ). By our additional assumptions on $E$ we conclude that

$$
A \cap \triangle_{\alpha<\lambda} A_{\alpha} \cap\left\{\delta \in \mathcal{S}[\gamma] \backslash\left(\xi_{0}+1\right):(\forall \alpha<\delta)\left(\ln \left(t_{\alpha}\right)<\delta\right)\right\} \neq \emptyset
$$

Then any $\delta$ from the set above will witness $(\varnothing)$.

So let $\delta$ be as given by $(\nabla)$. Then $h \circ f_{\delta} R^{\mathrm{pr}} S_{\delta}$ so by 3.5 (2) we conclude $q_{\delta} \in \bigcap_{\alpha<\delta} \mathcal{I}_{\alpha}$ (in particular $\left.q_{\delta} \in \mathcal{I}_{\xi_{0}} \cap N\right)$. But $q_{\delta} \leq\left(T_{\delta+1}\right)_{t_{\delta}} \leq\left(T^{*}\right)_{t_{\delta}} \leq\left(T^{+}\right)_{t_{\delta}}$, contradicting the choice of $T^{+}$.

Now we are ready to argue that $T^{*}$ is generic for $\bar{q}$ over $N, h, \mathbb{P}, R^{\text {pr }}, \bar{f}, \mathcal{S}, \gamma$ and for this let us consider the following strategy st of Generic in the game $\partial_{\gamma}^{\mathcal{S}}\left(T^{*}, N, h, \mathbb{P}, R^{\mathrm{pr}}, \bar{f}, \bar{q}\right)$. At a stage $i \in \mathcal{S}[\gamma]$, when a sequence $\left\langle r_{j}^{-}, r_{j}, C_{j}: j<i\right\rangle$ has been already constructed, Generic picks $r_{i}^{-}, r_{i}, C_{i}$ so that

$(\triangle)_{4}$ if $i$ is limit, then $r_{i}^{-}=\bigcap_{j<i} r_{j}^{-}, r_{i}=\bigcap_{j<i} r_{j}$ and $C_{i}=\bigcap_{j<i} C_{j}$,

$(\triangle)_{5}$ if $i$ is a successor, say $i=j+1$, then $r_{i}^{-}, r_{i}$ are chosen so that $r_{j} \leq r_{i}$, $\operatorname{root}\left(r_{j}\right) \triangleleft \operatorname{root}\left(r_{i}^{-}\right)=\operatorname{root}\left(r_{i}\right), r_{j}^{-} \leq r_{i}^{-}, T_{i} \leq r_{i}^{-}$and $r_{i}^{-} \leq r_{i}$. Also, $C_{i}=\left[\operatorname{lh}\left(\operatorname{root}\left(r_{i}\right)\right)+1001, \lambda\right)$ in cases (b), (d) and $C_{i}=(\lambda \backslash \mathcal{S}) \cup\{\alpha<\lambda$ : $\left.\left(\exists t \in r_{i}\right)\left(\operatorname{lh}(t)=\alpha \wedge\left|\operatorname{succ}_{r_{i}}(t)\right|>1\right)\right\}$ in cases $(\mathrm{c}),(\mathrm{e})$.

Why are the choices possible? By $(\triangle)_{5}$ at previous stages, for each limit $i<\lambda$ the intersection of conditions played so far is in $\mathbb{P}$ (remember Observation 2.23). Note also that then $r_{i}^{-} \leq r_{i}$. At a stage $i=j+1$, since $r_{j}^{-} \leq r_{j}, T_{i} \leq T^{*} \leq r_{j}$ and $T^{*}$ is $(N, \mathbb{P})$-generic, we may find $r^{i} \in \mathbb{P} \cap N$ stronger than both $r_{j}^{-}$and $T_{i}$ and 
compatible with $r_{j}$. Then we may choose $r_{i}^{*}$ stronger than both $r^{i}$ and $r_{j}$. Pick $t \in r_{i}^{*}$ such that $\left|\operatorname{succ}_{r_{i}^{*}}(t)\right|>1$ and $\operatorname{lh}(t)>\operatorname{lh}\left(\operatorname{root}\left(r^{i}\right)\right)$ and set $r_{i}^{-}=\left(r^{i}\right)_{t}$ and $r_{i}=\left(r_{i}^{*}\right)_{t}$.

Note also that there are always legal moves for Antigeneric (use Observation 2.23 and the choices in $\left.(\triangle)_{5}\right)$.

Now suppose that $\left\langle r_{i}^{-}, r_{i}, C_{i}: i<\lambda\right\rangle$ is a play of $\partial_{\gamma}^{\mathcal{S}}\left(T^{*}, N, h, \mathbb{P}, R^{\mathrm{pr}}, \bar{f}, \bar{q}\right)$ in which Generic follows the strategy st described above. Let $\delta \in \mathcal{S}[\gamma] \cap \bigcap_{i<\delta} C_{i}$ be a limit ordinal such that $h \circ f_{\delta}$ is an increasing sequence of conditions in $\mathbb{P}$ satisfying

$$
h\left(f_{\delta}(i)\right)=r_{i}^{-} \quad \text { for all successor } i<\delta .
$$

Then our choices in $(\triangle)_{5}+(\triangle)_{4}$ imply that $i \leq \operatorname{lh}\left(\operatorname{root}\left(h\left(f_{\delta}(i)\right)\right)\right)<\delta$ and $h\left(f_{\delta}(i)\right) \geq T_{i}$ (for all successor $i<\delta$ ). Let $t=\bigcup_{i<\delta} \operatorname{root}\left(r_{i}^{-}\right)$. Since $\operatorname{lh}(t)=\delta \in C_{i}$ for all $i<\delta$, we know that $\left|\operatorname{succ}_{r_{i}}(t)\right|>1$ for each $i<\delta$ and therefore $t=$ $\operatorname{root}\left(\bigcap_{i<\delta} r_{i}\right)=\operatorname{root}\left(\bigcap_{i<\delta} r_{i}^{-}\right)$. Now using $(\triangle)_{2}+(\triangle)_{4}$ we conclude $q_{\delta}=\left(T_{\delta+1}\right)_{t} \leq$ $\left(T^{*}\right)_{t} \leq\left(r_{\delta}\right)_{t}=r_{\delta}$ and $h \circ f_{\delta} R^{\mathrm{pr}} r_{\delta}$.

(f) Like in the proof of (a) above we note that $\mathbb{S}_{E}^{\psi}$ is $(<\lambda)$-lub-complete and we will use this fact several times (without saying this). We define a binary relation $R^{\mathrm{pr}}$ by:

$\bar{p} R^{\mathrm{pr}} p \quad$ if and only if

- $\bar{p}=\left\langle p_{\alpha}: \alpha<\delta\right\rangle \subseteq \mathbb{S}_{E}^{\psi}$ is an increasing sequence of conditions such that $\delta<\lambda$ is a limit ordinal, and

- $p \in \mathbb{S}_{E}^{\psi}$ is an upper bound of the sequence $\bar{p}$.

Then $R^{\text {pr }}$ is a $\lambda$-sequential ${ }^{+}$purity on $\mathbb{S}_{E}^{\psi}$. Let $N, h, \bar{f}, \overline{\mathcal{I}}, \gamma$ be as in the assumptions of Condition $\underline{3.6}(1)(\odot)$ and $\bar{q}$ be a candidate. Suppose $p \in \mathbb{S}_{E}^{\psi} \cap N$. Choose inductively conditions $p_{\alpha} \in \mathbb{S}_{E}^{\psi} \cap N$ (for $\alpha<\lambda$ ) so that:

$(\mathbf{\nabla})_{1} p_{0} \geq p$ satisfies $\mathcal{S} \subseteq \operatorname{dom}\left(p_{0}\right)$ and if $\delta$ is limit, then $p_{\delta}=\bigcup_{\xi<\delta} p_{\xi}$.

$(\mathbf{v})_{2}$ If $\alpha=\delta+1, \delta \in \mathcal{S}[\gamma]$ is a limit ordinal, and $h \circ f_{\delta}$ is an increasing sequence of conditions from $\mathbb{S}_{E}^{\psi} \cap N$ such that $p_{\delta} \leq \bigcup_{\beta<\delta} h\left(f_{\delta}(\beta)\right)$, then $p_{\alpha}=\left(p_{\delta}\lceil\alpha) \cup\right.$ $\left(q_{\delta} \uparrow[\alpha, \lambda)\right)$.

$(\boldsymbol{\nabla})_{3}$ If $\alpha=\delta+1$ and the clause $(\mathbf{\nabla})_{2}$ does not apply, then $p_{\alpha}=p_{\delta}$.

It should be clear that the construction can be completed. We note that if $\alpha=\delta+1$ satisfies the assumptions of $(\boldsymbol{\nabla})_{2}$, then (by the choice of $\left.p_{0}\right) \delta \in \operatorname{dom}\left(p_{\delta}\right)$. Clearly the sequence $\left\langle p_{\alpha}: \alpha<\lambda\right\rangle$ satisfies the assumptions of Lemma 2.25 and hence $p_{\lambda} \stackrel{\text { def }}{=} \bigcup_{\alpha<\lambda} p_{\alpha} \in \mathbb{S}_{E}^{\psi}$.

We claim that $p_{\lambda}$ is generic for $\bar{q}$. To see this, consider the following strategy st for Generic in the game $\partial_{\gamma}^{\mathcal{S}}\left(T^{*}, N, h, \mathbb{S}_{E}^{\psi}, R^{\text {pr }}, \bar{f}, \bar{q}\right)$. At a stage $i \in \mathcal{S}[\gamma]$, when a sequence $\left\langle r_{j}^{-}, r_{j}, C_{j}: j<i\right\rangle$ has been already constructed, Generic picks $r_{i}^{-}, r_{i}, C_{i}$ so that

$(\mathbf{\nabla})_{4}$ if $i$ is limit, then $r_{i}^{-}=\bigcup_{j<i} r_{j}^{-}, r_{i}=\bigcup_{j<i} r_{j}$ and $C_{i}=\bigcap_{j<i} C_{j}$,

$(\mathbf{\nabla})_{5}$ if $i$ is a successor, say $i=j+1$, then $r_{i}^{-}, r_{i}$ are chosen so that $r_{j} \leq r_{i}$, $\operatorname{rt}\left(r_{j}\right)<\operatorname{rt}\left(r_{i}^{-}\right), r_{j}^{-} \leq r_{i}^{-}$, and $p_{i} \leq r_{i}^{-} \leq r_{i}$. Also, $C_{i}=\left[\operatorname{rt}\left(r_{i}\right)+1001, \lambda\right)$. 
Suppose now that $\left\langle r_{i}^{-}, r_{i}, C_{i}: i<\lambda\right\rangle$ is a play of $\partial_{\gamma}^{\mathcal{S}}\left(T^{*}, N, h, \mathbb{S}_{E}^{\psi}, R^{\text {pr }}, \bar{f}, \bar{q}\right)$ in which Generic follows the strategy st. Let $\delta \in \mathcal{S}[\gamma] \cap \bigcap_{i<\delta} C_{i}$ be a limit ordinal such that $h \circ f_{\delta}$ is an increasing sequence of conditions in $\mathbb{S}_{E}^{\psi}$ satisfying $h\left(f_{\delta}(i+1)\right)=r_{i+1}^{-}$ for all $i<\delta$. Then, by the description of st, we have that $i \leq \operatorname{rt}\left(h\left(f_{\delta}(i)\right)\right)<\delta$ and $p_{i} \leq h\left(f_{\delta}(i)\right)$ (for all successor $i<\delta$ ). Therefore $q_{\delta}\left\lceil[\delta+1, \lambda) \subseteq p_{\delta+1} \subseteq p_{\lambda} \subseteq r_{\delta}\right.$ and $q_{\delta}\left\lceil(\delta+1)=r_{\delta} \uparrow(\delta+1)\right.$. Consequently, $q_{\delta} \leq r_{\delta}$ and clearly $h \circ f_{\delta} R^{\text {pr }} r_{\delta}$.

(g) The forcing notion $\mathbb{P}$ is strategically $(<\lambda)$-complete by Observation 2.23, Define a binary relation $R^{\mathrm{pr}}$ by:

$\bar{T} R^{\text {pr }} T$ if and only if

- $\bar{T}=\left\langle T_{\alpha}: \alpha<\delta\right\rangle$ is a $\leq \mathbb{P}^{-}$-increasing sequence of conditions from $\mathbb{P}$ such that $\delta<\lambda$ is a limit ordinal and for $\alpha<\delta$ we have $\alpha \leq \ln \left(\operatorname{root}\left(T_{\alpha}\right)\right)<\delta$, and

- $T \in \mathbb{P}$ is a $\leq_{\mathbb{P}}$-upper bound of the sequence $\bar{T}$. (Note that the previous bullet implies $\bigcap_{\alpha<\delta} T_{\alpha} \in \mathbb{P}$ is the least upper bound of $\bar{T}$.)

Clearly $R^{\text {pr }}$ is a $\lambda$-sequential ${ }^{+}$purity on $\mathbb{P}$. Let $N, h, \bar{f}, \overline{\mathcal{I}}, \gamma$ be as in the assumptions of Condition [3.6 $(1)(\odot)$ and $\bar{q}=\left\langle q_{\delta}: \delta \in \mathcal{S}\right.$ is limit $\rangle$ be a candidate. Suppose $T \in \mathbb{P} \cap N$. To construct a condition $T^{*} \geq T$ generic for $\bar{q}$ we choose by induction on $\alpha<\lambda$ conditions $T_{\alpha} \in \mathbb{P} \cap N$ as follows.

$(\nabla)_{1} T_{0} \geq T$ satisfies

$$
\left(\forall t \in T_{0}\right)\left(\left|\operatorname{succ}_{T_{0}}(t)\right|>1 \Rightarrow \operatorname{lh}(t) \notin \mathcal{S}\right),
$$

and if $\delta$ is a limit ordinal, then $T_{\delta}=\bigcap_{\xi<\delta} T_{\xi}$.

$(\nabla)_{2}$ Suppose that $\alpha=\delta+1, \delta \in \mathcal{S}[\gamma]$ is a limit ordinal, and $h \circ f_{\delta}$ is a $\leq_{\mathbb{P}^{-}}$ increasing sequence of conditions from $\mathbb{P} \cap N$ such that for all successor $\beta<\delta$ we have

$$
\beta \leq \operatorname{lh}\left(\operatorname{root}\left(h\left(f_{\delta}(\beta)\right)\right)\right)<\delta \text { and } T_{\beta} \leq h\left(f_{\delta}(\beta)\right) .
$$

Let $t_{\delta}=\bigcup_{\beta<\delta} \operatorname{root}\left(h\left(f_{\delta}(\beta)\right)\right)$ and $T_{\alpha}^{*}=\left\{t \in T_{\delta}: t_{\delta} \unlhd t \Rightarrow t \in q_{\delta}\right\}$. Pick $T_{\alpha} \in \mathbb{P}$ so that $T_{\alpha} \subseteq T_{\alpha}^{*}$ and $T_{\alpha} \cap{ }^{\alpha} \lambda=T_{\alpha}^{*} \cap{ }^{\alpha} \lambda$.

$(\nabla)_{3}$ If $\alpha=\delta+1$ and the clause $(\nabla)_{2}$ does not apply, then $T_{\alpha}=T_{\delta}$.

Suppose that $\alpha=\delta+1$ satisfies the assumptions of $(\nabla)_{2}$. Then $\bigcap_{\beta<\delta} h\left(f_{\delta}(\beta)\right) \in \mathbb{P}$ and $T_{\delta} \leq \bigcap_{\beta<\delta} h\left(f_{\delta}(\beta)\right) \leq q_{\delta}$ and $t_{\delta} \unlhd \operatorname{root}\left(\bigcap_{\beta<\delta} h\left(f_{\delta}(\beta)\right)\right) \unlhd \operatorname{root}\left(q_{\delta}\right)$. Also $\operatorname{lh}\left(t_{\delta}\right)=$ $\delta \in \mathcal{S}$ so, by demand $(\nabla)_{1},\left|\operatorname{succ}_{T_{\delta}}\left(t_{\delta}\right)\right|=1$. Hence we may conclude that the sequence $\left\langle T_{\alpha}: \alpha<\lambda\right\rangle$ satisfies the assumptions of Lemma 2.24 At limit stages $\delta<\lambda$ this implies that $T_{\delta}=\bigcap_{\beta<\delta} T_{\beta} \in \mathbb{P}$ (so the construction can be carried out) and for $\lambda$ we get $T^{*} \stackrel{\text { def }}{=} \bigcap_{\alpha<\lambda} T_{\alpha} \in \mathbb{P}$.

Claim 3.9.2. $T^{*}$ is $(N, \mathbb{P})$-generic in the standard sense.

Proof of the Claim. Assume that $\xi_{0}<\lambda$ and $T^{+} \geq_{\mathbb{P}} T^{*}$. Build inductively a sequence $\left\langle S_{\alpha}: \alpha<\lambda\right\rangle$ of conditions in $\mathbb{P}$ such that

- $T^{+} \leq S_{\beta} \leq S_{\alpha}, \operatorname{lh}\left(\operatorname{root}\left(S_{\beta}\right)\right)<\operatorname{lh}\left(\operatorname{root}\left(S_{\alpha}\right)\right)$ for $\beta<\alpha<\lambda$, and 
- $S_{\alpha+1} \in \bigcap_{\xi \leq \alpha} \mathcal{I}_{\xi}$, and if $\alpha$ is limit then $S_{\alpha}=\bigcap_{\beta<\alpha} S_{\beta}$ (for all $\alpha<\lambda$ ).

Let $s_{\alpha}=\operatorname{root}\left(S_{\alpha}\right)$. Since $T_{\alpha} \supseteq T^{+} \supseteq S_{\alpha}$ we see that $s_{\alpha} \in T_{\alpha}$ and $\left(T_{\alpha}\right)_{s_{\alpha}} \leq S_{\alpha}$. For some limit ordinal $\delta \in \mathcal{S}[\gamma]$ we have $\xi_{0}<\delta$ and $\operatorname{lh}\left(s_{\alpha}\right)<\delta$ for $\alpha<\delta$ and $h \circ f_{\delta}=\left\langle\left(T_{\alpha}\right)_{s_{\alpha}}: \alpha<\delta\right\rangle$. Then $h \circ f_{\delta} R^{\mathrm{pr}} S_{\delta}$ and $S_{\delta} \in \bigcap \mathcal{I}_{\alpha}$. Hence also $q_{\delta} \in \bigcap_{\alpha<\delta} \mathcal{I}_{\alpha}$ (in particular $\left.q_{\delta} \in \mathcal{I}_{\xi_{0}} \cap N\right)$. Since for $t_{\delta}=\bigcup_{\alpha<\delta}^{\alpha<\delta} s_{\alpha}$ we have $q_{\delta} \leq$ $\left(T_{\delta+1}\right)_{t_{\delta}} \leq\left(T^{*}\right)_{t_{\delta}} \leq\left(T^{+}\right)_{t_{\delta}}$ we may conclude $\mathcal{I}_{\xi_{0}} \cap N$ is predense above $T^{*}$.

Let us show that $T^{*}$ is generic for $\bar{q}$ over $N, h, \mathbb{P}, R^{\mathrm{pr}}, \bar{f}, \mathcal{S}, \gamma$. Consider the following strategy st for Generic in the game $\partial_{\gamma}^{\mathcal{S}}\left(T^{*}, N, h, \mathbb{P}, R^{\mathrm{pr}}, \bar{f}, \bar{q}\right)$. At a stage $i \in \mathcal{S}[\gamma]$, when a sequence $\left\langle r_{j}^{-}, r_{j}, C_{j}: j<i\right\rangle$ has been already constructed, Generic picks $r_{i}^{-}, r_{i}, C_{i}$ so that

$(\nabla)_{4}$ if $i$ is limit, then $r_{i}^{-}=\bigcap_{j<i} r_{j}^{-}, r_{i}=\bigcap_{j<i} r_{j}$ and $C_{i}=\bigcap_{j<i} C_{j}$,

$(\nabla)_{5}$ if $i$ is a successor, say $i=j+1$, then $r_{i}^{-}, r_{i}$ are chosen so that $r_{j} \leq$ $r_{i}, \operatorname{root}\left(r_{j}\right) \triangleleft \operatorname{root}\left(r_{i}^{-}\right), r_{j}^{-} \leq r_{i}^{-}, T_{i} \leq r_{i}^{-}$and $r_{i}^{-} \leq r_{i}$. Also, $C_{i}=$ $\left[\operatorname{lh}\left(\operatorname{root}\left(r_{i}\right)\right)+1001, \lambda\right)$.

As in the previous part one uses Claim 3.9 .2 to show that the choices are indeed possible and that there are always legal moves for Antigeneric.

Suppose now that $\left\langle r_{i}^{-}, r_{i}, C_{i}: i<\lambda\right\rangle$ is a play of $\partial_{\gamma}^{\mathcal{S}}\left(T^{*}, N, h, \mathbb{P}, R^{\mathrm{pr}}, \bar{f}, \bar{q}\right)$ in which Generic follows the strategy st described above. Let $\delta \in \mathcal{S}[\gamma] \cap \bigcap_{i<\delta} C_{i}$ be a limit ordinal such that $h \circ f_{\delta}$ is an increasing sequence of conditions in $\mathbb{P}$ satisfying

$$
h\left(f_{\delta}(i)\right)=r_{i}^{-} \quad \text { for all successor } i<\delta \text {. }
$$

Then, by $(\nabla)_{5}+(\nabla)_{4}$, we have that $i \leq \operatorname{lh}\left(\operatorname{root}\left(h\left(f_{\delta}(i)\right)\right)\right)<\delta$ and $h\left(f_{\delta}(i)\right) \geq T_{i}$ (for all successor $i<\delta$ ). Therefore, letting $t_{\delta}=\bigcup_{i<\delta} \operatorname{root}\left(r_{i}^{-}\right)$and using $(\nabla)_{2}$ we conclude $q_{\delta} \leq\left(T_{\delta+1}\right)_{t_{\delta}} \leq\left(T^{*}\right)_{t_{\delta}} \leq\left(r_{\delta}\right)_{t_{\delta}}=r_{\delta}$ and $h \circ f_{\delta} R^{\mathrm{pr}} r_{\delta}$.

(h) In both cases the arguments are almost identical, so we will consider $\mathbb{P}=\mathbb{H}_{\lambda}$ only. Define a binary relation $R^{\mathrm{pr}}$ by:

$\bar{p} R^{\mathrm{pr}} p \quad$ if and only if

- $\bar{p}=\left\langle\left(s_{\alpha}, g_{\alpha}\right): \alpha<\delta\right\rangle$ is a $\leq_{\mathbb{H}_{\lambda}}$-increasing sequence of conditions from $\mathbb{H}_{\lambda}$ of limit length $\delta<\lambda$, and

- $p=(s, g) \in \mathbb{H}_{\lambda}$ is an upper bound to $\bar{p}$ with $s=\bigcup_{\alpha<\delta} s_{\alpha}$.

Clearly, $R^{\text {pr }}$ is a $\lambda^{+}$-sequential purity on $\mathbb{H}_{\lambda}$. Suppose $N, h, \bar{f}, \overline{\mathcal{I}}, \gamma$ are as in $3.6(\odot)$ and let $\bar{q}$ be a candidate for these parameters. We claim that every condition from $\mathbb{H}_{\lambda} \cap N$ is generic for $\bar{q}$. So let $(s, g) \in \mathbb{H}_{\lambda} \cap N$. Consider the following strategy st for Generic in $\partial_{\gamma}^{\mathcal{S}}\left((s, g), N, h, \mathbb{H}_{\lambda}, R^{\mathrm{pr}}, \bar{f}, \bar{q}\right)$. At a stage $i \in \mathcal{S}[\gamma]$ of the play, after a sequence $\left\langle\left(s_{j}^{-}, g_{j}^{-}\right),\left(s_{j}, g_{j}\right), C_{j}: j<i\right\rangle$ was already constructed, Generic is instructed to choose $\left(s_{i}^{-}, g_{i}^{-}\right),\left(s_{i}, g_{i}\right)$ and $C_{i}$ as follows.

$(\bullet)_{1}$ If $i=i_{0}+1$ is a successor, then $g_{i}^{-}=g_{i_{0}}^{-}, g_{i}=g_{i_{0}}, s_{i}^{-}=s_{i}=s_{i_{0}} \prec\left\langle g_{i_{0}}\left(\operatorname{lh}\left(s_{i_{0}}\right)\right)\right\rangle$ and $C_{i}=\left[\operatorname{lh}\left(s_{i_{0}}\right)+1001, \lambda\right)$.

$(\bullet)_{2}$ If $i \in \mathcal{S}[\gamma]$ is limit and $h(f(j))=\left(s_{j}^{-}, g_{j}^{-}\right)$for each successor $j<i$, and $q_{i}=\left(s^{*}, g^{*}\right)$ (note that necessarily $\left.s^{*}=\bigcup_{j<i} s_{j}^{-}\right)$, then $s_{i}^{-}=s_{i}=s^{*}, C_{i}=\lambda$ 
and for $\alpha<\lambda$ :

$g_{i}(\alpha)=\sup \left\{g_{j}(\alpha), g^{*}(\alpha): j<i\right\}$ and $g_{i}^{-}(\alpha)=\sup \left\{g_{j}^{-}(\alpha), g^{*}(\alpha): j<i\right\}$.

$(\bullet)_{3}$ If $i \in \mathcal{S}[\gamma]$ is limit but we are not in the previous case, then $s_{i}^{-}=s_{i}=\bigcup_{j<i} s_{j}^{-}$, $g_{i}(\alpha)=\sup \left\{g_{j}(\alpha): j<i\right\}, g_{i}^{-}(\alpha)=\sup \left\{g_{j}^{-}(\alpha): j<i\right\}$ for each $\alpha<\lambda$.

Now check.

(i) Define a binary relation $R^{\mathrm{pr}}$ by:

$\bar{p} R^{\mathrm{pr}} p \quad$ if and only if $\quad \bar{p}=\left\langle p_{\alpha}: \alpha<\delta\right\rangle$ is a $\leq_{\mathbb{P}}$-increasing sequence of conditions from $\mathbb{P}$ which has an upper bound in $\mathbb{P}, \delta<\lambda$ is a limit ordinal and $p \in \mathbb{P}$ is an upper bound to $\bar{p}$.

Plainly, $R^{\text {pr }}$ is a $\lambda$-sequential ${ }^{+}$purity on $\mathbb{P}$. (Remember $\mathbb{P}$ is $(<\lambda)$-complete.) Assume $N, h, \bar{f}, \overline{\mathcal{I}}, \gamma, \bar{q}$ are as in $\left[3.6(1)(\odot)\right.$ and let $p \in N$. Let st $^{*} \in N$ be a winning strategy of Complete in the game $\partial_{0}^{\lambda+1}(\mathbb{P})$. By induction on $\alpha \leq \lambda$ choose conditions $p_{\alpha}^{*}, q_{\alpha}^{*} \in \mathbb{P}$ so that

$(\square)_{1}\left\langle p_{\alpha}^{*}, q_{\alpha}^{*}: \alpha \leq \lambda\right\rangle$ is a legal play of $\partial_{0}^{\lambda+1}(\mathbb{P})$ in which Complete follows her winning strategy $\mathbf{s t}^{*}$,

$(\square)_{2} p_{0}^{*}=p, p_{\alpha}^{*}, q_{\alpha}^{*} \in N$ for all $\alpha<\lambda$,

$(\square)_{3} p_{\alpha+1}^{*} \in \bigcap_{\beta<\alpha} \mathcal{I}_{\beta}$ for each $\alpha<\lambda$,

$(\square)_{4}$ if $\delta \in \mathcal{S}$ is limit and $q_{\delta} \geq p_{\alpha}^{*}$ for all $\alpha<\delta$, then $p_{\delta}^{*}=q_{\delta}$.

(Clearly the construction is possible.) We will argue that the condition $p_{\lambda}^{*}$ is generic for the candidate $\bar{q}$ over $N, h, \mathbb{P}, R^{\mathrm{pr}}, \bar{f}, \mathcal{S}, \gamma$. Consider a strategy st ${ }^{+}$of Generic in $\partial_{\gamma}^{\mathcal{S}}\left(p_{\lambda}^{*}, N, h, \mathbb{P}, R^{\mathrm{pr}}, \bar{f}, \bar{q}\right)$ which instructs her to choose $r_{i}^{-}, r_{i}, C_{i}$ (for $i \in \mathcal{S}[\gamma]$ ) and side ordinals $\alpha_{i}<\lambda$ (for $i<\lambda$ ) so that the following demands $(\square)_{5}-(\square)_{9}$ are satisfied.

$(\square)_{5} r_{i}^{-} \in \mathbb{P} \cap N$ and $r_{i} \in \mathbb{P}$.

$(\square)_{6} r_{i}^{-} \leq r_{i}$ and if $j<i$ then $r_{j} \leq r_{i}$ and $\gamma<\alpha_{j}<\alpha_{i}$.

$(\square)_{7} \alpha_{i}<\lambda$ is the smallest ordinal above $\{\gamma+1\} \cup\left\{\alpha_{j}+1: j<i\right\}$ such that $r_{i}^{-} \leq p_{\alpha_{i}}^{*}$ and then $r_{i+1}^{-}=p_{\alpha_{i}}^{*}, r_{i+1}=r_{i}$ and $C_{i+1}=\left[\alpha_{i}+1001, \lambda\right)$.

$(\square)_{8}$ If $\delta \in \mathcal{S}[\gamma]$ is limit and $q_{\delta} \geq r_{i}^{-}$for all $i<\delta$, and the family $\left\{q_{\delta}\right\} \cup\left\{r_{i}: i<\delta\right\}$ has an upper bound in $\mathbb{P}$, then $r_{\delta}^{-}=q_{\delta}$ and $r_{\delta} \in \mathbb{P}$ is the $<_{\chi}^{*}$-first condition stronger than $q_{\delta}$ and stronger than all $r_{i}$ for $i<\delta$, and $C_{i}=\lambda$.

$(\square)_{9}$ If $\delta \in \mathcal{S}[\gamma]$ is limit but we are not in the case of $(\square)_{8}$, then $\left(r_{\delta}^{-}, r_{\delta}, C_{\delta}\right)$ is the $<_{\chi}^{*}$-first legal move of Generic after $\left\langle r_{i}^{-}, r_{i}, C_{i}: i<\delta\right\rangle$ which satisfies also $r_{\delta}^{-} \leq r_{\delta}$.

The above conditions fully define a strategy $\mathbf{s t}^{+}$for Generic. Concerning clause $(\square)_{7}$ and the choice of $\alpha_{i}$, note that by $(\square)_{3}$ there is an $\alpha<\lambda$ such that either the conditions $p_{\alpha}^{*}, r_{i}^{-}$are incompatible or $p_{\alpha}^{*} \geq r_{i}^{-}$. Since $r_{i} \geq p_{\lambda}^{*} \geq p_{\alpha}^{*}$ and $r_{i} \geq r_{i}^{-}$, the conditions $p_{\alpha}^{*}, r_{i}^{-}$are always compatible, and hence there is $\alpha_{i}<\lambda$ such that $p_{\alpha_{i}}^{*} \geq r_{i}^{-}$. Note that the condition $p_{\lambda}^{*}$ is $(N, \mathbb{P})$-generic in the standard sense (by $(\square)_{3}$ ) and also for each $j<\lambda$ there is an upper bound to $\left\{r_{i}: i<j\right\}$ (and this upper bound is $(N, \mathbb{P})$-generic). Hence, for both players, there are always legal moves during a play in which Generic followed $\mathbf{s t}^{+}$so far ( $\operatorname{satisfying} r_{i}^{-} \leq r_{i}$ ). So, in particular, clause $(\square)_{9}$ completes the description of $\mathbf{s t}^{+}$.

Let us argue that $\mathbf{s t}^{+}$is a winning strategy for Generic. To this end, assume $\left\langle r_{i}^{-}, r_{i}, C_{i}: i<\lambda\right\rangle$ is a play in which Generic follows $\mathbf{s t}^{+}$and let $\left\langle\alpha_{i}: i<\lambda\right\rangle$ be the 
side ordinals chosen by her. Suppose $\delta \in \mathcal{S}[\gamma] \cap \bigcap_{i<\delta} C_{i}$ is a limit ordinal such that $h \circ f_{\delta}$ is an increasing sequence of conditions from $\mathbb{P} \cap N$ satisfying

$$
h\left(f_{\delta}(i+1)\right)=r_{i+1}^{-} \quad \text { for all } i<\delta .
$$

Then also $i \leq \alpha_{i}<\delta$ for each $i<\delta$ so by $(\square)_{7}$ we have

$$
(\forall i<\delta)(\exists \alpha<\delta)\left(r_{i}^{-} \leq p_{\alpha}^{*}\right) \quad \text { and } \quad(\forall \alpha<\delta)(\exists i<\delta)\left(p_{\alpha}^{*} \leq r_{i}^{-}\right) .
$$

Thus the sequences $\left\langle p_{\alpha}^{*}: \alpha<\delta\right\rangle$ and $\left\langle r_{i}^{-}: i<\delta\right\rangle$ and also $h \circ f_{\delta}$ have the same upper bounds. Now, by 3.5 (2) (a,b), we have $q_{\delta} \geq h \circ f_{\delta}(i+1)=r_{i+1}^{-}$for all $i<\delta$, and hence $q_{\delta}$ is stronger than all $p_{\alpha}^{*}$ for $\alpha<\delta$. By $(\square)_{4}$ we get $q_{\delta}=p_{\delta}^{*} \leq p_{\lambda}^{*} \leq r_{i}$ (for $i<\delta$ ). Thus clause $(\square)_{8}$ applies and $r_{\delta}^{-}=q_{\delta} \leq r_{\delta}$ so also $h \circ f_{\delta} R^{\mathrm{pr}} r_{\delta}$.

\section{The iteration THEOREM}

Here we will show the main result: pure sequential ${ }^{+}$properness is almost preserved in $\lambda$-support iterations. The proof of the theorem is somewhat similar to that of [14, Theorem 3.7], but there are major differences in the involved concepts.

Theorem 4.1. Assume Context [3.1, Let $\overline{\mathbb{Q}}=\left\langle\mathbb{P}_{\alpha}, \mathbb{Q}_{\alpha}: \alpha\left\langle\zeta^{*}\right\rangle\right.$ be a $\lambda$-support iteration such that for each $\alpha<\zeta^{*}$

$$
\vdash_{\mathbb{P}_{\alpha}} \text { “ } \mathbb{Q}_{\alpha} \text { is purely sequentially }{ }^{+} \text {proper over }(D, \mathcal{S}) \text {-semi diamonds ". }
$$

Then

(1) $\mathbb{P}_{\zeta^{*}}=\lim (\overline{\mathbb{Q}})$ is purely sequentially proper over $(D, \mathcal{S})$-semi diamonds.

(2) If, additionally, for each $\alpha<\zeta^{*}$

$$
\Vdash_{\mathbb{P}_{\alpha}} \text { “ } \mathbb{Q}_{\alpha} \text { is }(<\lambda) \text {-complete " }
$$

then $\mathbb{P}_{\zeta^{*}}$ is purely sequentially $y^{+}$proper over $(D, \mathcal{S})$-semi diamonds.

Proof. By adding trivial iterands to the tail of $\overline{\mathbb{Q}}$ we may assume that $\zeta^{*} \geq \lambda^{+}$(this

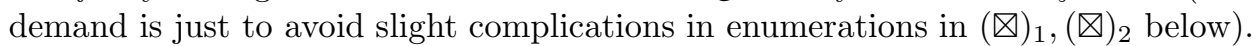

Assume that $\chi$ is a large enough regular cardinal and $N \prec\left(\mathcal{H}(\chi), \in,<_{\chi}^{*}\right),|N|=$ $\lambda,{ }^{<\lambda} N \subseteq N$ and $\lambda, \overline{\mathbb{Q}}, D, \mathcal{S}, \ldots \in N$. For $\xi<\zeta^{*}$ let $\mathbf{s t}_{\xi}^{0}$ be the $<_{\chi}^{*}$-first $\mathbb{P}_{\xi}$-name for a regular winning strategy of Complete in $\partial_{0}^{\lambda}\left(\mathbb{Q}_{\xi}\right)$ (so if $\xi \in \zeta^{*} \cap N$ then also $\left.\mathbf{s t}_{\xi}^{0} \in N\right)$. To define a $\lambda$-sequential purity $R^{\text {pr }}$ on $\tilde{\mathbb{P}}_{\zeta^{*}}$ we first fix

$(\bigotimes)_{1}$ a list $\left\langle\left(\tau_{i}, \zeta_{i}\right): i<\lambda\right\rangle$ of all pairs $(\underset{\sim}{\tau}, \zeta) \in N$ such that $\zeta \leq \zeta^{*}, \operatorname{cf}(\zeta) \geq \lambda$ and $\tau$ is a $\mathbb{P}_{\zeta}$-name for an ordinal, and

$(\otimes)_{2}$ an increasing sequence $\bar{w}=\left\langle w_{i}: i<\lambda\right\rangle$ of closed subsets of $\zeta^{*}+1$ such that

$(\otimes)_{2}^{a} w_{0}=\left\{0, \zeta^{*}\right\}, \bigcup_{i<\lambda} w_{i}=\left(\zeta^{*}+1\right) \cap N$ and for each $i<\lambda$ we have $\left|w_{i}\right| \leq 2+i$ and

$(\otimes)_{2}^{b}$ if $i$ is limit then $w_{i}$ is the closure of $\bigcup_{j<i} w_{j}$, and

$(\otimes)_{2}^{c}$ if $i$ is a successor, say $i=j+1$, then for some $\xi \in w_{i} \cap \zeta_{j}$ we have $\sup \left(w_{j} \cap \zeta_{j}\right)<\xi$.

We also define a function $\Upsilon:\left(\zeta^{*}+1\right) \cap N \longrightarrow \lambda$ by

$(\otimes)_{3} \Upsilon(\varepsilon)=\min \left(i<\lambda: \varepsilon \in w_{i}\right)$ for $\varepsilon \in\left(\zeta^{*}+1\right) \cap N$. 
Then for every $\zeta \in \zeta^{*} \cap N$ we fix a $\mathbb{P}_{\zeta}$-name $\underset{\sim}{R}$ pr for a binary relation such that

$\Vdash_{\mathbb{P}_{\zeta}} \quad$ “ ${\underset{\sim}{\zeta}}_{\zeta}^{\mathrm{pr}}$ is a $\lambda$-sequential ${ }^{+}$purity on $\mathbb{Q}_{\zeta}$ such that if $N\left[G_{\mathbb{P}_{\zeta}}\right]$ is a model of the right form, then $R_{\zeta}^{\mathrm{pr}}$ witnesses the pure sequential ${ }^{+}$properness of $\mathbb{Q}_{\zeta}$ for $N\left[G_{\mathbb{P}_{\zeta}}\right]$ ".

Now, for $\zeta \leq \zeta^{*}$, we define a binary relation $R^{\text {pr }}\lceil\zeta$ by

$(\otimes)_{4} \bar{p} R^{\mathrm{pr}}\lceil\zeta p$ if and only if

$(\bigotimes)_{4}^{a} \bar{p}=\left\langle p_{\alpha}: \alpha<\delta\right\rangle$ is an increasing sequence of conditions from $\mathbb{P}_{\zeta}$ of a limit length $\delta<\lambda, p \in \mathbb{P}_{\zeta}$ is an upper bound to $\bar{p}$ and

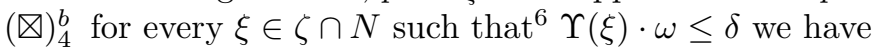

$$
p \nmid \xi \Vdash_{\mathbb{P}_{\xi}} \text { “ }\left\langle p_{\Upsilon(\xi)+\alpha}(\xi): \alpha<\delta\right\rangle \underset{\sim}{R_{\xi}^{\mathrm{pr}}} p(\xi) " .
$$

The relation $R^{\mathrm{pr}}$ is $R^{\mathrm{pr}}\left\lceil\zeta^{*}\right.$.

One may wonder why we use $\Upsilon \cdot \omega$ in $(\otimes)_{4}^{b}$. When playing the game $\partial_{1}^{\mathcal{S}}$, we will play its variant on each coordinate from $N \cap \zeta^{*}$. However, at a stage $\delta$ of the main game, we will look at coordinates from $w_{\delta}$ only, so we will start our coordinate games with some delay. At stage $j=\Upsilon(\xi)+i$, the length of the play constructed so far at coordinate $\xi$ is $i$. It may happen that $i \in \mathcal{S}$ but $\Upsilon(\xi)+i \notin \mathcal{S}$. Since we do want to control who is making a move at every coordinate, it makes sense to demand that $\delta \backslash \Upsilon(\xi)$ has order type $\delta$ whenever $\delta$ is of any potential importance. This translates to the demand that at coordinate $\xi \in N \cap \zeta^{*}$ we play game $\partial_{\Upsilon(\xi) \cdot \omega}^{\mathcal{S}}$ and relevant $\delta$ s are not smaller that $\Upsilon(\xi) \cdot \omega$.

Clearly, for each $\zeta \leq \zeta^{*}$,

- $R^{\text {pr }} \zeta \zeta$ is a $\lambda$-sequential purity on $\mathbb{P}_{\zeta}$,

- if each $\mathbb{Q}_{\alpha}$ is (forced to be) $(<\lambda)$-complete7, then $R^{\text {pr }}\left\lceil\zeta\right.$ is a $\lambda$-sequential ${ }^{+}$ purity,

- if $\left\langle p_{\alpha}: \alpha<\delta\right\rangle\left(R^{\operatorname{pr}}\lceil\zeta) p\right.$ and $\zeta^{\prime}<\zeta$ then $\left\langle p_{\alpha} \mid \zeta^{\prime}: \alpha<\delta\right\rangle\left(R^{\operatorname{pr}}\left\lceil\zeta^{\prime}\right) p\left\lceil\zeta^{\prime}\right.\right.$.

We will show that the relation $R^{\mathrm{pr}}$ witnesses the pure sequential properness of $\mathbb{P}_{\zeta^{*}}$ for $N$. So let $h, \overline{\mathcal{I}}, \bar{f}, \bar{q}$ and $p$ be as in $(\odot)$ of Definition $3.6(1)$, that is they satisfy the following conditions $(\otimes)_{5}-(\bigotimes)_{8}$.

$(\otimes)_{5}$ A function $h: \lambda \longrightarrow N$ is such that $\mathbb{P}_{\zeta^{*}} \cap N \subseteq \operatorname{rng}(h)$.

$(\otimes)_{6} \quad$ A sequence $\bar{f}=\left\langle f_{\delta}: \delta \in \mathcal{S}\right\rangle$ is a $(D, \mathcal{S}, h)$-semi diamond for $\mathbb{P}_{\zeta^{*}}$ over $N$.

$(\otimes)_{7} \overline{\mathcal{I}}=\left\langle\mathcal{I}_{\alpha}: \alpha<\lambda\right\rangle$ lists all open dense subsets of $\mathbb{P}_{\zeta^{*}}$ from $N$.

$(\otimes)_{8} \bar{q}=\left\langle q_{\delta}: \delta \in \mathcal{S}\right.$ is limit $\rangle$ is an $\left(N, h, \mathbb{P}_{\zeta^{*}}, R^{\mathrm{pr}}, \bar{f}, \overline{\mathcal{I}}\right)$-candidate, $p \in \mathbb{P}_{\zeta^{*}} \cap N$.

We also have an ordinal $\gamma<\lambda$, but replacing $\mathcal{S}$ with $\mathcal{S}[\gamma]$ in the following arguments makes not much difference, so we may pretend that $\gamma=1$ and $\mathcal{S}[\gamma]=\mathcal{S}$.

We will define a condition $r \geq p$ such that Generic will have a winning strategy in the game $\partial_{1}^{\mathcal{S}}\left(r, N, h, \mathbb{P}_{\zeta^{*}}, R^{\text {pr }}, \bar{f}, \bar{q}\right)$, that is $r$ will be generic for the candidate $\bar{q}$. This condition will be defined essentially by considering the game $\partial_{\Upsilon(\zeta) \cdot \omega}^{\mathcal{S}}$ on each coordinate $\zeta \in \zeta^{*} \cap N$. The proof that it has the required properties will be by induction on $\zeta$. Therefore, we have to introduce both the restrictions of our parameters to $\zeta$ (denoted with superscript $[\zeta]$ ) and the parameters at coordinate $\zeta$ (denoted with superscript $\langle\zeta\rangle$ ). We will also need other auxiliary notions, definitions and claims. First note that if we modify $f_{\delta}$ (and $q_{\delta}$ ) for all limit $\delta \in \mathcal{S}$ such that $h \circ f_{\delta}$ is not an increasing sequence of conditions from $\mathbb{P}_{\zeta^{*}}$, then we can make the

\footnotetext{
6 the "." stands for the ordinal multiplication

${ }^{7}$ We need this assumption to get bounds on coordinates $\xi<\zeta$ for which $(\otimes)_{4}^{b}$ is not "active".
} 
winning for Generic only more difficult. So we may assume that for each limit $\delta \in \mathcal{S}$ we have

$(\bigotimes)_{9} h \circ f_{\delta}$ is a $\leq_{\mathbb{P}_{\zeta^{*}}}$-increasing sequence of members of $\mathbb{P}_{\zeta^{*}} \cap N$.

For $\zeta \in N \cap\left(\zeta^{*}+1\right)$ we define

$(\otimes)_{10} h^{[\zeta]}: \lambda \longrightarrow N$ is a function such that for each $\alpha<\lambda$ :

$h^{[\zeta]}(\alpha)=h(\alpha)\left\lceil\zeta\right.$ provided $h(\alpha)$ is a function, and $h^{[\zeta]}(\alpha)=\emptyset$ otherwise,

$(\otimes)_{11} \overline{\mathcal{I}}^{[\zeta]}=\left\langle\mathcal{I}_{\alpha}^{[\zeta]}: \alpha<\lambda\right\rangle$, where $\mathcal{I}_{\alpha}^{[\zeta]}=\left\{p\left\lceil\zeta: p \in \mathcal{I}_{\alpha}\right\}\right.$.

Clearly $\left\langle q_{\delta} \mid \zeta: \zeta \in \mathcal{S}\right\rangle$ does not have to be a $\left(N, h^{[\zeta]}, \mathbb{P}_{\zeta}, R^{\operatorname{pr}}\left\lceil\zeta, \bar{f}, \overline{\mathcal{I}}^{[\zeta]}\right)\right.$-candidate. To define suitable $q^{[\zeta]}$ we need the concept of saturated conditions. Suppose that $\delta \in \mathcal{S}$ is a limit ordinal, $\zeta \in w_{\delta}$ and $q^{*} \in \mathbb{P}_{\zeta}$. We say that the condition $q^{*}$ is saturated over $w_{\delta}, h, \overline{\mathcal{I}}$ at $\zeta$ if one of the following two possibilities $(\mathbb{\nabla})_{12}^{a}$ or $(\otimes)_{12}^{b}$ holds:

$(\otimes)_{12}^{a} \quad$ (i) $\left(h^{[\zeta]} \circ f_{\delta}\right)\left(R^{\mathrm{pr}}\lceil\zeta) q^{*}\right.$ and $q^{*} \in \bigcap_{\alpha<\delta} \mathcal{I}_{\alpha}^{[\zeta]}$, but

(ii) if $\zeta^{\prime} \in w_{\delta} \backslash(\zeta+1)$ then there is no $q^{\prime} \in \mathbb{P}_{\zeta^{\prime}}$ such that $q^{*} \leq q^{\prime}$ and

$$
\left(h^{\left[\zeta^{\prime}\right]} \circ f_{\delta}\right)\left(R^{\mathrm{pr}}\left\lceil\zeta^{\prime}\right) q^{\prime} \quad \text { and } \quad q^{\prime} \in \bigcap_{\alpha<\delta} \mathcal{I}_{\alpha}^{\left[\zeta^{\prime}\right]} .\right.
$$

$(\otimes)_{12}^{b} \quad$ (i) $0<\zeta=\sup \left(w_{\delta} \cap \zeta\right)$ and for each $\xi \in w_{\delta} \cap \zeta$ we have

$$
\left(h^{[\xi]} \circ f_{\delta}\right)\left(R^{\mathrm{pr}} \mid \xi\right) q^{*} \mid \xi \quad \text { and } \quad q^{*} \mid \xi \in \bigcap_{\alpha<\delta} \mathcal{I}_{\alpha}^{[\xi]}, \quad \text { but }
$$

(ii) there is no condition $q^{\prime} \in \mathbb{P}_{\zeta}$ such that $q^{*} \leq q^{\prime}$ and

$$
\left(h^{[\zeta]} \circ f_{\delta}\right)\left(R^{\mathrm{pr}}\lceil\zeta) q^{\prime} \quad \text { and } \quad q^{\prime} \in \bigcap_{\alpha<\delta} \mathcal{I}_{\alpha}^{[\zeta]} .\right.
$$

Claim 4.1.1. Suppose $\delta \in \mathcal{S}$ is a limit ordinal. Then there exist $\zeta \in w_{\delta}$ and $q^{*} \in \mathbb{P}_{\zeta} \cap N$ such that $q^{*}$ is saturated over $w_{\delta}, h, \overline{\mathcal{I}}$ at $\zeta$.

Proof of the Claim. Let $\left\langle\alpha_{\xi}: \xi \leq \xi^{*}\right\rangle$ be the increasing enumeration of $w_{\delta}$ (so it is a continuous sequence and $\alpha_{\xi^{*}}=\zeta^{*}$ and $\xi^{*}<|\delta|^{+}$). We construct the condition $q^{*}$ as an upper bound to an increasing sequence $\left\langle q_{\xi}^{+}: \xi<\xi_{0}\right\rangle, \xi_{0} \leq \xi^{*}$. Conditions $q_{\xi}^{+}$are chosen so that $q_{\xi}^{+} \in \mathbb{P}_{\alpha_{\xi}} \cap N$ and they satisfy $(\otimes)_{12}^{a}$ (i). To make sure that we can get pass the limit steps, at coordinates $\varepsilon \in N \cap \zeta^{*}$ which are not relevant for $R^{\mathrm{pr}}$ (i.e., with $\Upsilon(\varepsilon) \cdot \omega>\delta$ ) we employ strategic completeness. (This forces us to introduce auxiliary conditions $q_{\xi}^{*}$.) We do not have to worry about coordinates relevant for $R^{\mathrm{pr}}$ (i.e., with $\Upsilon(\varepsilon) \cdot \omega \leq \delta$ ) as there the appropriate bounds exist by 3.4(c). The construction may stop at some stage $\xi_{0}<\xi^{*}$ because we arrived to a saturated condition. If the procedure does not stop before $\xi^{*}$, then we get a condition saturated at $\zeta^{*}$.

Let us present this argument with all technical details. So we attempt to choose inductively a sequence $\left\langle q_{\xi}^{+}, q_{\xi}^{*}: \xi \leq \xi^{*}\right\rangle$ so that $q_{\xi}^{+}, q_{\xi}^{*} \in \mathbb{P}_{\alpha_{\xi}} \cap N$ and

(i) if $\xi^{\prime}<\xi^{\prime \prime} \leq \xi^{*}$ then $q_{\xi^{\prime}}^{+} \leq q_{\xi^{\prime}}^{*} \leq q_{\xi^{\prime \prime}}^{+} \leq q_{\xi^{\prime \prime}}^{*}$,

(ii) if $\varepsilon \in N \cap \zeta^{*}$ and $\Upsilon(\varepsilon) \cdot \omega>\delta$, then for every $\xi \leq \xi^{*}$,

$$
q_{\xi}^{*}\left\lceil\varepsilon \Vdash_{\mathbb{P}_{\varepsilon}} \quad \text { “ }\left\langle q_{\xi^{\prime}}^{+}(\varepsilon), q_{\xi^{\prime}}^{*}(\varepsilon): \xi^{\prime} \leq \xi\right\rangle \text { is a legal partial play of } \partial_{0}^{\lambda}\left(\mathbb{Q}_{\varepsilon}\right)\right.
$$

in which Complete uses her regular winning strategy $\mathbf{s t}_{\varepsilon}^{0} "$, 
(iii) if $\varepsilon \in N \cap \zeta^{*}$ and $\Upsilon(\varepsilon) \cdot \omega \leq \delta$, then $q_{\xi}^{+}(\varepsilon)=q_{\xi}^{*}(\varepsilon)$,

(iv) $\left(h^{\left[\alpha_{\xi}\right]} \circ f_{\delta}\right)\left(R^{\mathrm{pr}}\left\lceil\alpha_{\xi}\right) q_{\xi}^{+}\right.$and $q_{\xi}^{+} \in \bigcap_{\alpha<\delta} \mathcal{I}_{\alpha}^{\left[\alpha_{\xi}\right]}$.

If we have arrived to a limit stage $\xi_{0}$ of the construction and we have defined successfully $\left\langle q_{\xi}^{+}, q_{\xi}^{*}: \xi<\xi_{0}\right\rangle$ (so that conditions (i)-(iv) are satisfied for all $\xi<\xi_{0}$ ), then, remembering ${ }^{<\lambda} N \subseteq N$ and $\delta<\lambda$, we may find a condition $q_{\xi_{0}}^{\diamond} \in \mathbb{P}_{\alpha_{\xi_{0}}} \cap N$ which is above all $q_{\xi}^{+}$(for $\left.\xi<\xi_{0}\right)$ and satisfies $8\left(h^{\left[\alpha_{\xi_{0}}\right]} \circ f_{\delta}\right)\left(R^{\mathrm{pr}}\left\lceil\alpha_{\xi_{0}}\right) q_{\xi_{0}}^{\diamond}\right.$. Then also $q_{\xi_{0}}^{\diamond}\left\lceil\alpha_{\xi} \in \bigcap_{\alpha<\delta} \mathcal{I}_{\alpha}^{\left[\alpha_{\xi}\right]}\right.$ for all $\xi<\xi_{0}$, so either $q_{\xi_{0}}^{\diamond}$ is saturated by $(\nabla)_{12}^{b}$ (and then we stop), or else we may pick $q_{\xi_{0}}^{+} \in \mathbb{P}_{\alpha_{\xi_{0}}} \cap N$ stronger than $q_{\xi_{0}}^{\diamond}$ and satisfying the demand in (iv), and then we pick $q_{\xi_{0}}^{*} \in \mathbb{P}_{\alpha_{\xi_{0}}} \cap N$ by (ii)+(iii).

Now, if we arrived to a successor stage $\xi=\xi_{0}+1 \leq \xi^{*}$ and we have defined $q_{\xi_{0}}^{+}, q_{\xi_{0}}^{*}$, then either $q_{\xi_{0}}^{*}$ is saturated by $(\otimes)_{12}^{a}$ (and then we stop), or else we may pick $q_{\xi_{0}+1}^{+} \in \mathbb{P}_{\alpha_{\xi_{0}+1}} \cap N$ stronger than $q_{\xi_{0}}^{*}$ and such that (iv) holds, and then we choose $q_{\xi_{0}+1}^{*}$ by (ii) + (iii). (We also stipulate $q_{0}^{+}=q_{0}^{*}=\emptyset_{\mathbb{P}_{0}} \in \mathbb{P}_{0}$.)

If we did manage to carry out the construction up to $\xi^{*}$ and we defined successfully $q_{\xi^{*}}^{+}$, then it is vacuously saturated by $(\otimes)_{12}^{a}$.

Now, for each limit $\delta \in \mathcal{S}$ we fix a pair $\left(q_{\delta}^{*}, \zeta_{\delta}^{*}\right) \in N$ so that

$(\bigotimes)_{13}^{a} \quad q_{\delta}^{*}$ is saturated over $w_{\delta}, h, \overline{\mathcal{I}}$ at $\zeta_{\delta}^{*}$, and

$(\otimes)_{13}^{b}$ if the condition $q_{\delta}$ given by $(\otimes)_{8}$ is saturated over $w_{\delta}, h, \overline{\mathcal{I}}$ at $\zeta^{*}$, then $q_{\delta}^{*}=q_{\delta}, \zeta_{\delta}^{*}=\zeta^{*}$.

Next for $\zeta \in\left(\zeta^{*}+1\right) \cap N$ we define

$(\otimes)_{14} \bar{q}^{[\zeta]}=\left\langle q_{\delta}^{[\zeta]}: \delta \in \mathcal{S}\right.$ is limit $\rangle$, where for a limit $\delta \in \mathcal{S}$ we set

- if $\zeta<\zeta_{\delta}^{*}$ then $q_{\delta}^{[\zeta]}=q_{\delta}^{*}\lceil\zeta$,

- if $\zeta=\zeta_{\delta}^{*}, q_{\delta}^{*} \in \bigcap_{\alpha<\delta} \mathcal{I}_{\alpha}^{[\zeta]}$ and $\left(h^{[\zeta]} \circ f_{\delta}\right)\left(R^{\operatorname{pr}}\lceil\zeta) q_{\delta}^{*}\right.$, then $q_{\delta}^{[\zeta]}=q_{\delta}^{*}$,

- otherwise, $q_{\delta}^{[\zeta]}$ is the $<_{\chi}^{*}$-first condition in $\mathbb{P}_{\zeta}$ satisfying the demands in (a)-(c) of $3.5(2)$ with $h^{[\zeta]}, R^{\mathrm{pr}}\left\lceil\zeta, \overline{\mathcal{I}}^{[\zeta]}\right.$ here in place of $h, R^{\mathrm{pr}}, \overline{\mathcal{I}}$ there.

Claim 4.1.2. Let $\zeta \in N \cap\left(\zeta^{*}+1\right)$. Then

(1) $h^{[\zeta]}: \lambda \longrightarrow N$ is such that $\mathbb{P}_{\zeta} \cap N \subseteq \operatorname{rng}\left(h^{[\zeta]}\right)$,

(2) $\bar{f}$ is a $\left(D, \mathcal{S}, h^{[\zeta]}\right)$-semi diamond sequence for $\mathbb{P}_{\zeta}$,

(3) $\overline{\mathcal{I}}[\zeta]$ lists all open dense subsets of $\mathbb{P}_{\zeta}$ belonging to $N$,

(4) $\bar{q}^{[\zeta]}$ is an $\left(N, h^{[\zeta]}, \mathbb{P}_{\zeta}, R^{\text {pr }}\left\lceil\zeta, \bar{f}, \overline{\mathcal{I}}^{[\zeta]}\right)\right.$-candidate.

Proof of the Claim. Straightforward from the definitions.

For $\zeta \in \zeta^{*} \cap N$ we set

$(\otimes)_{15} \bar{f}^{\langle\zeta\rangle}=\left\langle f_{\delta}^{\langle\zeta\rangle}: \delta \in \mathcal{S}\right\rangle$, where for $\delta \in \mathcal{S}$ and $\alpha<\delta$ we let

$$
f_{\delta}^{\langle\zeta\rangle}(\alpha)= \begin{cases}f_{\delta}(\alpha) & \text { if } \delta<\Upsilon(\zeta) \cdot \omega, \\ f_{\delta}(\Upsilon(\zeta)+\alpha) & \text { if } \delta \geq \Upsilon(\zeta) \cdot \omega,\end{cases}
$$

and then we define $\mathbb{P}_{\zeta}$-names ${\underset{\sim}{h}}^{\langle\zeta\rangle}, \overline{\mathcal{I}}^{\langle\zeta\rangle}, \bar{\sim}^{\langle\zeta\rangle}$ so that

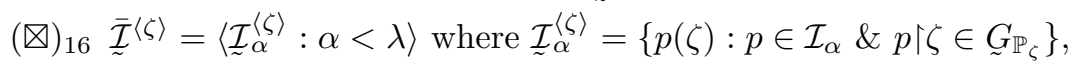

\footnotetext{
${ }^{8}$ Here we use the assumption that $\underset{\sim}{R}{ }_{\varepsilon}^{\mathrm{pr}}$ are sequential ${ }^{+}$purities rather than just sequential purities.
} 
$(\otimes)_{17} \underset{\sim}{h}\langle\zeta\rangle$ is a name for a function with domain $\lambda$ and such that for each $\gamma<\lambda$, if $h(\gamma)$ is a function, $\zeta \in \operatorname{dom}(h(\gamma))$ and $(h(\gamma))(\zeta)$ is a $\mathbb{P}_{\zeta}$-name, then $\stackrel{\sim}{h}^{\langle\zeta\rangle}(\gamma)=(h(\gamma))(\zeta)$, otherwise it is $\emptyset_{\mathbb{Q}_{\zeta}}$,

$(\otimes)_{18} \bar{q}^{\langle\zeta\rangle}=\left\langle q_{\delta}^{\langle\zeta\rangle}: \delta \in \mathcal{S}\right.$ is limit $\rangle$, where for a limit $\delta \in \mathcal{S}, q_{\delta}^{\langle\zeta\rangle}$ is the $<_{\chi}^{*}$-first $\tilde{\mathbb{P}}_{\zeta}$-name for a condition in $\mathbb{Q}_{\zeta}$ such that:

if $\zeta<\zeta_{\delta}^{*}$ and the condition $q_{\delta}^{*}(\zeta)=q_{\delta}^{[\zeta+1]}(\zeta)$ satisfies the demands in (a)(c) of Definition 3.5 (2) with $\underset{\sim}{h}\langle\zeta\rangle, f_{\delta}^{\langle\zeta\rangle},{\underset{\sim}{\zeta}}_{\zeta}^{\mathrm{pr}}, \tilde{\mathcal{I}}^{\langle\zeta\rangle}$ here in place of $h, f_{\delta}, R^{\mathrm{pr}}, \overline{\mathcal{I}}$ there, then $q_{\delta}^{\langle\zeta\rangle}=q_{\delta}^{*}(\zeta)$, otherwise, $q_{\delta}^{\langle\zeta\rangle}$ is any condition in $\mathbb{Q}_{\zeta}$ satisfying those demands.

Note that

$(\otimes)_{19}$ if $\delta \in \mathcal{S}$ is limit and $\zeta \in \zeta_{\delta}^{*} \cap N$ and $\Upsilon(\zeta) \cdot \omega \leq \delta$, then $q_{\delta}^{*} \mid \zeta \Vdash_{\mathbb{P}_{\zeta}}$ " $q_{\delta}^{\langle\zeta\rangle}=q_{\delta}^{*}(\zeta)$ ".

Claim 4.1.3. Assume that $\zeta \in N \cap \zeta^{*}$ and $r \in \mathbb{P}_{\zeta}$ is $\left(N, \mathbb{P}_{\zeta}\right)$-generic in the standard sense. Then the condition $r$ forces (in $\mathbb{P}_{\zeta}$ ) that:

(1) $N\left[G_{\mathbb{P}_{\zeta}}\right]$ is a model of the right form, $\overline{\mathcal{I}}^{\langle\zeta\rangle}$ lists all open dense subsets of $\mathbb{Q}_{\sim} \zeta$ which belong to $N\left[G_{\mathbb{P}_{\zeta}}\right]$ and the function $\underset{\sim}{h}\langle\zeta\rangle$ is such that $N\left[G_{\mathbb{P}_{\zeta}}\right] \cap \mathbb{Q}_{\sim} \subseteq$ $\operatorname{rng}\left(h^{\langle\zeta\rangle}\right)$, and

(2) $\bar{f}\langle\zeta\rangle$ is a $\left(D, \mathcal{S}, h_{\sim}^{\langle\zeta\rangle}\right)$-semi diamond for $\mathbb{Q}_{\sim}$ over $N\left[G_{\mathbb{P}_{\zeta}}\right]$, and

(3) $\bar{\sim}^{\langle\zeta\rangle}$ is an $\left(N\left[G_{\mathbb{P}_{\zeta}}\right], h^{\langle\zeta\rangle}, \mathbb{Q}_{\sim}, \bar{f}\langle\zeta\rangle, \overline{\mathcal{I}}_{\sim}^{\langle\zeta\rangle}\right)$-candidate.

Proof of the Claim. (2) Assume that $r^{*} \in \mathbb{P}_{\zeta}, r^{*} \geq r$, and $\bar{q}^{\prime}=\left\langle q_{\alpha}^{\prime}: \alpha<\lambda\right\rangle$ is a $\mathbb{P}_{\zeta}$-name for an increasing sequence of conditions from $\mathbb{Q}_{\sim} \tilde{\cap} N\left[\tilde{G_{\mathbb{P}_{\zeta}}}\right]$ (so $q_{\alpha}^{\prime}$ is a name for an object in $N\left[G_{\mathbb{P}_{\zeta}}\right]$, but it does not have to belong to $\left.N\right)$. Suppose also that $A_{\xi}$ (for $\xi<\lambda$ ) are $\mathbb{P}_{\zeta}$-names for members of $D \cap \mathbf{V}$.

Construct inductively a sequence $\left\langle r_{i}^{-}, q_{i}^{\prime \prime}, r_{i}, r_{i}^{+}, A_{i}: i<\lambda\right\rangle$ so that

(i) $r_{i}^{-} \in \mathbb{P}_{\zeta} \cap N, r_{i}, r_{i}^{+} \in \mathbb{P}_{\zeta}, r^{*} \leq r_{0}, r_{i}^{-} \leq r_{i} \leq r_{i}^{+}, r_{i}^{-} \leq r_{j}^{-}$for $i<j<\lambda$,

(ii) for each $\varepsilon<\zeta$ and $j<\lambda$ :

$$
r_{j}^{+}\left\lceil\varepsilon \Vdash_{\mathbb{P}_{\varepsilon}} \quad \text { " }\left\langle r_{i}(\varepsilon), r_{i}^{+}(\varepsilon): i \leq j\right\rangle \text { is a legal partial play of } \partial_{0}^{\lambda}\left(\mathbb{Q}_{\varepsilon}\right)\right.
$$

in which Complete uses her regular winning strategy $\mathbf{s t}_{\varepsilon}^{0} "$,

(iii) $q_{i}^{\prime \prime} \in N$ is a $\mathbb{P}_{\zeta}$-name for a condition in $\mathbb{Q}_{\zeta}$ and $r_{i} \Vdash_{\mathbb{P}_{\zeta}}$ " $q_{i}^{\prime}=q_{i}^{\prime \prime}$ ",

(iv) $A_{i} \in D \cap \mathbf{V}$ and $r_{i} \Vdash_{\mathbb{P}_{\zeta}}$ " ${ }_{\sim} A_{i}=A_{i}$ ",

(v) $r_{j}^{-} \Vdash_{\mathbb{P}_{\zeta}}$ " the sequence $\left\langle q_{i}^{\prime \prime}: i \leq j\right\rangle$ is $\leq_{\mathbb{Q}_{\zeta}}$-increasing ".

[Why is the construction possible? First, after arriving to a step $j<\lambda$ we note that the sequence $\left\langle r_{i}, r_{i}^{+}: i<j\right\rangle$ has an upper bound by (ii). So we may choose a condition $r_{j}^{\prime}$ stronger than all $r_{i}, r_{i}^{+}$(for $i<j$ ) and deciding the values of $q_{j}^{\prime}, A_{j}$, say $r_{j}^{\prime} \Vdash_{\mathbb{P}_{\zeta}}$ “ $q_{j}^{\prime}=q_{j}^{\prime \prime} \& \underset{\sim}{A_{j}}=A_{j}$ ", where $q_{j}^{\prime \prime} \in N$ and $A_{j} \in D \cap \mathbf{V}$. Since $r_{j}^{\prime} \geq r_{i}^{-}$ (for all $i<j$ ) and $r_{j}^{\prime} \Vdash_{\mathbb{P}_{\zeta}}$ " the sequence $\left\langle q_{i}^{\prime \prime}: i \leq j\right\rangle$ is increasing ", and since $r_{j}^{\prime}$ is $\left(N, \mathbb{P}_{\zeta}\right)$-generic, we may choose conditions $r_{j}^{-} \in N \cap \mathbb{P}_{\zeta}$ and $r_{j} \in \mathbb{P}_{\zeta}$ so that $r_{i}^{-} \leq r_{j}^{-} \leq r_{j}$ for $i \leq j$ and $r_{j}^{\prime} \leq r_{j}$ and $r_{j}^{-} \mathbb{P}_{\mathbb{P}_{\zeta}}$ " the sequence $\left\langle q_{i}^{\prime \prime}: i \leq j\right\rangle$ is increasing ". Finally, $r_{j}^{+} \in \mathbb{P}_{\zeta}$ is defined essentially by (ii).]

Now define

$$
\underline{q}_{i}^{*}= \begin{cases}\emptyset_{\mathbb{Q}_{\zeta}} & \text { if } i<\Upsilon(\zeta), \\ {\underset{\sim}{q}}_{\alpha}^{\prime \prime} & \text { if } i=\Upsilon(\zeta)+\alpha .\end{cases}
$$

Clearly, $\left\langle r_{i}^{-}\left\langle q_{i}^{*}\right\rangle: i<\lambda\right\rangle$ is an increasing sequence of conditions from $\mathbb{P}_{\zeta^{*}} \cap N$. Therefore, as $\tilde{D}$ is normal and $A_{i} \in D$ and $\bar{f}$ is a $(D, \mathcal{S}, h)$-semi diamond, we may 
ANDRZEJ ROSŁANOWSKI AND SAHARON SHELAH

find a limit ordinal $\delta \in \mathcal{S} \cap \triangle A_{i<\lambda} A_{i}$ such that $\delta>\Upsilon(\zeta) \cdot \omega$ and $\left\langle h \circ f_{\delta}(i): i<\delta\right\rangle=$ $\left\langle r_{i}^{-}\left\langle q_{i}^{*}\right\rangle: i<\delta\right\rangle$. Then for each $\alpha<\delta$ we have

$$
\sim_{\sim}^{\langle\zeta\rangle} \circ f_{\delta}^{\langle\zeta\rangle}(\alpha)={\underset{\sim}{h}}^{\langle\zeta\rangle}\left(f_{\delta}(\Upsilon(\zeta)+\alpha)\right)=h\left(f_{\delta}(\Upsilon(\zeta)+\alpha)\right)(\zeta)=\sim_{\Upsilon}^{*}(\zeta)+\alpha=q_{\alpha}^{\prime \prime} .
$$

Also, $r_{\delta}$ is stronger than all $r_{i}($ for $i<\delta)$ so it forces that $(\forall i<\delta)\left(\underset{\sim}{A_{i}}=A_{i} \& q_{i}^{\prime}=\right.$ $\left.q_{i}^{\prime \prime}\right)$. Hence

$$
r_{\delta} \Vdash " \delta \in \mathcal{S} \cap \underset{\xi<\lambda}{\triangle} \underset{\sim}{A_{\xi}} \quad \& \quad \underset{\sim}{h}\langle\zeta\rangle \circ f_{\delta}^{\langle\zeta\rangle}=\bar{\sim}_{\sim}^{\prime}\lceil\delta " .
$$

The rest should be clear.

\section{Straightforward.}

Remember that $\left\langle\left(\tau_{i}, \zeta_{i}\right): i<\lambda\right\rangle$ was fixed in $(\otimes)_{1},\left\langle w_{i}: i<\lambda\right\rangle$ was chosen in $(\otimes)_{2}$, the function $\Upsilon$ was defined in $(\otimes)_{3}$, and $\bar{q}, p$ are from $(\otimes)_{8}$. Also, $q_{i}^{*}$ are the saturated conditions picked in $(\otimes)_{13}$.

Claim 4.1.4. There is a sequence $\left\langle p_{i}, p_{i}^{+}: i<\lambda\right\rangle \subseteq N$ such that for all $i<\lambda$ we have:

$(\otimes)_{20}^{a}\left(p_{i}, w_{i}\right),\left(p_{i}^{+}, w_{i}\right) \in \mathbb{P}_{\zeta^{*}}^{\mathrm{RS}} \cap N$,

$(\otimes)_{20}^{b}\left(p,\left\{0, \zeta^{*}\right\}\right) \leq^{\prime}\left(p_{j}, w_{j}\right) \leq^{\prime}\left(p_{j}^{+}, w_{j}\right) \leq^{\prime}\left(p_{i}, w_{i}\right)$ for all $j<i$, and if $\varepsilon \in \zeta^{*} \cap N$ and $\Upsilon(\varepsilon)>i$, or $\varepsilon \in \zeta^{*} \backslash N$, and if $G \subseteq \mathbb{P}_{\zeta^{*}}$ is generic over $\mathbf{V}$ such that $\left(p_{i}^{+}, w_{i}\right) \in^{\prime} G$, then

$$
\begin{aligned}
\left(p_{i}^{+}, w_{i}\right)^{G}\left\lceil\varepsilon \Vdash_{\mathbb{P}_{\varepsilon}}\right. & \text { " the sequence }\left\langle\left(p_{j}, w_{j}\right)^{G}(\varepsilon),\left(p_{j}^{+}, w_{j}\right)^{G}(\varepsilon): j \leq i\right\rangle \\
& \text { is a legal partial play of } \partial_{0}^{\lambda}\left(\mathbb{Q}_{\varepsilon}\right) \\
& \text { in which Complete uses her regular strategy } \mathbf{s t}_{\varepsilon}^{0} \text { ", }
\end{aligned}
$$

$(\bigotimes)_{20}^{c}$ if $\varepsilon \in N \cap \zeta^{*}$, then $p_{\Upsilon(\varepsilon)}(\varepsilon)=p_{j}(\varepsilon)=p_{j}^{+}(\varepsilon)$ for all $j \geq \Upsilon(\varepsilon)$,

$(\otimes)_{20}^{d}$ if $i \in \mathcal{S}$ is limit and $\varepsilon \in \operatorname{dom}\left(q_{i}^{*}\right), \Upsilon(\varepsilon) \geq i$, then $p_{i}(\varepsilon)$ is such that for every generic $G \subseteq \mathbb{P}_{\zeta^{*}}$ over $\mathbf{V}$ with $\left(p_{i}, w_{i}\right) \in^{\prime} G$, and two successive members $\varepsilon^{\prime}, \varepsilon^{\prime \prime}$ of the set $w_{i}$ such that $\varepsilon^{\prime} \leq \varepsilon<\varepsilon^{\prime \prime}$ we have:

if $\left\{p_{j}(\varepsilon)\left[G \cap \mathbb{P}_{\varepsilon^{\prime}}\right]\left[G \cap \mathbb{P}_{\varepsilon}\right]: j<i\right\} \cup\left\{q_{i}^{*}(\varepsilon)\left[G \cap \mathbb{P}_{\varepsilon}\right]\right\}$ has an upper bound in $\mathbb{Q}_{\varepsilon}\left[G \cap \mathbb{P}_{\varepsilon}\right]$, then $p_{i}(\varepsilon)\left[G \cap \mathbb{P}_{\varepsilon^{\prime}}\right]\left[G \cap \mathbb{P}_{\varepsilon}\right]$ is such an upper bound,

$(\bigotimes)_{20}^{e}$ for some $\xi \in w_{i+1} \cap \zeta_{i} \backslash\left(\sup \left(w_{i} \cap \zeta_{i}\right)+1\right)$ we have $p_{i+1}\left\lceil\xi=p_{i}^{+}\lceil\xi\right.$ and for some a $\mathbb{P}_{\xi}$-name $\underset{\sim}{\tau} \in N$, for every generic $G \subseteq \mathbb{P}_{\zeta^{*}}$ over $\mathbf{V}$ with $\left(p_{i+1}, w_{i+1}\right) \in^{\prime}$ $G$, we have that $\tau_{i}\left[G \cap \mathbb{P}_{\zeta_{i}}\right]=\tau\left[G \cap \mathbb{P}_{\xi}\right]$.

Proof of the Claim. By induction on $i<\lambda$ we choose $p_{i}, p_{i}^{+}, \mathcal{A}_{i}, \mathcal{A}_{i}^{+}, f_{i}, f_{i}^{+}$such that the following demands $(*)_{1}-(*)_{6}$ are satisfied.

$(*)_{1}\left(p_{i}, w_{i}\right),\left(p_{i}^{+}, w_{i}\right) \in \mathbb{P}_{\zeta^{*}}^{\mathrm{RS}} \cap N$ and $\left(\mathcal{A}_{i}, f_{i}, w_{i}\right),\left(\mathcal{A}_{i}^{+}, f_{i}^{+}, w_{i}\right) \in N$ are their standard representations, respectively, and $\left(p_{0}, w_{0}\right)=\left(p,\left\{0, \zeta^{*}\right\}\right)$.

$(*)_{2}$ If $i<j<\lambda$ then $\left(\mathcal{A}_{i}, f_{i}, w_{i}\right) \preccurlyeq\left(\mathcal{A}_{i}^{+}, f_{i}^{+}, w_{i}\right) \preccurlyeq\left(\mathcal{A}_{j}, f_{j}, w_{j}\right)$.

$(*)_{3}$ If $j<\lambda, \varepsilon \in \zeta^{*} \backslash w_{j}, s_{j} \in \mathcal{A}_{j}^{+}, f_{j}^{+}\left(s_{j}\right) \leq s_{j}$ and for $i \leq j$ the conditions $r_{i} \in \mathcal{A}_{i}, s_{i} \in \mathcal{A}_{i}^{+}$are such that $r_{i} \leq s_{i} \leq s_{j}$, then

$f_{j}^{+}\left(s_{j}\right)\left\lceil\varepsilon \Vdash_{\mathbb{P}_{\varepsilon}} \quad\right.$ " $\left\langle f_{i}\left(r_{i}\right)(\varepsilon), f_{i}^{+}\left(s_{i}\right)(\varepsilon): i \leq j\right\rangle$ is a legal partial play of $\partial_{0}^{\lambda}\left(\mathbb{Q}_{\varepsilon}\right)$ in which Complete uses her regular winning strategy $\mathbf{s t}_{\varepsilon}^{0}$ ". 
$(*)_{4}$ If $\varepsilon \in w_{j}, j<\lambda$ and $r \in \mathcal{A}_{j}, r^{\prime} \in \mathcal{A}_{j}^{+}$and $r^{\prime \prime} \in \mathcal{A}_{\Upsilon(\varepsilon)}$ are such that $r^{\prime \prime} \leq r \leq r^{\prime}$, then

$$
f_{j}(r)(\varepsilon)=f_{j}^{+}\left(r^{\prime}\right)(\varepsilon)=f_{\Upsilon(\varepsilon)}\left(r^{\prime \prime}\right)(\varepsilon) \quad \text { and } \quad p_{j}(\varepsilon)=p_{j}^{+}(\varepsilon)=p_{\Upsilon(\varepsilon)}(\varepsilon) .
$$

$(*)_{5}$ If $j \in \mathcal{S}$ is limit and $\varepsilon \in \operatorname{dom}\left(q_{j}^{*}\right) \backslash \bigcup_{i<j} w_{i}, r \in \mathcal{A}_{j}$ and $r_{i} \in \mathcal{A}_{i}$ are such that $r_{i} \leq r$ for $i<j$ and if $f_{j}(r) \leq r$ then

$\Vdash_{\mathbb{P}_{\varepsilon}}$ " if $\left\{f_{i}\left(r_{i}\right)(\varepsilon): i<j\right\} \cup\left\{q_{j}^{*}(\varepsilon)\right\}$ has an upper bound in $\underset{\sim}{\mathbb{Q}_{\varepsilon}}$ then $f_{j}\left(r_{j}\right)(\varepsilon)$ is such an upper bound ".

$(*)_{6}$ Let $\xi=\sup \left(w_{i+1} \cap \zeta_{i}\right)$. Then $p_{i+1}\left\lceil\xi=p_{i}^{+}\left\lceil\xi\right.\right.$ and for some $\mathbb{P}_{\xi}$-name $\tau \in N$ we have:

if $r \in \mathcal{A}_{i+1}$ and $f_{i+1}(r) \leq r$, then $r \Vdash \underset{\sim}{\tau}=\tau_{\sim}$.

As declared in $(*)_{1}$ we set $p_{0}=p$ and we choose $p_{0}^{+} \in \mathbb{P}_{\zeta^{*}} \cap N$ essentially by $(*)_{3}$. Thus we let $\operatorname{dom}\left(p_{0}^{+}\right)=\operatorname{dom}\left(p_{0}\right), p_{0}(0)=p_{0}^{+}(0)$ and for $\varepsilon \in \operatorname{dom}\left(p_{0}^{+}\right) \backslash\{0\}$ we let $p_{0}^{+}(\varepsilon)$ be the $<_{\chi}^{*}$-first $\mathbb{P}_{\varepsilon}$-name such that

$$
p_{0}^{+}\left\lceil\varepsilon \Vdash " p_{0}^{+}(\varepsilon) \text { is the answer to } p_{0}(\varepsilon) \text { given by the strategy } \mathbf{s t}_{\varepsilon}^{0}\right. \text { ". }
$$

Next we set $\mathcal{A}_{0}=\mathcal{A}_{0}^{+}=\left\{\emptyset_{\mathbb{P}_{\zeta^{*}}}\right\}$ and $f_{0}\left(\emptyset_{\mathbb{P}_{\zeta^{*}}}\right)=p_{0}, f_{0}^{+}\left(\emptyset_{\mathbb{P}_{\zeta^{*}}}\right)=p_{0}^{+}$.

Suppose we have chosen $p_{i}, p_{i}^{+}, \mathcal{A}_{i}, \mathcal{A}_{i}^{+}, f_{i}, f_{i}^{+} \in N$ for $i<j$ so that the relevant demands in $(*)_{1}-(*)_{6}$ are satisfied.

CASE: $\quad j$ is a successor ordinal, say $j=i_{0}+1$.

Let $\xi=\sup \left(w_{j} \cap \zeta_{i_{0}}\right)$ and $\zeta=\min \left(w_{i_{0}} \backslash \zeta_{i_{0}}\right)$. By $(\bigotimes)_{2}^{c}$ we know $\sup \left(w_{i_{0}} \cap \zeta_{i_{0}}\right)<\xi$. Working in $N$ apply Proposition 2.9 to $\xi, \zeta, w_{i_{0}}, w_{j}, \tau_{i_{0}}$ and $\left(p_{i_{0}}^{+}, w_{i_{0}}\right)$ to get $p_{j}$ and $\tau$ such that

$(\otimes)_{1}\left(p_{j}, w_{j}\right) \in \mathbb{P}_{\zeta^{*}}^{\mathrm{RS}} \cap N$ and $\left(p_{i_{0}}^{+}, w_{i_{0}}\right) \leq^{\prime}\left(p_{j}, w_{j}\right)$,

$(\otimes)_{2} p_{j}\left\lceil\left(\xi \cup\left[\zeta, \zeta^{*}\right)\right)=p_{i_{0}}^{+} \uparrow\left(\xi \cup\left[\zeta, \zeta^{*}\right)\right)\right.$,

$(\otimes)_{3} \underset{\sim}{\tau} \in N$ is a $\mathbb{P}_{\xi}$-name,

$(\otimes)_{4}$ if $G \subseteq \mathbb{P}_{\zeta^{*}}$ is generic over $\mathbf{V}$ and $\left(p_{j}, w_{j}\right) \in^{\prime} G$, then $\tau[G]=\tau_{i_{0}}[G]$.

By Proposition 2.17 (used in $N$ ) we may choose a representation $\left(\mathcal{A}_{j}, f_{j}, w_{j}\right) \in N$ for $\left(p_{j}, w_{j}\right)$ such that $\left(\mathcal{A}_{i_{0}}^{+}, f_{i_{0}}^{+}, w_{i_{0}}\right) \preccurlyeq\left(\mathcal{A}_{j}, f_{j}, w_{j}\right)$. Necessarily,

$(\otimes)_{5}$ if $r \in \mathcal{A}_{j}$ and $f_{j}(r) \leq r$, then $r \Vdash \tau \sim \mathcal{\sim}=\tau_{i_{0}}$.

\section{Since}

$(\otimes)_{6}$ if $\Upsilon(\varepsilon)<j$ (i.e., $\varepsilon \in w_{i_{0}}$ ), then either $\varepsilon<\xi$ or $\varepsilon \geq \zeta$,

we have $p_{j}(\varepsilon)=p_{i_{0}}^{+}(\varepsilon)$ whenever $\Upsilon(\varepsilon)<j$. Therefore if $\Upsilon(\varepsilon)<j, r \in \mathcal{A}_{j}$, $r^{+} \in \mathcal{A}_{i_{0}}^{+}, r^{\prime} \in \mathcal{A}_{\Upsilon(\varepsilon)}$ and $r^{\prime \prime} \in \mathcal{A}_{\Upsilon(\varepsilon)}^{+}$are such that $r^{\prime} \leq r^{\prime \prime} \leq r^{+} \leq r$, then $\left(p_{j}(\varepsilon)=p_{\Upsilon(\varepsilon)}^{+}(\varepsilon)=p_{\Upsilon(\varepsilon)}(\varepsilon)\right.$ and $)$

$$
f_{j}(r)(\varepsilon)=f_{i_{0}}^{+}\left(r^{+}\right)(\varepsilon)=f_{\Upsilon(\varepsilon)}^{+}\left(r^{\prime \prime}\right)(\varepsilon)=f_{\Upsilon(\varepsilon)}\left(r^{\prime}\right)(\varepsilon) .
$$

To define $\mathcal{A}_{j}^{+}, f_{j}^{+}$and $p_{j}^{+}$we first note that

$(\otimes)_{7}$ if $r \in \mathcal{A}_{j}, f_{j}(r) \leq r$ and $r_{i} \in \mathcal{A}_{i}, s_{i} \in \mathcal{A}_{i}^{+}$(for $i<j$ ), are such that $r_{i} \leq s_{i} \leq r$, and $\varepsilon \in \zeta^{*} \backslash w_{j}$, then

$$
\begin{aligned}
f_{j}(r) \mid \varepsilon \Vdash_{\mathbb{P}_{\varepsilon}} & \text { " }\left\langle f_{i}\left(r_{i}\right)(\varepsilon), f_{i}^{+}\left(s_{i}\right)(\varepsilon): i<j\right\rangle\left\langle\left\langle f_{j}(r)(\varepsilon)\right\rangle\right. \text { is a partial play of } \\
& \partial_{0}^{\lambda}\left(\mathbb{Q}_{\varepsilon}\right) \text { in which Complete uses her strategy } \mathbf{s}_{\sim}^{0} \mathbf{v}_{\varepsilon}^{0} .
\end{aligned}
$$

Let $r \in \mathcal{A}_{j}$. We define $g(r) \in \mathbb{P}_{\zeta^{*}}$ so that $\operatorname{dom}(g(r))=\operatorname{dom}\left(f_{j}(r)\right)$ and 
$(\otimes)_{8}^{a}$ if $\varepsilon \in \operatorname{dom}(g(r))$ satisfies $\Upsilon(\varepsilon)>j$, then $g(r)(\varepsilon)$ is the $<_{\chi}^{*}$-first $\mathbb{P}_{\varepsilon}$-name for a condition in $\mathbb{Q}_{\varepsilon}$ such that it is forced that

(a) if $\left\langle f_{i}\left(r_{i}\right)(\varepsilon), f_{i}^{+}\left(s_{i}\right)(\varepsilon): i<j\right\rangle\left\langle\left\langle f_{j}(r)(\varepsilon)\right\rangle\right.$ is a legal partial play of $\partial_{0}^{\lambda}\left(\mathbb{Q}_{\varepsilon}\right)$ in which Complete follows her regular winning strategy $\mathbf{s t}_{\varepsilon}^{0}$, then $g(r)(\varepsilon)$ is the answer to this partial play given to Complete by $\mathbf{s t}_{\varepsilon}^{0}$

(b) if the assumptions of (a) are not satisfied then $g(r)(\varepsilon)=f_{j}(r)(\varepsilon)$.

$(\otimes)_{8}^{b}$ For $\varepsilon \in \operatorname{dom}(g(r))$ with $\Upsilon(\varepsilon) \leq j$ we set $g(r)(\varepsilon)=f_{j}(r)(\varepsilon)$.

Clearly, $g$ is a well defined function from $\mathcal{A}_{j}$ to $\mathbb{P}_{\zeta^{*}}$ and $g \in N$. We note that $g(r) \geq f_{j}(r)$ for $r \in \mathcal{A}_{j}$ and

$(\otimes)_{9}$ if $\alpha<\beta$ are two successive elements of $w_{j}, r^{0}, r^{1} \in \mathcal{A}_{j}$ are such that $r^{0}\lceil\alpha$ and $r^{1}\left\lceil\alpha\right.$ are compatible, then $g\left(r^{0}\right)\left\lceil\beta=g\left(r^{1}\right)\lceil\beta\right.$.

[Why? Let $r_{i}^{\ell,+} \in \mathcal{A}_{i}^{+}, r_{i}^{\ell} \in \mathcal{A}_{i}$ for $i<j$ be such that $r_{i}^{\ell} \leq r_{i}^{\ell,+} \leq r^{\ell}$. Then $r_{i}^{0}\lceil\alpha$ and $r_{i}^{1}\left\lceil\alpha\right.$ are compatible and so are $r_{i}^{0,+}\left\lceil\alpha\right.$ and $r_{i}^{1,+}\left\lceil\alpha\right.$. Therefore, by $(*)_{1}$ and 2.10(iv), $f_{i}\left(r_{i}^{0}\right)\left\lceil\beta=f_{i}\left(r_{i}^{1}\right)\left\lceil\beta\right.\right.$ and $f_{i}^{+}\left(r_{i}^{0,+}\right)\left\lceil\beta=f_{i}^{+}\left(r_{i}^{1,+}\right)\left\lceil\beta\right.\right.$. Since the definition of $g\left(r^{\ell}\right)(\varepsilon)$ involves only $\left\langle f_{i}\left(r_{i}^{\ell}\right)(\varepsilon), f_{i}^{+}\left(r_{i}^{\ell,+}\right)(\varepsilon): i<j\right\rangle$ and $f_{j}\left(r^{\ell}\right)(\varepsilon), \mathbf{s t}_{\varepsilon}^{0}, \Upsilon$, we conclude that $g\left(r^{0}\right)(\varepsilon)=g\left(r^{1}\right)(\varepsilon)$ for all $\varepsilon<\beta$.]

Pick a maximal antichain $\mathcal{A}_{j}^{+} \in N$ of $\mathbb{P}_{\zeta^{*}}$ such that

$(\otimes)_{10}^{a}\left(\forall s \in \mathcal{A}_{j}^{+}\right)\left(\exists r \in \mathcal{A}_{j}\right)(r \leq s)$,

$(\otimes)_{10}^{b}$ if $s \in \mathcal{A}_{j}^{+}, r \in \mathcal{A}_{j}$ and $r \leq s$, then either $g(r) \leq s$ or for some $\varepsilon \in \operatorname{dom}(s)$ we have $g(r) \mid \varepsilon \leq s\lceil\varepsilon$ and

$$
s\left\lceil\varepsilon \Vdash_{\mathbb{P}_{\varepsilon}} \text { " the conditions } g(r)(\varepsilon), s(\varepsilon) \text { are incompatible in } \mathbb{Q}_{\varepsilon}\right. \text { ". }
$$

Define $f_{j}^{+}: \mathcal{A}_{j}^{+} \longrightarrow \mathbb{P}_{\zeta^{*}}$ by $f_{j}^{+}(s)=g(r)$ where $r \in \mathcal{A}_{j}$ is the unique member satisfying $r \leq s$. Plainly, the function $f_{j}^{+}$belongs to $N$ and it satisfies condition (v) of Observation $2.12\left(\right.$ by $\left.(\otimes)_{9}\right)$. Hence $\left(\mathcal{A}_{j}^{+}, f_{j}^{+}, w_{j}\right)$ is a standard representation of some $\left(p_{j}^{+}, w_{j}\right) \in \mathbb{P}_{\zeta^{*}}^{\mathrm{RS}} \cap N$. Note also that if $s \in \mathcal{A}_{j}^{+}$and $f_{j}^{+}(s) \leq s$, then letting $r \in \mathcal{A}_{j}$ be such that $s \geq r$ we see that $s \geq g(r) \geq f_{j}(r)$. Thus $r, f_{j}(r)$ are compatible and hence $r \geq f_{j}(r)$. This in turn implies (by $\left.(\otimes)_{7}\right)$ that for each $\varepsilon \in \operatorname{dom}(g(r)$ ) with $\Upsilon(\varepsilon)>j$ the condition $f_{j}(r)\left\lceil\varepsilon\right.$ forces the assumptions of the case (a) of $(\otimes)_{8}$. Consequently the demand $(*)_{3}$ (for $\left.f_{j}^{+}(s)\right)$ holds. One easily verifies that also the other relevant demands from $(*)_{1}-(*)_{6}$ are satisfied by $\mathcal{A}_{j}, f_{j}, p_{j}, \mathcal{A}_{j}^{+}, f_{j}^{+}, p_{j}^{+}$.

CASE: $\quad j$ is a limit ordinal.

If $j \in \mathcal{S}$ then let $q^{*}=q_{j}^{*}$, otherwise $q^{*}=\emptyset_{\mathbb{P}_{\zeta^{*}}}$. Let $\mathcal{A}_{j}^{0} \in N$ be a maximal antichain of $\mathbb{P}_{\zeta^{*}}$ such that

$$
\left(\forall s \in \mathcal{A}_{j}^{0}\right)(\forall i<j)\left(\exists r \in \mathcal{A}_{i}\right)(r \leq s) .
$$

We are going to define a function $g: \mathcal{A}_{j}^{0} \longrightarrow \mathbb{P}_{\zeta^{*}}$. Let $r \in \mathcal{A}_{j}^{0}$ and for $i<j$ let $r_{i} \in \mathcal{A}_{i}, s_{i} \in \mathcal{A}_{i}^{+}$be the unique elements such that $r_{i} \leq s_{i} \leq r$. We define $g(r)$ so that $\operatorname{dom}(g(r))=\bigcup_{i<j} \operatorname{dom}\left(f_{i}\left(r_{i}\right)\right) \cup \operatorname{dom}\left(q^{*}\right)$ and

$(\otimes)_{11}^{a}$ if $\varepsilon \in \operatorname{dom}(g(r))$ and $\Upsilon(\varepsilon) \geq j$, then $g(r)(\varepsilon)$ is the $<_{\chi}^{*}$-first $\mathbb{P}_{\varepsilon}$-name for a condition in $\mathbb{Q}_{\varepsilon}$ for which it is forced that

(a) if $\left\{f_{i}\left(r_{i}\right)(\varepsilon): i<j\right\} \cup\left\{q^{*}(\varepsilon)\right\}$ has an upper bound in $\mathbb{Q}_{\varepsilon}$, then $g(r)(\varepsilon)$ is such an upper bound;

(b) if the assumption of (a) is not satisfied but $\left\{f_{i}\left(r_{i}\right)(\varepsilon): i<j\right\}$ has an upper bound in $\mathbb{Q}_{\varepsilon}$, then $g(r)(\varepsilon)$ is such an upper bound; 
(c) otherwise, $g(r)(\varepsilon)=\emptyset_{\mathbb{Q}_{\varepsilon}}$.

$(\otimes)_{11}^{b}$ For $\varepsilon \in \operatorname{dom}(g(r))$ with $\Upsilon(\varepsilon)<j$ we set $g(r)(\varepsilon)=f_{\Upsilon(\varepsilon)}\left(r_{\Upsilon(\varepsilon)}\right)(\varepsilon)$.

Clearly, $g: \mathcal{A}_{j}^{0} \longrightarrow \mathbb{P}_{\zeta^{*}}$ is a well defined function, $g \in N$.

$(\otimes)_{12}$ Assume $r \in \mathcal{A}_{j}^{0}, r_{i} \in \mathcal{A}_{i}, r_{i} \leq r$ for $i<j$.

(a) If $f_{i}\left(r_{i}\right) \leq r_{i}$ for all $i<j$, then $f_{i}\left(r_{i}\right) \leq g(r)$ for all $i<j$.

(b) If $f_{i}\left(r_{i}\right), r_{i}$ are incompatible for some $i<j$, then $r$ and $g(r)$ are incompatible.

[Why? Clause (a) follows immediately from the inductive hypothesis $(*)_{3}$ and the definition of $g$. To show clause (b) assume that $\varepsilon<\zeta^{*}$ and $i_{0}<j$ are such that

- $f_{i}\left(r_{i}\right)\left\lceil\varepsilon \leq r_{i}\lceil\varepsilon\right.$ for all $i<j$, and

- $r_{i_{0}} \mid \varepsilon \Vdash " f_{i_{0}}\left(r_{i_{0}}\right)(\varepsilon), r_{i_{0}}(\varepsilon)$ are incompatible"

(remember 2.10(iii)). By Observation 2.12(vi) for each $i<j$ there is $s_{i} \in \mathcal{A}_{i}$ such that $r_{i}\left\lceil\varepsilon\right.$ and $s_{i}\left\lceil\varepsilon\right.$ are compatible and $f_{i}\left(s_{i}\right) \leq s_{i}$. Then also $f_{i}\left(r_{i}\right) \uparrow(\varepsilon+1)=$ $f_{i}\left(s_{i}\right)\left\lceil(\varepsilon+1) \leq s_{i} \uparrow(\varepsilon+1)\right.$ and for each $\alpha \leq \varepsilon$ satisfying $\Upsilon(\alpha) \geq j$ we have

$$
\begin{aligned}
f_{i}\left(r_{i}\right)\left\lceil\alpha \Vdash_{\mathbb{P}_{\alpha}}\right. & \text { " }\left\langle f_{i^{\prime}}\left(r_{i^{\prime}}\right)(\alpha): i^{\prime}<i\right\rangle \text { are innings of Incomplete in a play of } \\
& \partial_{0}^{\lambda}\left(\mathbb{Q}_{\alpha}\right) \text { in which Complete follows } \mathbf{s}_{\sim}^{0} \text { " }
\end{aligned}
$$

(Note that if $i^{\prime}<i$ and $t \in \mathcal{A}_{i^{\prime}}$ is such that $t \leq s_{i}$, then $t\left\lceil\varepsilon\right.$ and $r_{i^{\prime}}\lceil\varepsilon$ are compatible, so $f_{i^{\prime}}(t)\left\lceil(\varepsilon+1)=f_{i^{\prime}}\left(r_{i^{\prime}}\right)\lceil(\varepsilon+1)\right.$.) Consequently, by induction on $\alpha \leq \varepsilon+1$ we may show that $f_{i}\left(r_{i}\right)\lceil\alpha \leq g(r)\lceil\alpha$ for all $i<j$ and $\alpha \leq \varepsilon$. In particular, $f_{i_{0}}\left(r_{i_{0}}\right)\left\lceil(\varepsilon+1) \leq g(r)\left\lceil(\varepsilon+1)\right.\right.$ and hence, by the choice of $i_{0}$ and $\varepsilon, g(r)$ and $r_{i_{0}}$ are incompatible. Hence also $g(r)$ and $r$ are incompatible.]

In the same way as for $(\otimes)_{9}$ we may argue that

$(\otimes)_{13}$ if $\alpha<\beta$ are two successive elements of $w_{j}, r^{0}, r^{1} \in \mathcal{A}_{j}^{0}$ are such that $r^{0}\lceil\alpha$ and $r^{1}\left\lceil\alpha\right.$ are compatible, then $g\left(r^{0}\right)\left\lceil\beta=g\left(r^{1}\right)\lceil\beta\right.$.

Now pick a maximal antichain $\mathcal{A}_{j} \in N$ of $\mathbb{P}_{\zeta^{*}}$ such that

$(\otimes)_{14}^{a}\left(\forall s \in \mathcal{A}_{j}\right)\left(\exists r \in \mathcal{A}_{j}^{0}\right)(r \leq s)$ and

$(\otimes)_{14}^{b}$ if $s \in \mathcal{A}_{j}, r \in \mathcal{A}_{j}^{0}$ and $r \leq s$ then either $g(r) \leq s$ or for some $\varepsilon \in \operatorname{dom}(s)$ we have $g(r) \mid \varepsilon \leq s\left\lceil\varepsilon\right.$ and $s\left\lceil\varepsilon \Vdash_{\mathbb{P}_{\varepsilon}}\right.$ " $g(r)(\varepsilon), s(\varepsilon)$ are incompatible in $\mathbb{Q}_{\varepsilon}$ ".

Define $f_{j}: \mathcal{A}_{j} \longrightarrow \mathbb{P}_{\zeta^{*}}$ by $f_{j}(s)=g(r)$ where $r \in \mathcal{A}_{j}^{0}$ is the unique member such that $r \leq s$ for $s \in \mathcal{A}_{j}^{0}$. Using $(\otimes)_{12}+(\otimes)_{13}$ and Proposition 2.13 we may easily argue that $\left(\mathcal{A}_{j}, f_{j}, w_{j}\right) \in N$ is a standard representation of some $\left(p_{j}, w_{j}\right) \in \mathbb{P}_{\zeta^{*}}^{\mathrm{RS}} \cap N$ such that $\left(\mathcal{A}_{i}, f_{i}, w_{i}\right) \preccurlyeq\left(\mathcal{A}_{i}^{+}, f_{i}^{+}, w_{i}\right) \preccurlyeq\left(\mathcal{A}_{j}, f_{j}, w_{j}\right)$ for all $i<j$. Finally, exactly as in the successor case $\left((\otimes)_{7}-(\otimes)_{10}\right.$ and later) we define $\mathcal{A}_{j}, f_{j}^{+}$and $p_{j}^{+}$. We easily verify that the demands $(*)_{1}-(*)_{6}$ are satisfied.

This completes the construction of $\left\langle p_{i}, p_{i}^{+}, \mathcal{A}_{i}, \mathcal{A}_{i}^{+}, f_{i}, f_{i}^{+}: i<\lambda\right\rangle$. It should be self evident that the conditions $(*)_{1}-(*)_{6}$ imply the requirements $(\bigotimes)_{20}^{a}-(\bigotimes)_{20}^{e}$.

Recalling Remark 2.7, we define a condition $r \in \mathbb{P}_{\zeta^{*}}$ by declaring that its domain (support) is $\operatorname{dom}(r)=\zeta^{*} \cap N$ and for each $\zeta \in \zeta^{*} \cap N$

$$
\begin{array}{r}
r\left\lceil\zeta \Vdash " r(\zeta) \geq p_{\Upsilon(\zeta)}(\zeta) \text { is generic for } \bar{q}^{\langle\zeta\rangle}\right. \text { over } \\
\quad N\left[G_{\mathbb{P}_{\zeta}}\right], h_{\sim}^{h \zeta\rangle}, \mathbb{Q}_{\zeta}, R_{\sim}^{\mathrm{pr}}, \bar{f}\langle\zeta\rangle, \mathcal{S}, \Upsilon(\zeta) \cdot \omega " .
\end{array}
$$

Claim 4.1.5. For every $\zeta \in\left(\zeta^{*}+1\right) \cap N$, Generic has a winning strategy in the game $\partial_{1}^{\mathcal{S}}\left(r\left\lceil\zeta, N, h^{[\zeta]}, \mathbb{P}_{\zeta}, R^{\text {pr }}\left\lceil\zeta, \bar{f}, \bar{q}^{[\zeta]}\right)\right.\right.$. 
Proof of the Claim. We prove the claim by induction on $\zeta \in\left(\zeta^{*}+1\right) \cap N$, so suppose that $\zeta \in\left(\zeta^{*}+1\right) \cap N$ and we know that for all $\xi \in N \cap \zeta$ the condition $r\lceil\xi$ is generic for $\bar{q}^{[\xi]}$ over $N, h^{[\xi]}, \mathbb{P}_{\xi}, R^{\text {pr }}\lceil\xi, \bar{f}, \mathcal{S}, 1$. Note, the inductive hypothesis implies that:

$(\boxplus)_{0} r(\xi)$ is well-defined for $\xi \in N \cap \zeta$ (by Claim 4.1.3), and so

$(\boxplus)_{1}$ for each $\xi \in N \cap \zeta$ we may fix a $\mathbb{P}_{\xi}$-name $\mathbf{s t}_{\xi}$ such that the condition $r\lceil\xi$ forces that it is a winning strategy of Generic in the game $\partial_{\Upsilon(\xi) \cdot \omega}^{\mathcal{S}}\left(r(\xi), N\left[G_{\mathbb{P}_{\xi}}\right],{\underset{\sim}{h}}^{\langle\xi\rangle}, \mathbb{Q}_{\xi},{\underset{\sim}{\xi}}_{\xi}^{\mathrm{pr}}, \bar{f}^{\langle\xi\rangle}, \bar{\sim}^{\langle\xi\rangle}\right)$, and

$(\boxplus)_{2}\left(p_{i}^{\zeta}, w_{i}^{\zeta}\right) \leq^{\prime}\left(r\lceil\zeta,\{0, \zeta\})\right.$ for all $i<\lambda$, where $p_{i}^{\zeta}=p_{i} \uparrow \zeta$ and $w_{i}^{\zeta}=\left(w_{i} \cap \zeta\right) \cup$ $\{\zeta\}$.

Subclaim 4.1.5.1. Assume that $\left(p_{i}^{*}, v_{i}\right) \in \mathbb{P}_{\zeta}^{\mathrm{RS}} \cap N$ (for $i<\delta<\lambda$ ), and $p^{*} \in$ $\mathbb{P}_{\zeta} \cap N, r^{\prime} \in \mathbb{P}_{\zeta}$ are such that

$$
r\left\lceil\zeta \leq r^{\prime}, \quad p^{*} \leq r^{\prime} \quad \text { and } \quad(\forall j<i<\delta)\left(\left(p_{j}^{*}, v_{j}\right) \leq^{\prime}\left(p_{i}^{*}, v_{i}\right) \leq^{\prime}\left(r^{\prime},\{0, \zeta\}\right)\right) .\right.
$$

Then there are conditions $p^{+} \in N \cap \mathbb{P}_{\zeta}$ and $r^{+} \in \mathbb{P}_{\zeta}$ such that

$$
p^{*} \leq p^{+} \leq r^{+}, \quad r^{\prime} \leq r^{+} \text {and }(\forall i<\delta)\left(\left(p_{i}^{*}, v_{i}\right) \leq^{\prime}\left(p^{+},\{0, \zeta\}\right)\right) .
$$

Proof of the Subclaim. By the inductive hypothesis (of Claim 4.1.5) we know that $r\left\lceil\xi\right.$ is $\left(N, \mathbb{P}_{\xi}\right)$-generic for all $\xi \in \zeta \cap N$. Therefore, if $\zeta$ is a successor or a limit ordinal of cofinality $\geq \lambda$, then we immediately get that $r\left\lceil\zeta\right.$ is $\left(N, \mathbb{P}_{\zeta}\right)$-generic (remember 3.8 $(5)$ and use $\left.(\otimes)_{1}+(\bigotimes)_{20}^{e}\right)$. Hence either $r^{\prime}$ is $\left(N, \mathbb{P}_{\zeta}\right)$-generic or $\zeta$ is a limit ordinal of cofinality $\operatorname{cf}(\zeta)<\lambda$ and for all $\xi<\zeta$ the condition $r^{\prime} \nmid \xi$ is $\left(N, \mathbb{P}_{\xi}\right)$-generic. In either case Lemma 2.8(3) applies.

Remembering that $\bar{\gamma}=\left\langle\gamma_{\alpha}: \alpha<\lambda\right\rangle$ was defined in Context 3.1(4) we set

$$
E_{0}^{\zeta} \stackrel{\text { def }}{=}\left\{\delta<\lambda: \delta=\gamma_{\delta}>0 \text { and } \delta>\Upsilon(\zeta) \text { and }(\forall \alpha<\delta)(\alpha \cdot \omega<\delta)\right\}
$$

(plainly, $E_{0}^{\zeta} \in D$ ).

We will give a strategy st for Generic in $\partial_{1}^{\mathcal{S}}\left(r \mid \zeta, N, h^{[\zeta]}, \mathbb{P}_{\zeta}, R^{\operatorname{pr}}\left\lceil\zeta, \bar{f}, \bar{q}^{[\zeta]}\right)\right.$. Essentially, for each $\varepsilon \in \zeta \cap N$, Generic is going to play a suitable game at the coordinate $\varepsilon$, but at each time she is playing the game on less than $\lambda$ coordinates. Her actions will be described on the intervals $\left[\gamma_{\alpha}, \gamma_{\alpha+1}\right)$ separately. We may assume that, for $\xi \in N \cap \zeta$, the sets $C_{i} \in D^{\mathbf{V}\left[G_{\mathbb{P}_{\xi}}\right]}$ given to Generic by her winning strategy $\mathbf{s t}_{\xi}$ are of the form $\underset{\alpha<\lambda}{\triangle} \underset{\sim}{A}$, where $\underset{\sim}{A} \in D^{\mathbf{V}}$. To keep the "past and future plays" under control, in addition to the innings $\left(r_{i}^{-}, r_{i}, C_{i}\right)$ of the game, Generic will construct aside

$(\boxplus)_{3}^{\alpha} s_{\alpha}, z_{\alpha}, z_{\alpha}^{\diamond}, r_{j}^{*}, r_{j}^{+}, r_{i}^{\ominus}(\varepsilon), r_{i}^{\oplus}(\varepsilon),{\underset{\sim}{i}}_{i}^{\xi}(\varepsilon), \tau_{\sim}, \varepsilon, A_{i, \varepsilon}^{\xi}$ for $j<\gamma_{\alpha+1}, \xi<\lambda$ and $\varepsilon \in$ $N \cap \zeta$ with $\Upsilon(\varepsilon)<\gamma_{\alpha+1}$ and $i$ such that $\Upsilon(\varepsilon)+i<\gamma_{\alpha+1}$.

The primary roles played by these objects are as follows:

- ${\underset{\sim}{i}}_{i}^{\ominus}(\varepsilon), \sim_{i}^{\oplus}(\varepsilon), \underset{\xi<\lambda}{\triangle} \underset{\sim}{A} \dot{\xi}(\varepsilon)$ are the innings in the stage $i$ of the game played at coordinate $\varepsilon$,

- $s_{\alpha} \in \mathbb{P}_{\zeta}$ are conditions deciding ${\underset{\sim}{i}}_{i}^{\ominus}(\varepsilon), A_{i}^{\xi}(\varepsilon)$ and ${\underset{\sim}{i}, \varepsilon}_{j}, A_{i, \varepsilon}^{\xi}$ are the values forced; the conditions $s_{\alpha}$ will be used as $r_{j}$ 's too,

- $r_{j}^{*}, r_{j}^{+} \in \mathbb{P}_{\zeta}$ form a strategic completeness play and are used to guarantee that the sequences $\left\langle r_{j}: j<\gamma_{\alpha}\right\rangle$ have upper bounds, 
- $z_{\alpha}, z_{\alpha}^{\diamond} \in N \cap \mathbb{P}_{\zeta}$ are conditions forming a strategic completeness play and are used to guarantee that the sequences $\left\langle r_{j}^{-}(\varepsilon): j<\gamma_{\alpha}\right\rangle$ (are forced to) have upper bounds.

Thus we require that the following demands $(\boxplus)_{4}-(\boxplus)_{10}$ are satisfied in addition to the rules of the game.

$(\boxplus)_{4} s_{\alpha}, r_{j}^{*}, r_{j}^{+} \in \mathbb{P}_{\zeta}, z_{\alpha}, z_{\alpha}^{\diamond} \in N \cap \mathbb{P}_{\zeta}$, and $r_{j}^{+} \leq s_{\alpha}, r_{i}^{*} \leq r_{i}^{+} \leq r_{j}^{*}$ for all $i<j<\gamma_{\alpha+1}$.

$(\boxplus)_{5}$ If $\gamma_{\alpha} \notin \mathcal{S}$ then $r_{\gamma_{\alpha}} \leq r_{\gamma_{\alpha}}^{*}$ and if $\gamma_{\alpha} \in \mathcal{S}$ then $r_{\gamma_{\alpha}}=r_{\gamma_{\alpha}}^{*}$. Also, $s_{\alpha} \in$ $\bigcap_{i<\gamma_{\alpha+1}} \mathcal{I}_{i}^{[\zeta]}$ and if $\gamma_{\alpha}<j<\gamma_{\alpha+1}$, then $r_{j}=s_{\alpha}$ and

$$
C_{j}=E_{0}^{\zeta} \cap \bigcap\left\{A_{i, \varepsilon}^{\xi}: \xi<\gamma_{\alpha+1} \& \varepsilon \in N \cap \zeta \& \Upsilon(\varepsilon)+i<\gamma_{\alpha+1}\right\} .
$$

$(\boxplus)_{6}$ If $\varepsilon \in \zeta \backslash\left\{\varepsilon^{\prime} \in N \cap \zeta: \Upsilon\left(\varepsilon^{\prime}\right)<\gamma_{\alpha+1}\right\}, j<\gamma_{\alpha+1}$, then

$$
r_{j}^{+}\left\lceil\varepsilon \Vdash_{\mathbb{P}_{\varepsilon}} \quad \text { “ }\left\langle r_{i}^{*}(\varepsilon), r_{i}^{+}(\varepsilon): i \leq j\right\rangle \text { is a legal partial play of } \partial_{0}^{\lambda}\left(\mathbb{Q}_{\varepsilon}\right)\right.
$$
in which Complete uses her regular winning strategy $\mathbf{s t}_{\varepsilon}^{0} "$.

$(\boxplus)_{7}$ If $\varepsilon \in N \cap \zeta$ and $\Upsilon(\varepsilon)+i<\gamma_{\alpha+1}$, then ${\underset{\sim}{i}}_{i}^{\ominus}(\varepsilon)$ is a $\mathbb{P}_{\varepsilon}$-name for a condition in $N\left[G_{\mathbb{P}_{\varepsilon}}\right] \cap \mathbb{Q}_{\varepsilon}, r_{i}^{\oplus}(\varepsilon)$ is a $\mathbb{P}_{\varepsilon}$-name for a condition in $\mathbb{Q}_{\varepsilon}$ and $\underset{\sim}{A}(\varepsilon)$ are $\mathbb{P}_{\varepsilon}$-names for elements of $D \cap \mathbf{V}$ (for $\xi<\lambda$ ) such that

$$
\begin{aligned}
& r\left\lceil\varepsilon \Vdash_{\mathbb{P}_{\varepsilon}} \quad \text { “ }\left\langle{\underset{\sim}{r}}_{i}^{\ominus}(\varepsilon),{\underset{\sim}{i}}_{i}^{\oplus}(\varepsilon), \underset{\xi<\lambda}{\triangle} \underset{\sim}{\underset{\sim}{\xi}}(\varepsilon): \Upsilon(\varepsilon)+i<\gamma_{\alpha+1}\right\rangle\right. \text { is a partial play }
\end{aligned}
$$

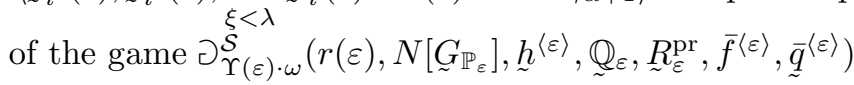

$$
\begin{aligned}
& \text { in which Generic uses her winning strategy } \mathbf{s t}_{\varepsilon} \text { ". }
\end{aligned}
$$

$(\boxplus)_{8}$ If $\varepsilon \in N \cap \zeta$ and $\gamma_{\alpha} \leq j=\Upsilon(\varepsilon)+i<\gamma_{\alpha+1}$, then ${\underset{\sim}{i, \varepsilon}}_{i} \in N$ is a $\mathbb{P}_{\varepsilon}$-name for a condition in $\mathbb{Q}_{\varepsilon}, A_{i, \varepsilon}^{\xi} \in D$ for $\xi<\gamma_{\alpha+1}$, and

$$
s_{\alpha} \mid \varepsilon \Vdash_{\mathbb{P}_{\varepsilon}} \text { “ }{\underset{\sim}{i}}_{i}^{\xi}(\varepsilon)=A_{i, \varepsilon}^{\xi} \text { and if } j \in \mathcal{S} \text { then } r_{j}^{-}(\varepsilon)={\underset{\sim}{r}}_{i}^{\ominus}(\varepsilon)=\tau_{i, \varepsilon} \text { ". }
$$

Also, $r_{j}^{*} \mid \varepsilon \Vdash r_{j}^{*}(\varepsilon)=r_{j}^{+}(\varepsilon)={\underset{\sim}{r}}_{i}^{\oplus}(\varepsilon)$.

$(\boxplus)_{9}$ If $\gamma_{\alpha} \notin \mathcal{S}$, then $\left(p_{j}^{\zeta}, w_{j}^{\zeta}\right) \leq^{\prime}\left(r_{\gamma_{\alpha}+1}^{-},\{0, \zeta\}\right)$ for all $j<\gamma_{\alpha+1}$ (where $p_{j}^{\zeta}=p_{j}\lceil\zeta$ and $w_{j}^{\zeta}=\left(w_{j} \cap \zeta\right) \cup\{\zeta\}$, see $\left.(\boxplus)_{2}\right)$.

$(\boxplus)_{10}$ If $\gamma_{\alpha} \notin \mathcal{S}$, then $r_{\gamma_{\alpha}}^{-} \leq z_{\alpha} \leq z_{\alpha}^{\diamond} \leq r_{\gamma_{\alpha}+1}^{-}$and $z_{\beta}^{\diamond} \leq z_{\alpha}$ for $\beta<\alpha, \gamma_{\beta} \notin \mathcal{S}$, and for each $\varepsilon \in N \cap \zeta$ we have

$$
\begin{aligned}
z_{\alpha}^{\diamond} \mid \varepsilon \Vdash_{\mathbb{P}_{\varepsilon}} & \text { " }\left\langle z_{\beta}(\varepsilon), z_{\beta}^{\diamond}(\varepsilon): \beta \leq \alpha, \gamma_{\beta} \notin \mathcal{S}\right\rangle \text { is a legal partial play of } \\
& \partial_{0}^{\lambda}\left(\mathbb{Q}_{\varepsilon}\right) \text { in which Complete uses her winning strategy } \mathbf{s t}_{\varepsilon}^{0} \text { ". }
\end{aligned}
$$

Suppose that the players arrived to a stage $\gamma_{\alpha}$ of the play and the sequence $\left\langle r_{i}^{-}, r_{i}, C_{i}: i<\gamma_{\alpha}\right\rangle$ has been constructed and the objects listed in $(\boxplus)_{3}^{\beta}$ have been chosen for all $\beta<\alpha$. The procedure applied now by Generic depends on whether the inning at $\gamma_{\alpha}$ is given by Generic or Antigeneric, so we will have two cases below.

CASE 1: $\gamma_{\alpha} \notin \mathcal{S}$.

The inning $\left(r_{\gamma_{\alpha}}^{-}, r_{\gamma_{\alpha}}, C_{\gamma_{\alpha}}\right)$ is chosen by Antigeneric, but we have to argue first that

$(\boxplus)_{11}$ there exists a legal move for Antigeneric. 
For this we note that there is a condition $r^{\prime} \in \mathbb{P}_{\zeta}$ stronger than all $r_{j}$ for $j<\gamma_{\alpha}$. [Why? If $\alpha=\beta+1$ then $s_{\beta}$ works, see $(\boxplus)_{5}$. If $\alpha$ is limit, then look at the conditions $r_{j}^{*}, r_{j}^{+}$for $j<\gamma_{\alpha}$ : by $(\boxplus)_{6}-(\boxplus)_{8}$ the sequence $\left\langle r_{j}^{*}: j<\gamma_{\alpha}\right\rangle$ has an upper bound and by $(\boxplus)_{5}$ this bound will be above all $r_{j}$ for $j<\gamma_{\alpha}$.] Then the condition $r^{\prime}$ is stronger than $r\left\lceil\zeta\right.$ and stronger than all $r_{j}^{-}$(for $j<\gamma_{\alpha}$ ) and we may use Subclaim 4.1.5.1 to pick conditions $r^{-} \in \mathbb{P}_{\zeta} \cap N$ and $r^{*} \in \mathbb{P}_{\zeta}$ so that

$$
r^{-} \leq r^{*}, \quad r^{\prime} \leq r^{*} \text { and }\left(\forall j<\gamma_{\alpha}\right)\left(r_{j}^{-} \leq r^{-}\right) .
$$

Then $\left(r^{-}, r^{*}, \lambda\right)$ is a legal inning of Antigeneric at this stage.

So, let $\left(r_{\gamma_{\alpha}}^{-}, r_{\gamma_{\alpha}}, C_{\gamma_{\alpha}}\right)$ be the inning played by Antigeneric at stage $\gamma_{\alpha}$. By Subclaim 4.1.5.1 Generic may pick conditions $r^{-} \in \mathbb{P}_{\zeta} \cap N$ and $r^{+} \in \mathbb{P}_{\zeta}$ so that

$(\boxplus)_{12} r_{\gamma_{\alpha}}^{-} \leq r^{-} \leq r^{+}, r_{\gamma_{\alpha}} \leq r^{+}$and $\left(\forall j<\gamma_{\alpha+1}\right)\left(\left(p_{j}^{\zeta}, w_{j}^{\zeta}\right) \leq^{\prime}\left(r^{-},\{0, \zeta\}\right)\right)$.

Then $z_{\beta}^{\diamond} \leq r_{\gamma_{\beta}+1}^{-} \leq r_{\gamma_{\alpha}}^{-} \leq r^{-} \leq r^{+}$(for $\beta<\alpha, \gamma_{\beta} \notin \mathcal{S}$ ), so by the argument as in 4.1.5.1 we may apply Lemma 2.5 for $\left\langle z_{\beta}, z_{\beta}^{\diamond}: \beta<\alpha \& \gamma_{\beta} \notin \mathcal{S}\right\rangle$ and $r^{-}, r^{+}$. This will give us conditions $z_{\alpha}, z_{\alpha}^{\diamond} \in N \cap \mathbb{P}_{\zeta}$ and $r_{\gamma_{\alpha}}^{*} \in \mathbb{P}_{\zeta}$ such that $r^{-} \leq z_{\alpha} \leq z_{\alpha}^{\diamond} \leq r_{\gamma_{\alpha}}^{*}$, $r^{+} \leq r_{\gamma_{\alpha}}^{*}$ and for each $\varepsilon \in \zeta \cap N$ the condition $z_{\alpha}^{\diamond}\lceil\varepsilon$ forces that " the sequence $\left\langle z_{\beta}(\varepsilon), z_{\beta}^{\diamond}(\varepsilon): \beta \leq \alpha, \gamma_{\beta} \notin \mathcal{S}\right\rangle$ is a legal partial play of $\partial_{0}^{\lambda}\left(\mathbb{Q}_{\varepsilon}\right)$ in which Complete uses her regular winning strategy st $_{\varepsilon}^{0}$ ". Now, for each $\varepsilon \in N \cap \zeta$ and $i$ with $\Upsilon(\varepsilon)+i<\gamma_{\alpha+1}$ Generic picks ${\underset{\sim}{i}}_{i}^{\ominus}(\varepsilon),{\underset{\sim}{r}}_{i}^{\oplus}(\varepsilon), \sim_{\sim}^{\xi}(\varepsilon)$ so that (for $\Upsilon(\varepsilon)+i<\gamma_{\alpha}$ they are the ones chosen at previous stages and)

$$
\begin{aligned}
& r\left\lceil\varepsilon \Vdash_{\mathbb{P}_{\varepsilon}} \quad “\left\langle{\underset{\sim}{i}}_{i}^{\ominus}(\varepsilon),{\underset{\sim}{r}}_{i}^{\oplus}(\varepsilon), \underset{\xi<\lambda}{\triangle} \underset{\sim}{A} A_{i}^{\xi}(\varepsilon): \Upsilon(\varepsilon)+i<\gamma_{\alpha+1}\right\rangle\right. \text { is a partial play of } \\
& \partial_{\Upsilon(\varepsilon) \cdot \omega}^{\mathcal{S}}\left(r(\varepsilon), N\left[G_{\mathbb{P}_{\varepsilon}}\right], h^{\langle\varepsilon\rangle}, \mathbb{Q}_{\varepsilon},{\underset{\sim}{\varepsilon}}_{\varepsilon}^{\mathrm{pr}}, \bar{f}^{\langle\varepsilon\rangle}, \bar{q}^{\langle\varepsilon\rangle}\right) \\
& \text { in which Generic uses her winning strategy } \mathbf{s t}_{\varepsilon} \text { " }
\end{aligned}
$$

and

- if $\Upsilon(\varepsilon)+i=\gamma_{\alpha}$, theng

$$
r_{\gamma_{\alpha}}^{*}\left\lceil\varepsilon \Vdash_{\mathbb{P}_{\varepsilon}} "{\underset{\sim}{i}}_{i}^{\ominus}(\varepsilon)=z_{\alpha}^{\diamond}(\varepsilon) \&{\underset{\sim}{i}}_{i}^{\oplus}(\varepsilon)=r_{\gamma_{\alpha}}^{*}(\varepsilon) \& \underset{\sim}{A} \underset{i}{\xi}(\varepsilon)=C_{\gamma_{\alpha}} ",\right.
$$

- if $\gamma_{\alpha}<\Upsilon(\varepsilon)<\gamma_{\alpha+1}$, then

$$
r_{\gamma_{\alpha}}^{*}\left\lceil\varepsilon \Vdash_{\mathbb{P}_{\varepsilon}} "{\underset{\sim}{r}}_{0}^{\ominus}(\varepsilon)=z_{\alpha}^{\diamond}(\varepsilon) \&{\underset{\sim}{0}}_{0}^{\oplus}(\varepsilon)=r_{\gamma_{\alpha}}^{*}(\varepsilon) \& \underset{\sim}{A} \underset{0}{\xi}(\varepsilon)=C_{\gamma_{\alpha}} " .\right.
$$

Next, Generic picks $r_{\gamma_{\alpha}}^{+} \in \mathbb{P}_{\zeta}$ so that $\operatorname{dom}\left(r_{\gamma_{\alpha}}^{+}\right)=\operatorname{dom}\left(r_{\gamma_{\alpha}}^{*}\right)$ and the demands of $(\boxplus)_{6}\left(\right.$ for $j=\gamma_{\alpha}$ ) are satisfied and $r_{\gamma_{\alpha}}^{+}(\varepsilon)=r_{\gamma_{\alpha}}^{*}(\varepsilon)$ whenever $\varepsilon \in N \cap \zeta, \Upsilon(\varepsilon)<\gamma_{\alpha+1}$. For $\gamma_{\alpha}<j<\gamma_{\alpha+1}$ she chooses $r_{j}^{*}, r_{j}^{+}$so that $\operatorname{dom}\left(r_{j}^{*}\right)=\operatorname{dom}\left(r_{j}^{+}\right)=\operatorname{dom}\left(r_{\gamma_{\alpha}}^{*}\right)$ and $(\boxplus)_{6}$ holds (for $\varepsilon \in \zeta \backslash\left\{\varepsilon^{\prime} \in N \cap \zeta: \Upsilon\left(\varepsilon^{\prime}\right)<\gamma_{\alpha+1}\right\}$ ) and for $\varepsilon \in N \cap \zeta$ with $\Upsilon(\varepsilon)<\gamma_{\alpha+1}$ we have

- if $j<\Upsilon(\varepsilon)$, then $r_{j}^{*}(\varepsilon)=r_{j}^{+}(\varepsilon)=r_{\gamma_{\alpha}}^{*}(\varepsilon)$, and

- if $j=\Upsilon(\varepsilon)+i$, then $r_{j}^{*}\left\lceil\varepsilon \Vdash\right.$ " $r_{j}^{*}(\varepsilon)=r_{j}^{+}(\varepsilon)={\underset{\sim}{i}}_{i}^{\oplus}(\varepsilon)$ ”.

By $(\boxplus)_{6}$ and $(\boxplus)_{7}$ the sequence $\left\langle r_{j}^{+}: j<\gamma_{\alpha+1}\right\rangle$ has an upper bound in $\mathbb{P}_{\zeta}$, so Generic may choose $s_{\alpha} \in \mathbb{P}_{\zeta}$ and $\tau_{i, \varepsilon} \in N, A_{i, \varepsilon}^{\xi} \in D$ (for $\varepsilon \in N \cap \zeta, \Upsilon(\varepsilon)+i<\gamma_{\alpha+1}$ and $\left.\xi<\gamma_{\alpha+1}\right)$ such that

\footnotetext{
${ }^{9}$ Note that necessarily $i$ is not a successor in this case. Hence if $\gamma_{\alpha}<\Upsilon(\varepsilon) \cdot \omega$, then $i \notin$ $\mathcal{S}[\Upsilon(\varepsilon) \cdot \omega]$ and otherwise $i=\gamma_{\alpha} \notin \mathcal{S}$. Consequently, in the game $\supset_{\Upsilon(\varepsilon) \cdot \omega}^{\mathcal{S}}$ the inning at $i$ belongs to Antigeneric.
} 
- $s_{\alpha}$ is stronger than all $r_{j}^{+}$(for $j<\gamma_{\alpha+1}$ ) and $s_{\alpha} \in \bigcap_{j<\gamma_{\alpha+1}} \mathcal{I}_{j}^{[\zeta]}$, and

- $s_{\alpha}\left\lceil\varepsilon \Vdash_{\mathbb{P}_{\varepsilon}} " r_{i}^{\ominus}(\varepsilon)=\tau_{i, \varepsilon} \& \underset{\sim}{A} A_{i}^{\xi}(\varepsilon)=A_{i, \varepsilon}^{\xi} "\right.$.

She also defines conditions $r_{j}^{-} \in \mathbb{P}_{\zeta} \cap N$ (for $\gamma_{\alpha}<j<\gamma_{\alpha+1}$ ) by declaring that $\operatorname{dom}\left(r_{j}^{-}\right)=\operatorname{dom}\left(z_{\alpha}^{\diamond}\right) \cup\left\{\varepsilon^{\prime} \in N \cap \zeta: \Upsilon\left(\varepsilon^{\prime}\right)<\gamma_{\alpha+1}\right\}$ and

- if $\varepsilon \in \operatorname{dom}\left(r_{j}^{-}\right) \backslash\left\{\varepsilon^{\prime} \in N \cap \zeta: \Upsilon\left(\varepsilon^{\prime}\right)<\gamma_{\alpha+1}\right\}$ or $\varepsilon \in N \cap \zeta, j<\Upsilon(\varepsilon)<\gamma_{\alpha+1}$, then $r_{j}^{-}(\varepsilon)=z_{\alpha}^{\diamond}(\varepsilon)$, and

- if $\varepsilon \in N \cap \zeta$ and $j=\Upsilon(\varepsilon)+i^{*}<\gamma_{\alpha+1}$, then

$r_{j}^{-} \mid \varepsilon \Vdash_{\mathbb{P}_{\varepsilon}} \quad$ "if $\left\langle\tau_{i, \varepsilon}: \gamma_{\alpha} \leq \Upsilon(\varepsilon)+i<\gamma_{\alpha+1}\right\rangle$ is an increasing sequence of conditions above $z_{\alpha}^{\diamond}(\varepsilon)$, then $r_{j}^{-}(\varepsilon)=\tau_{i^{*}, \varepsilon}$, else $r_{j}^{-}(\varepsilon)=z_{\alpha}^{\diamond}(\varepsilon) "$.

Then for $\gamma_{\alpha}<j<\gamma_{\alpha+1}$ Generic plays $r_{j}^{-}$chosen above, $r_{j}=s_{\alpha}$ and $C_{j}=$ $E_{0}^{\zeta} \cap \bigcap\left\{A_{i, \varepsilon}^{\xi}: \xi<\gamma_{\alpha+1} \& \varepsilon \in N \cap \zeta \& \Upsilon(\varepsilon)+i<\gamma_{\alpha+1}\right\}$. Observe that the rules of the game and the demands $(\boxplus)_{4}-(\boxplus)_{10}$ are obeyed by the choices above.

CASE 2: $\quad \gamma_{\alpha} \in \mathcal{S}$ (this may happen only if $\alpha$ is limit).

Generic's choices are similar to those from the previous case, except that $r_{\gamma_{\alpha}}, r_{\gamma_{\alpha}}^{-}$ need to be treated differently, and this influences the choice of $s_{\alpha}$ too.

By $(\boxplus)_{7}$ at previous stages, for each $\varepsilon \in N \cap \zeta$ with $\Upsilon(\varepsilon)<\gamma_{\alpha}$ Generic may pick ${\underset{\sim}{r}}_{i}^{\ominus}(\varepsilon),{\underset{\sim}{i}}_{i}^{\oplus}(\varepsilon)$ and $\underset{\sim}{A_{i}^{\xi}}(\varepsilon)$ (for $\gamma_{\alpha} \leq \Upsilon(\varepsilon)+i<\gamma_{\alpha+1}$ ) so that $(\boxplus)_{7}$ still holds. By $(\boxplus)_{6}+(\boxplus)_{8}$ she may choose a condition $r^{\prime} \in \mathbb{P}_{\zeta}$ stronger than all $r_{j}$ for $j<\gamma_{\alpha}$ and such that $r^{\prime}(\varepsilon)=r_{i}^{\oplus}(\varepsilon)$ when $\varepsilon \in N \cap \zeta, \Upsilon(\varepsilon)+i=\gamma_{\alpha}, 0<i$. Now, for $\varepsilon \in \zeta \cap N$ with $\gamma_{\alpha} \leq \Upsilon(\varepsilon)<\gamma_{\alpha+1}$ we have that

$$
\begin{gathered}
r^{\prime} \mid \varepsilon \Vdash_{\mathbb{P}_{\varepsilon}} \text { " the condition } r^{\prime}(\varepsilon) \text { is }\left(N\left[G_{\mathbb{P}_{\varepsilon}}\right], \mathbb{Q}_{\varepsilon}\right) \text {-generic and } \\
r^{\prime}(\varepsilon) \geq r_{j}^{-}(\varepsilon) \text { for all } j<\gamma_{\alpha} " .
\end{gathered}
$$

Therefore, by Observation 2.3, Generic may pick $\mathbb{P}_{\varepsilon}-$ names ${\underset{\sim}{0}}_{0}^{\ominus}(\varepsilon),{ }_{\sim}^{\oplus}(\varepsilon)$ for conditions in $\mathbb{Q}_{\varepsilon}$ such that

$$
r \mid \varepsilon \Vdash_{\mathbb{P}_{\varepsilon}} \text { “ }{\underset{\sim}{0}}_{0}^{\ominus}(\varepsilon) \in N\left[G_{\mathbb{P}_{\varepsilon}}\right] \&{\underset{\sim}{0}}_{0}^{\ominus}(\varepsilon) \leq{\underset{\sim}{r}}_{0}^{\oplus}(\varepsilon) \& r(\varepsilon) \leq{\underset{\sim}{r}}_{0}^{\oplus}(\varepsilon) ",
$$

and

$$
r^{\prime} \mid \varepsilon \Vdash_{\mathbb{P}_{\varepsilon}} " r^{\prime}(\varepsilon) \leq{\underset{\sim}{r}}_{0}^{\oplus}(\varepsilon) \&\left(\forall j<\gamma_{\alpha}\right)\left(r_{j}^{-}(\varepsilon) \leq \sim_{0}^{\ominus}(\varepsilon)\right) " .
$$

Then for $\varepsilon \in \zeta \cap N, \xi<\lambda$ and $i$ such that $\gamma_{\alpha} \leq \Upsilon(\varepsilon)<\Upsilon(\varepsilon)+i<\gamma_{\alpha+1}$ Generic picks $\mathbb{P}_{\varepsilon}$-names ${\underset{\sim}{r}}_{i}^{\ominus}(\varepsilon),{\underset{\sim}{i}}_{i}^{\oplus}(\varepsilon)$ and $\underset{\sim}{A} \dot{\xi}(\varepsilon)$ so that $(\boxplus)_{7}$ holds.

Now she declares that $\operatorname{dom}\left(r_{\gamma_{\alpha}}\right)=\operatorname{dom}\left(r_{\gamma_{\alpha}}^{*}\right)=\operatorname{dom}\left(r^{\prime}\right)$ and for $\varepsilon \in \operatorname{dom}\left(r_{\gamma_{\alpha}}\right)$ she sets

$$
r_{\gamma_{\alpha}}(\varepsilon)=r_{\gamma_{\alpha}}^{*}(\varepsilon)= \begin{cases}\underset{r_{0}}{r^{\oplus}}(\varepsilon) & \text { if } \varepsilon \in N \cap \zeta, \gamma_{\alpha} \leq \Upsilon(\varepsilon)<\gamma_{\alpha+1}, \\ r^{\prime}(\varepsilon) & \text { otherwise. }\end{cases}
$$

Then $r_{\gamma_{\alpha}}^{+}, r_{j}^{*}, r_{j}^{+}$(for $\gamma_{\alpha}<j<\gamma_{\alpha+1}$ ) are defined exactly as in the previous case. Like before, the sequence $\left\langle r_{j}^{+}: j<\gamma_{\alpha+1}\right\rangle$ has an upper bound in $\mathbb{P}_{\zeta}$, so Generic may find a condition $s^{\prime} \in \mathbb{P}_{\zeta}$, names $\tau_{i, \varepsilon} \in N$ for conditions in $\mathbb{Q}_{\varepsilon}$ and sets $A_{i, \varepsilon}^{\xi} \in D$ (for $\varepsilon \in N \cap \zeta, \gamma_{\alpha} \leq \Upsilon(\varepsilon)+i<\gamma_{\alpha+1}$ and $\left.\xi<\gamma_{\alpha+1}\right)$ such that

- $r_{j}^{+} \leq s^{\prime}$ for all $j<\gamma_{\alpha+1}$ and $s^{\prime} \in \bigcap_{j<\gamma_{\alpha+1}} \mathcal{I}_{j}^{[\zeta]}$, and - $s^{\prime} \mid \varepsilon \Vdash_{\mathbb{P}_{\varepsilon}} " \underset{\sim}{r_{i}^{\ominus}}(\varepsilon)=\tau_{i, \varepsilon} \& \underset{\sim}{A}(\varepsilon)=A_{i, \varepsilon}^{\xi}$ ". 
Clearly $r_{j}^{-} \leq r_{j} \leq r^{\prime} \leq r_{\gamma_{\alpha}} \leq s^{\prime}$ for $j<\gamma_{\alpha}$, so Generic may use Subclaim 4.1.5.1 to pick conditions $s_{\alpha} \in \mathbb{P}_{\zeta}$ and $r^{-} \in N \cap \mathbb{P}_{\zeta}$ such that $r^{-} \leq s_{\alpha}, s^{\prime} \leq s_{\alpha}$ and $\left(\forall j<\gamma_{\alpha}\right)\left(r_{j}^{-} \leq r^{-}\right)$. Moreover, Generic may modify $r^{-}$so that, additionally, for each $\varepsilon \in \operatorname{dom}\left(r^{-}\right)$we have

$\Vdash_{\mathbb{P}_{\varepsilon}} \quad$ "if the sequence $\left\langle r_{j}^{-}(\varepsilon): j<\gamma_{\alpha}\right\rangle$ has an upper bound in $\mathbb{Q}_{\varepsilon}$ then $r^{-}(\varepsilon)$ is such a bound ".

Then, for $\gamma_{\alpha} \leq j<\gamma_{\alpha+1}$, Generic sets $\operatorname{dom}\left(r_{j}^{-}\right)=\operatorname{dom}\left(r^{-}\right)$and

- if $\varepsilon \in \operatorname{dom}\left(r_{j}^{-}\right) \backslash\left\{\varepsilon^{\prime} \in N \cap \zeta: \Upsilon\left(\varepsilon^{\prime}\right)<\gamma_{\alpha+1}\right\}$, then $r_{j}^{-}(\varepsilon)=r^{-}(\varepsilon)$, and

- if $\varepsilon \in N \cap \zeta, \Upsilon(\varepsilon)<\gamma_{\alpha+1}$ and $i^{*}$ is 0 if $j \leq \Upsilon(\varepsilon)$ and $i^{*}$ is such that $j=\Upsilon(\varepsilon)+i^{*}$ if $\Upsilon(\varepsilon)<j<\gamma_{\alpha+1}$, then $r_{j}^{-}(\varepsilon)$ is the $<_{\chi}^{*}$-first name for a condition in $\mathbb{Q}_{\varepsilon}$ such that

$r_{j}^{-} \mid \varepsilon \Vdash_{\mathbb{P}_{\varepsilon}} \quad$ "if $\left\langle\tau_{i, \varepsilon}: \gamma_{\alpha} \leq \Upsilon(\varepsilon)+i<\gamma_{\alpha+1}\right\rangle$ is an increasing sequence of conditions, and $\tau_{0, \varepsilon}$ is above all $r_{j^{\prime}}^{-}(\varepsilon)$ for $j^{\prime}<\gamma_{\alpha}$, then $r_{j}^{-}(\varepsilon)=\tau_{i^{*}, \varepsilon}, \quad$ and else $r_{j}^{-}(\varepsilon)=r^{-}(\varepsilon)$ ".

Since in the current case $\alpha$ is a limit ordinal, $(\boxplus)_{10}$ implies that for each $\varepsilon \in N \cap \zeta$ and a condition $z \in \mathbb{P}_{\varepsilon}$ stronger than all $r_{j^{\prime}}^{-} \mid \varepsilon$ (for $j^{\prime}<\gamma_{\alpha}$ ) we have

$z \Vdash_{\mathbb{P}_{\varepsilon}}$ " the sequence $\left\langle r_{j^{\prime}}^{-}(\varepsilon): j^{\prime}<\gamma_{\alpha}\right\rangle$ has an upper bound in $\underset{\sim}{\mathbb{Q}}$ ",

and thus $z \Vdash_{\mathbb{P}_{\varepsilon}} " r_{j^{\prime}}^{-}(\varepsilon) \leq r^{-}(\varepsilon)$ for all $j^{\prime}<\gamma_{\alpha}$ " (remember the additional demand on $r^{-}$). Consequently, the conditions $r_{j}^{-}$(for $\gamma_{\alpha} \leq j<\gamma_{\alpha+1}$ ) are well defined, they belong to $N$ and if $j^{\prime}<\gamma_{\alpha}$ and $\gamma_{\alpha} \leq j_{1}<j_{2}<\gamma_{\alpha+1}$ then $r_{j^{\prime}}^{-} \leq r_{j_{1}}^{-} \leq r_{j_{2}}^{-} \leq s_{\alpha}$. Finally, for $\gamma_{\alpha} \leq j<\gamma_{\alpha+1}$ Generic plays $r_{j}^{-}$chosen above, $r_{\gamma_{\alpha}}$ and $r_{j}=s_{\alpha}$ if $j>\gamma_{\alpha}$, and $C_{j}=E_{0}^{\zeta} \cap \bigcap\left\{A_{i, \varepsilon}^{\xi}: \xi<\gamma_{\alpha+1} \& \varepsilon \in N \cap \zeta \& \Upsilon(\varepsilon)+i<\gamma_{\alpha+1}\right\}$. Easily the rules of the gam 10 , and the demands $(\boxplus)_{4^{-}}(\boxplus)_{10}$ are obeyed by the choices above.

Subclaim 4.1.5.2. The strategy $\mathbf{s t}$ described above is a winning strategy for Generic in $\partial_{1}^{\mathcal{S}}\left(r\left\lceil\zeta, N\left[G_{\mathbb{P}_{\zeta}}\right], h[\zeta], \mathbb{P}_{\zeta}, R^{\operatorname{pr}} \zeta \zeta, \bar{f}, \bar{q}^{[\zeta]}\right)\right.$.

Proof of the Subclaim. Suppose that $\left\langle r_{j}^{-}, r_{j}, C_{j}: j<\lambda\right\rangle$ is a result of a play of $\partial_{1}^{\mathcal{S}}\left(r \uparrow \zeta, N\left[G_{\mathbb{P}_{\zeta}}\right], h^{[\zeta]}, \mathbb{P}_{\zeta}, R^{\text {pr }}\left\lceil\zeta, \bar{f}, \bar{q}^{[\zeta]}\right)\right.$ in which Generic follows the strategy st. By what was said in the description of the strategy (specifically in $(\boxplus)_{11}$ ) both players had always legal moves, so the play lasted really $\lambda$ steps. Let

$$
s_{\alpha}, z_{\alpha}, z_{\alpha}^{\diamond}, r_{j}^{*}, r_{j}^{+}, r_{i}^{\ominus}(\varepsilon), r_{i}^{\oplus}(\varepsilon), A_{\sim}^{\xi}(\varepsilon), \tau_{i, \varepsilon}, A_{i, \varepsilon}^{\xi}
$$

be the objects written aside by Generic (so they satisfy the demands $(\boxplus)_{4}-(\boxplus)_{10}$ ).

We will argue that condition $(\circledast)$ of Definition 3.5(3) holds (i.e., Generic wins).

Assume that a limit ordinal $\delta \in \mathcal{S} \cap \bigcap_{j<\delta} C_{j}$ is such that $h^{[\zeta]}\left(f_{\delta}(j)\right)=r_{j}^{-}$for each successor $j<\delta$. Then also $\delta \in E_{0}^{\zeta}$, so $(\forall \alpha<\delta)(\alpha \cdot \omega<\delta)$ and $\Upsilon(\zeta)<\delta=$ $\gamma_{\delta}=\sup \left(\gamma_{\alpha}: \alpha<\delta \& \gamma_{\alpha} \notin \mathcal{S}\right)$. Therefore, by $(\boxplus)_{9}$, we have that

$$
(\forall i<\delta)(\exists j<\delta)\left(\left(p_{i}^{\zeta}, w_{i}^{\zeta}\right) \leq^{\prime}\left(r_{j}^{-},\{0, \zeta\}\right)\right),
$$

\footnotetext{
${ }^{10}$ Note: in this case it is not required that $r_{\gamma_{\alpha}} \leq r_{\gamma_{\alpha}}$ as $\gamma_{\alpha} \in \mathcal{S} \cap \mathcal{R}$.

${ }^{11}$ Note: $h^{[\zeta]} \circ f_{\delta}$ is an increasing sequence of conditions by $(\otimes)_{9}$
} 
and consequently if $\zeta^{\prime}=\min \left(\zeta_{\delta}^{*}, \zeta\right)$, then $\left(p_{i}^{\zeta^{\prime}}, w_{i}^{\zeta^{\prime}}\right) \leq^{\prime}\left(q_{\delta}^{*} \mid \zeta^{\prime},\left\{0, \zeta^{\prime}\right\}\right)$ for all $i<\delta$. (Remember, $q_{\delta}^{*}$ is an upper bound to $h^{\left[\zeta_{\delta}^{*}\right]} \circ f_{\delta}$, see $(\otimes)_{12}+(\otimes)_{13}$.) Hence, by $(\boxplus)_{20}^{d}+(\boxplus)_{2}$,

$(\boxplus)_{13}$ if $\varepsilon \in \zeta \cap \zeta_{\delta}^{*} \cap N, \Upsilon(\varepsilon) \geq \delta$ and $z \in \mathbb{P}_{\varepsilon}$ is stronger than both $q_{\delta}^{*}\lceil\varepsilon$ and $r\lceil\varepsilon$, then $z \Vdash q_{\delta}^{*}(\varepsilon) \leq r(\varepsilon)$.

Let $\bar{\beta}=\left\langle\beta(\varepsilon) \leq \varepsilon^{*}\right\rangle$ be the increasing (continuous) enumeration of $w_{\delta} \cap(\zeta+1)$. Then $\beta(0)=0 \in w_{0}$ and $\beta\left(\varepsilon^{*}\right)=\zeta \in w_{i^{*}}$, where $i^{*}=\Upsilon(\zeta)<\delta$. Since $r_{\delta}$ is stronger than all $r_{j}^{-}, r_{j}$ (for $j<\delta$ ) we also have that for each $\varepsilon \leq \varepsilon^{*}$ :

$(\boxplus)_{14}^{a} r_{\delta}\left\lceil\beta(\varepsilon)\right.$ is an upper bound to $h^{[\beta(\varepsilon)]} \circ f_{\delta}$, and

$(\boxplus)_{14}^{b} r_{\delta}\left\lceil\beta(\varepsilon) \in \bigcap_{\alpha<\delta} \mathcal{I}_{\alpha}^{[\beta(\varepsilon)]}\left(\right.\right.$ by $\left.(\boxplus)_{5}\right)$, and

$(\boxplus)_{14}^{c}$ if $\beta(\varepsilon) \in w_{i} \cap \zeta$ for some $i<\delta$, then $\Upsilon(\beta(\varepsilon)) \cdot \omega<\delta$ and $\delta \in \bigcap\left\{A_{j, \beta(\varepsilon)}^{\xi}\right.$ : $\xi, j<\delta\}$, and hence (by $\left.(\boxplus)_{8}\right)$ the condition $r_{\delta}\left\lceil\beta(\varepsilon)\right.$ forces $\left(\right.$ in $\left.\mathbb{P}_{\beta(\varepsilon)}\right)$ that:

$\delta \in \underset{j<\lambda}{\triangle} \underset{\xi<\lambda}{A_{\sim}^{\xi}}(\beta(\varepsilon)) \cap \mathcal{S}[\Upsilon(\beta(\varepsilon)) \cdot \omega]$ and $\stackrel{\sim}{h}^{\langle\beta(\varepsilon)\rangle} \circ f_{\delta}^{\langle\beta(\varepsilon)\rangle}$ is an increasing sequence of conditions in $\mathbb{Q}_{\beta(\varepsilon)}$ and $\stackrel{\sim}{h}^{\langle\beta(\varepsilon)\rangle}\left(f_{\delta}^{\langle\beta(\varepsilon)\rangle}(j)\right)=h\left(f_{\delta}(\Upsilon(\beta(\varepsilon))+j)\right)(\beta(\varepsilon))=r_{\Upsilon(\beta(\varepsilon))+j}^{-}(\beta(\varepsilon))=$ $\underline{\sim}_{j}^{\ominus}(\beta(\varepsilon))$ for all successor $j<\delta$.

By induction on $\varepsilon \leq \varepsilon^{*}$ we are going to show that

$(\boxplus)_{15}^{a}\left(h^{[\beta(\varepsilon)]} \circ f_{\delta}\right)\left(R^{\operatorname{pr}}\lceil\beta(\varepsilon))\left(r_{\delta}\lceil\beta(\varepsilon))\right.\right.$, and

$(\boxplus)_{15}^{b} \beta(\varepsilon) \leq \zeta_{\delta}^{*}$ and $q_{\delta}^{[\beta(\varepsilon)]}=q_{\delta}^{*}\left\lceil\beta(\varepsilon) \leq r_{\delta}\lceil\beta(\varepsilon)\right.$.

To start we note that $\left\langle{\underset{\sim}{j}}_{j}^{\ominus}(0), \underset{\sim}{\underset{\sim}{\oplus}}(0), \underset{\xi<\lambda}{\triangle} \underset{\sim}{A} A_{j}^{\xi}(0): j<\delta\right\rangle$ is a legal partial play of $\supset_{0}^{\mathcal{S}}\left(r(0), N, \underset{\sim}{h}\langle 0\rangle, \mathbb{Q}_{0},{\underset{\sim}{0}}_{0}^{\mathrm{pr}}, \bar{f}^{\langle 0\rangle}, \bar{\sim}^{\langle 0\rangle}\right)$ in which Generic uses her winning strategy $\mathbf{s t}_{0}$ (and all names involved are actually objects from $\mathbf{V}$ ). Also $\delta \in \underset{j<\lambda}{\triangle} \underset{\xi<\lambda}{\underset{\sim}{A}} A_{j}^{\xi}(0)$, ${\underset{\sim}{j}}_{j}^{\ominus}(0)=r_{j}^{-}(0)$ for all $j \in \delta \cap \mathcal{S}$ (by $\left.(\boxplus)_{8}\right)$ and $r_{j}^{-}(0)={\underset{\sim}{h}}^{\langle 0\rangle}\left(f_{\delta}(j)\right)$ for all successor $j<\delta$. Therefore, as the play on the coordinate $\varepsilon=0$ is won by Generic, $r_{\delta}(0)=$ $r_{\delta}^{*}(0)={\underset{\sim}{\delta}}_{\delta}^{\oplus}(0) \geq q_{\delta}^{\langle 0\rangle}$ and $\left({\underset{\sim}{h}}^{\langle 0\rangle} \circ f_{\delta}\right){\underset{\sim}{R}}_{0}^{\mathrm{pr}} r_{\delta}(0)$. Also, by $(\boxplus)_{14}^{a}, r_{\delta}\lceil\beta(1)$ is an upper bound to $h^{[\tilde{\beta(1)]}} \circ f_{\delta}$ and hence $\left(h^{[\beta(1)]} \circ f_{\delta}\right)\left(R^{\text {pr }} \uparrow \beta(1)\right)\left(r_{\delta}\lceil\beta(1))\right.$. And since $r_{\delta}\left\lceil\beta(1) \in \bigcap_{\alpha<\delta} \mathcal{I}_{\alpha}^{[\beta(1)]}\right.$ (see $(\boxplus)_{14}^{b}$ ) we may conclude that $\zeta_{\delta}^{*} \geq \beta(1)$ and therefore $q_{\delta}^{[\beta(1)]}=q_{\delta}^{*}\left\lceil\beta(1)\right.$ (as $\beta(1)$ is not a limit of members of $w_{\delta}$ ). Now by induction on $\xi \leq \beta(1)$ we argue that $q_{\delta}^{*}\left\lceil\xi \leq r_{\delta}\left\lceil\xi\right.\right.$ : it follows from $(\otimes)_{19}$ and what has been said above that $q_{\delta}^{*}(0)=q_{\delta}^{\langle 0\rangle} \leq r_{\delta}(0)$. Suppose $0<\xi<\beta(1), \xi \in N$, and assume that we have shown $q_{\delta}^{*} \mid \xi \leq r_{\delta}\left\lceil\xi\right.$. Then, by $(\boxplus)_{13}, r_{\delta}\left\lceil\xi \Vdash_{\mathbb{P}_{\xi}} q_{\delta}^{*}(\xi) \leq r(\xi) \leq r_{\delta}(\xi)\right.$. This concludes the arguments for $(\boxplus)_{15}$ when $\varepsilon=1$.

Suppose we have justified $(\boxplus)_{15}$ for $\varepsilon_{0}<\varepsilon^{*}$ and $\Upsilon\left(\beta\left(\varepsilon_{0}\right)\right)<\delta$. By $(\boxplus)_{7}$, the condition $r_{\delta}\left\lceil\beta\left(\varepsilon_{0}\right)\right.$ forces that

$$
\left\langle\sim_{i}^{\ominus}\left(\beta\left(\varepsilon_{0}\right)\right),{\underset{\sim}{i}}_{i}^{\oplus}\left(\beta\left(\varepsilon_{0}\right)\right), \underset{\xi<\lambda}{\triangle} \underset{\sim}{A}{ }_{i}^{\xi}\left(\beta\left(\varepsilon_{0}\right)\right): \Upsilon\left(\beta\left(\varepsilon_{0}\right)\right)+i<\delta\right\rangle
$$

is a legal partial play of the game

$$
\partial_{\Upsilon\left(\varepsilon\left(\beta_{0}\right)\right)}^{\mathcal{S}}\left(r\left(\beta\left(\varepsilon_{0}\right)\right), N\left[G_{\mathbb{P}_{\beta\left(\varepsilon_{0}\right)}}\right], h_{\sim}^{\left\langle\beta\left(\varepsilon_{0}\right)\right\rangle}, \mathbb{Q}_{\beta\left(\varepsilon_{0}\right)}, R_{\beta\left(\varepsilon_{0}\right)}^{\mathrm{pr}}, \bar{f}^{\left\langle\beta\left(\varepsilon_{0}\right)\right\rangle}, \bar{\sim}^{\left\langle\beta\left(\varepsilon_{0}\right)\right\rangle}\right)
$$


in which Generic uses the winning strategy $\mathbf{s t}_{\sim\left(\varepsilon_{0}\right)}$. Therefore, using $(\boxplus)_{14}^{c}$ and $(\boxplus)_{5}+(\boxplus)_{8}$, we get that $r_{\delta}\left\lceil\beta\left(\varepsilon_{0}\right)\right.$ forces (in $\mathbb{P}_{\beta\left(\varepsilon_{0}\right)}$ ) that

$$
\text { “ } r_{\delta}\left(\beta\left(\varepsilon_{0}\right)\right)={\underset{\sim}{\delta}}_{\delta}^{\oplus}\left(\beta\left(\varepsilon_{0}\right)\right) \geq \underset{\sim}{q_{\delta}}\left\langle\beta\left(\varepsilon_{0}\right)\right\rangle \text { and }\left({\underset{\sim}{h}}^{\left\langle\beta\left(\varepsilon_{0}\right)\right\rangle} \circ f_{\delta}^{\left\langle\beta\left(\varepsilon_{0}\right)\right\rangle}\right) \underset{\sim}{R_{\beta\left(\varepsilon_{0}\right)}^{\mathrm{pr}}} r_{\delta}\left(\beta\left(\varepsilon_{0}\right)\right) " .
$$

Since $\underset{\sim}{h}\left\langle\beta\left(\varepsilon_{0}\right)\right\rangle \circ f_{\delta}^{\left\langle\beta\left(\varepsilon_{0}\right)\right\rangle}(j)=h\left(f_{\delta}\left(\Upsilon\left(\beta\left(\varepsilon_{0}\right)\right)+j\right)\left(\beta\left(\varepsilon_{0}\right)\right)\right.$ and $r_{\delta}\left\lceil\beta\left(\varepsilon_{0}+1\right)\right.$ is an upper bound to $h^{\left[\beta\left(\varepsilon_{0}\right)\right]} \circ f_{\delta}$ (by $\left.(\boxplus)_{14}^{a}\right)$, we may use our inductive hypothesis of $(\boxplus)_{15}^{a}$ for $\varepsilon_{0}$ to conclude that $\left(h^{\left[\beta\left(\varepsilon_{0}+1\right)\right]} \circ f_{\delta}\right)\left(R^{\mathrm{pr}}\left\lceil\beta\left(\varepsilon_{0}+1\right)\right)\left(r_{\delta}\left\lceil\beta\left(\varepsilon_{0}\right)\right)\right.\right.$ (i.e., $(\boxplus)_{15}^{a}$ for $\left.\varepsilon=\varepsilon_{0}+1\right)$. By $(\boxplus)_{14}^{b}$ and the inductive hypothesis of $(\boxplus)_{15}^{b}$ for $\varepsilon_{0}$ we see now that $\zeta_{\delta}^{*} \geq \beta\left(\varepsilon_{0}+1\right)$ and also, as $\beta\left(\varepsilon_{0}+1\right)$ is not the limit of elements of $w_{\delta}$, $q_{\delta}^{\left[\beta\left(\varepsilon_{0}+1\right)\right]}=q_{\delta}^{*}\left\lceil\beta\left(\varepsilon_{0}+1\right)\right.$. Like before we may use $(\boxplus)_{13}+(\otimes)_{19}$ to show inductively that for all $\xi \leq \beta\left(\varepsilon_{0}+1\right)$ we have $q_{\delta}^{*}\left|\xi \leq r_{\delta}\right| \xi$, getting $(\boxplus)_{15}^{b}$ for $\varepsilon=\varepsilon_{0}+1$.

Suppose that we have shown $(\boxplus)_{15}$ for $\varepsilon_{0}<\varepsilon^{*}$ and $\Upsilon\left(\beta\left(\varepsilon_{0}\right)\right)=\delta$ (so $\beta\left(\varepsilon_{0}\right)$ is the limit of points from $\left.\bigcup_{i<\delta} w_{i}\right)$. The assumption of $(\boxplus)_{15}^{a}$ for $\varepsilon_{0}$ implies immediately that $\left(h^{\left[\beta\left(\varepsilon_{0}+1\right)\right]} \circ f_{\delta}\right)\left(R^{\text {pr }} \uparrow \beta\left(\varepsilon_{0}+1\right)\right)\left(r_{\delta}\left\lceil\beta\left(\varepsilon_{0}\right)\right)\right.$ as there are no new "active" coordinates here (remember $(\otimes)_{4}^{b}$ and $\left.(\boxplus)_{14}^{a}\right)$. The same way as before we argue that $(\boxplus)_{15}^{b}$ holds for $\varepsilon=\varepsilon_{0}+1$ as well.

Now suppose that $\varepsilon \leq \varepsilon^{*}$ is a limit ordinal and that we have shown $(\boxplus)_{15}$ for all $\varepsilon^{\prime}<\varepsilon$. Since $\beta(\varepsilon)=\sup \left(\beta\left(\varepsilon^{\prime}\right): \varepsilon^{\prime}<\varepsilon\right)$, the assumption of $(\boxplus)_{15}^{a}$ for $\varepsilon^{\prime}<\varepsilon$ implies that $\left(h^{[\beta(\varepsilon)]} \circ f_{\delta}\right)\left(R^{\text {pr }} \uparrow \beta(\varepsilon)\right)\left(r_{\delta}\lceil\beta(\varepsilon))\right.$. The assumption of $(\boxplus)_{15}^{b}$ for $\varepsilon^{\prime}<\varepsilon$ implies that $\zeta_{\delta}^{*} \geq \beta(\varepsilon)$ and $q_{\delta}^{*}\left\lceil\beta(\varepsilon) \leq r_{\delta}\left\lceil\beta(\varepsilon)\right.\right.$. Hence, remembering $(\boxplus)_{14}^{b}$, we also conclude that $q_{\delta}^{*}\left\lceil\beta(\varepsilon) \in \bigcap_{\alpha<\delta} \mathcal{I}_{\alpha}^{[\beta(\varepsilon)]}\right.$ and therefore $q_{\delta}^{[\beta(\varepsilon)]}=q_{\delta}^{*}\lceil\beta(\varepsilon)$. Consequently, the proof of $(\boxplus)_{15}$ is completed.

Finally, $(\boxplus)_{15}$ for $\varepsilon^{*}$ implies that Generic won the considered play, finishing the proof of the Subclaim.

To conclude the Theorem we will argue that the strategy st for Generic in $\partial_{1}^{\mathcal{S}}\left(r, N, h, \mathbb{P}_{\zeta^{*}}, R^{\text {pr }}, \bar{f}, \bar{q}^{\left[\zeta^{*}\right]}\right)$ described in the proof of Claim 4.1.5 for $\zeta=\zeta^{*}$ is also a winning strategy for Generic in $\partial_{1}^{\mathcal{S}}\left(r, N, h, \mathbb{P}_{\zeta^{*}}, R^{\mathrm{pr}}, \bar{f}, \bar{q}\right)$. Note that if $\zeta=\zeta^{*}$ and in the proof of Subclaim 4.1.5.2 we look at $\varepsilon=\varepsilon^{*}$ (i.e., $\beta(\varepsilon)=\zeta^{*}$ ), then we obtain that for relevant $\delta$ :

$(\alpha) r_{\delta}$ is an upper bound to $h \circ f_{\delta}\left(\right.$ by $(\boxplus)_{14}^{a}$ ),

( $\beta) r_{\delta} \in \bigcap_{\alpha<\delta} \mathcal{I}_{\alpha}\left(\right.$ by $\left.(\boxplus)_{14}^{b}\right)$,

$(\gamma)\left(h \circ f_{\delta}\right) R^{\mathrm{pr}} r_{\delta}\left(\right.$ by $\left.(\boxplus)_{15}^{a}\right)$.

Therefore, $q_{\delta}$ must have the same properties $(\alpha)-(\gamma)$, and by $(\otimes)_{13}^{b}$ we get $\zeta_{\delta}^{*}=\zeta^{*}$ and $q_{\delta}^{*}=q_{\delta}$. Now $(\boxplus)_{15}^{b}$ implies $q_{\delta}^{\left[\zeta^{*}\right]}=q_{\delta}$ and we see that Generic wins indeed.

Thus the condition $r$ is generic for $\bar{q}$ over $N, h, \mathbb{P}_{\zeta^{*}}, R^{\mathrm{pr}}, \bar{f}$ and this completes the proof of the Theorem.

Acknowledgements. We would like to thank the referees for their valuable comments which helped to improve the manuscript.

Both authors acknowledge support from the United States-Israel Binational Science Foundation (Grant no. 2010405). This is publication 1001 of the second author. 


\section{REFERENCES}

[1] Uri Abraham. Proper forcing. In Matthew Foreman and Akihiro Kanamori, editors, Handbook of Set Theory, volume 1, pages 333-394. Springer, 2010.

[2] Elizabeth T. Brown and Marcia J. Groszek. Uncountable superperfect forcing and minimality. Ann. Pure Appl. Logic, 144:73-82, 2006.

[3] Todd Eisworth. On iterated forcing for successors of regular cardinals. Fundamenta Mathematicae, 179:249-266, 2003.

[4] Sy-David Friedman, Radek Honzik, and Lyubomyr Zdomskyy. Fusion and large cardinal preservation. Annals of Pure and Applied Logic, 164:1247-1273, 2013.

[5] Sy-David Friedman and Lyubomyr Zdomskyy. Measurable cardinals and the cofinality of the symmetric group. Fundamenta Mathematicae, 207:101-122, 2010.

[6] Serge Grigorieff. Combinatorics on ideals and forcing. Ann. Math. Logic, 3:363-394, 1971.

[7] Stephen H. Hechler. On the existence of certain cofinal subsets of ${ }^{\omega} \omega$. In Axiomatic set theory, volume XIII of Proceedings of Symposia in Pure Mathematics, pages 155-173. American Mathematical Society, Providence, R.I., 1974.

[8] Tapani Hyttinen and Mika Rautila. The canary tree revisited. The Journal of Symbolic Logic, 66:1677-1694, 2001.

[9] Thomas Jech. Set theory. Springer Monographs in Mathematics. Springer-Verlag, Berlin, 2003. The third millennium edition, revised and expanded.

[10] Akihiro Kanamori. Perfect-set forcing for uncountable cardinals. Annals of Mathematical Logic, 19:97-114, 1980.

[11] Richard Laver. On the consistency of Borel's conjecture. Acta Math., 137:151-169, 1976.

[12] Arnold W. Miller. Some properties of measure and category. Transactions of the American Mathematical Society, 266:93-114, 1981.

[13] Arnold W. Miller. Rational perfect set forcing. In Donald A. Martin James E. Baumgartner and Saharon Shelah, editors, Axiomatic set theory (Boulder, Colo., 1983), volume 31 of Contemporary Mathematics, pages 143-159. American Mathematical Society, Providence, RI, 1984.

[14] Andrzej Roslanowski and Saharon Shelah. Iteration of $\lambda$-complete forcing notions not collapsing $\lambda^{+}$. International Journal of Mathematics and Mathematical Sciences, 28:63-82, 2001. arxiv:math.LO/9906024

[15] Andrzej Roslanowski and Saharon Shelah. Reasonably complete forcing notions. Quaderni di Matematica, 17:195-239, 2006. arxiv:math.LO/0508272

[16] Andrzej Roslanowski and Saharon Shelah. Sheva-Sheva-Sheva: Large Creatures. Israel Journal of Mathematics, 159:109-174, 2007. arxiv:math.LO/0210205

[17] Andrzej Roslanowski and Saharon Shelah. Lords of the iteration. In Set Theory and Its Applications, volume 533 of Contemporary Mathematics (CONM), pages 287-330. Amer. Math. Soc., 2011. arxiv:math.LO/0611131

[18] Andrzej Roslanowski and Saharon Shelah. Reasonable ultrafilters, again. Notre Dame Journal of Formal Logic, 52:113-147, 2011. arxiv:math.LO/0605067

[19] Andrzej Roslanowski and Saharon Shelah. More about $\lambda$-support iterations of $(<\lambda)$-complete forcing notions. Archive for Mathematical Logic, 52:603-629, 2013. arXiv:1105.6049.

[20] Saharon Shelah. Independence results. The Journal of Symbolic Logic, 45:563-573, 1980.

[21] Saharon Shelah. Proper forcing, volume 940 of Lecture Notes in Mathematics. SpringerVerlag, Berlin-New York, xxix+496 pp, 1982.

[22] Saharon Shelah. Proper and improper forcing. Perspectives in Mathematical Logic. Springer, 1998.

[23] Saharon Shelah. Not collapsing cardinals $\leq \kappa$ in $(<\kappa)$-support iterations. Israel Journal of Mathematics, 136:29-115, 2003. arxiv:math.LO/9707225

[24] Saharon Shelah. Successor of singulars: combinatorics and not collapsing cardinals $\leq \kappa$ in $(<\kappa)$-support iterations. Israel Journal of Mathematics, 134:127-155, 2003. arxiv:math.LO/9808140 
Department of Mathematics, University of Nebraska at Omaha, Omaha, Ne 681820243, USA

E-mail address: roslanow@member.ams.org

Einstein Institute of Mathematics, Edmond J. Safra Campus, Givat Ram, The Hebrew University of Jerusalem, Jerusalem, 91904, Israel, and, Department of Mathematics, Rutgers University, Hill Center - Busch Campus, 110 Frelinghuysen Road, Piscataway, NJ 08854-8019, USA

E-mail address: shelah@math.huji.ac.il

URL: http://shelah.logic.at 